\title{
Blue Phosphorescence with High Quantum Efficiency Engaging Trifluoromethylsulfonyl Group to Iridium Phenyl Pyridine Complexes
}

\author{
Jin-Hyoung Kim, So-Yoen Kim, Seol Jang, Seungjun Yi, Dae Won Cho, *Ho-Jin Son, * and Sang Ook Kang*
}

Department of Advanced Materials Chemistry, Korea University, Sejong 30019, Korea.

Table of contents

\begin{tabular}{|c|c|c|}
\hline & Titles & pages \\
\hline & General information. & $\mathrm{S} 2$ \\
\hline Figure $\mathbf{S 1}$ & ${ }^{1} \mathrm{H}-\mathrm{NMR}$ spectrum of $\mathbf{L 1}$. & S6 \\
\hline Figure S2 & ${ }^{1} \mathrm{H}-\mathrm{NMR}$ spectrum of $\mathbf{L} \mathbf{2}$. & S6 \\
\hline Figure S3 & ${ }^{1} \mathrm{H}-\mathrm{NMR}$ spectrum of $\mathbf{L} \mathbf{3}$ & S7 \\
\hline Figure S4 & ${ }^{1} \mathrm{H}-\mathrm{NMR}$ spectrum of $\operatorname{Ir}\left(\mathbf{p p y S C F}_{3}\right)_{2}$ (acac). & S7 \\
\hline Figure S5 & ${ }^{1} \mathrm{H}-\mathrm{NMR}$ spectrum of $\operatorname{Ir}(\mathbf{p p y S C F})_{2}$ (pic). & S8 \\
\hline Figure S6 & ${ }^{1} \mathrm{H}-\mathrm{NMR}$ spectrum of $\left.\operatorname{Ir}(\mathbf{p p y S C F})_{3}\right)_{2}$ (bor). & S8 \\
\hline Figure S7 & ${ }^{1} \mathrm{H}-\mathrm{NMR}$ spectrum of $\operatorname{Ir}\left(\mathbf{p p y S C F}_{3}\right)_{3}$. & S9 \\
\hline Figure S8 & ${ }^{1} \mathrm{H}-\mathrm{NMR}$ spectrum of $\mathbf{I r}(\mathbf{p p y S F})_{3}$ & S9 \\
\hline Figure S9 & ESI-Mass spectrum of $\left.\operatorname{Ir}(\mathbf{p p y S C F})_{3}\right)_{2}(\mathbf{a c a c})$. & S10 \\
\hline Figure S10 & ESI-Mass spectrum of $\operatorname{Ir}(\mathbf{p p y S C F})_{2}(\mathbf{p i c})$ & $\mathrm{S} 10$ \\
\hline Figure S11 & ESI-Mass spectrum of $\left.\operatorname{Ir}(\mathbf{p p y S C F})_{\mathbf{3}}\right)_{2}$ (bor). & S11 \\
\hline Figure S12 & ESI-Mass spectrum of $\operatorname{Ir}\left(\mathbf{p p y S C F}_{3}\right)_{3}$ & S11 \\
\hline Figure S13 & ESI-Mass spectrum of $\operatorname{Ir}(\mathbf{p p y S F})_{3}$ & S12 \\
\hline Figure S14 & ORTEP drawing of $\operatorname{Ir}\left(\mathbf{p p y S C F}_{3}\right)_{\mathbf{2}}(\mathbf{a c a c})$ with $30 \%$ probability for the thermal ellipsoids. & S13 \\
\hline Figure S15 & ORTEP drawing of $\operatorname{Ir}\left(\mathbf{p p y y S C F}_{3}\right)_{2}(\mathbf{p i c})$ with $30 \%$ probability for the thermal ellipsoids. & S13 \\
\hline Figure S16 & ORTEP drawing of $\mathbf{I r}\left(\mathbf{p p y S C F}_{3}\right)_{2}(\mathbf{b o r})$ with $30 \%$ probability for the thermal ellipsoids. & S14 \\
\hline Table S1 & $\begin{array}{l}\text { The frontier molecular orbital contribution on main ligand, ancillary ligand, and iridium } \\
\text { metal (Ir) in } \operatorname{Ir}(\mathrm{III}) \text { complexes. }\end{array}$ & S14 \\
\hline Figure S17 & Structures of FIr(acac), FIr(pic), and FIr(bor) (FIr6). & S15 \\
\hline Figure S18 & $\begin{array}{l}\text { Experimental absorption spectra for Ir complexes, and theoretically calculated oscillator } \\
\text { strengths obtained by the TD-DFT calculation. }\end{array}$ & S16 \\
\hline Figure S19 & Spatial distributions for the frontier orbitals of Ir complexes. & S17 \\
\hline Table S2 & Energy levels and contributions of the lower lying transitions of $\operatorname{Ir}\left(\operatorname{ppySCF}_{3}\right)_{2}(\mathbf{a c a c})$. & S18 \\
\hline Table S3 & Energy levels and contributions of the lower lying transitions of $\operatorname{Ir}\left(\operatorname{ppySCF}_{3}\right)_{2}(\mathbf{p i c})$. & S19 \\
\hline Table S4 & Energy levels and contributions of the lower lying transitions of $\operatorname{Ir}(\mathbf{p p y S C F})_{2}(\mathbf{b o r})$. & S20 \\
\hline Table S5 & Energy levels and contributions of the lower lying transitions of $\operatorname{Ir}\left(\mathbf{p p y S C F} \mathbf{F}_{3}\right)_{3}$. & $\mathrm{S} 21$ \\
\hline Table S6 & Energy levels and contributions of the lower lying transitions of $\operatorname{Ir}(\mathbf{p p y S F})_{3}$ & S22 \\
\hline Table S7 & $\begin{array}{l}\text { Crystal data and structure refinement for } \operatorname{Ir}\left(\operatorname{ppySCF}_{3}\right)_{2}(\operatorname{acac}), \operatorname{Ir}\left(\operatorname{ppySCF}_{3}\right)_{2}(\mathbf{p i c}) \text {, and } \\
\operatorname{Ir}\left(\operatorname{ppy} \mathbf{S C F}_{3}\right)_{2}(\text { bor }) \text {. }\end{array}$ & S23 \\
\hline Table S8 & Bond lengths $[\AA]$ and angles $\left[{ }^{\circ}\right]$ for $\operatorname{Ir}(\mathbf{p p y S C F})_{2}(\mathbf{a c a c})$ & S24 \\
\hline Table S9 & Bond lengths $[\AA]$ and angles $\left[{ }^{\circ}\right]$ for $\operatorname{Ir}(\mathbf{p p y S C F})_{2}(\mathbf{p i c})$. & S25 \\
\hline \multirow[t]{2}{*}{ Table S10 } & Bond lengths $[\AA]$ and angles $\left[{ }^{\circ}\right]$ for $\operatorname{Ir}(\mathbf{p p y S C F})_{3}$ (bor). & S27 \\
\hline & References & S29 \\
\hline
\end{tabular}


General Information. All the synthetic procedures were performed in a dry $\mathrm{N}_{2}$ atmosphere. All the solvents used were distilled over sodium-benzophenone under nitrogen prior to use. Glassware, syringes, magnetic stirring bars, and needles were dried in a convection oven for over $4 \mathrm{~h}$. Reactions were monitored by thin-layer chromatography (TLC; Merck Co.). The spots developed onto TLC were identified under UV light at (254 or 365) nm. Column chromatography was performed on silica gel $60 \mathrm{G}$ (particle size (5-40) $\mu \mathrm{m}$; Merck Co.). The synthesized compounds were characterized by ${ }^{1} \mathrm{H}$ NMR and high-resolution mass spectrometry (HR-MS). The ${ }^{1} \mathrm{H}$ NMR spectra were recorded on a Varian Mercury 300 spectrometer (operating at (300.1 and 75.4) MHz) and Bruker Ascend 400 spectrometer in KBSI Ochang Center (operating at (400.1 and 100.6) MHz). HR-MS analysis was performed on an LC/MS/MSn $(n=10)$ spectrometer (Thermo Fisher Scientific, LCQ Fleet Hyperbolic Ion Trap MS/MSn Spectrometer). Iridium(III) chloride hydrate was purchased from SigmaAldrich, and used without further purification. 2-(3-sulfonylfluorophenyl)pyridine (ppySF) S1 and 2-(tributylstannyl)pyridine ${ }^{\mathrm{S} 2}$ were synthesized using previously reported methods.

Spectroscopic Measurements. The steady-state absorption and photoluminescence spectra were measured by using logies Cary 5000 and a Varian Cary Eclipse, respectively. The phosphorescence spectra at $77 \mathrm{~K}$ were measured using an intensified charge-coupled device (ICCD, Andor, iStar). The samples were excited by nanosecond laser pulses of the third harmonic generation (355 nm, FWHM of $4.5 \mathrm{~ns}$ ) from a Q-switched Nd:YAG laser (Continuum, Surelite II-10). Phosphorescence lifetimes were measured using a digital oscilloscope (Tektronix, TDS-784D) equipped with a fast photomultiplier tube (Zolix Instruments Co., CR 131).

Cyclic Voltammograms. The cyclic voltammetry (CV) experiments were carried out in distilled, degassed $\mathrm{CH}_{3} \mathrm{CN}$ containing $1 \mathrm{mM}$ Ir complexes and $0.1 \mathrm{M}$ tetrabutylammonium 
perchlorate (TBAP) at RT using an electrochemical analyzer (CH Instruments, CHI660e). A platinum disk (dia. $1.6 \mathrm{~mm}$ ), platinum wire, and $\mathrm{Ag} / \mathrm{AgNO}_{3}(0.1 \mathrm{M})$ were used as the working, counter, and reference electrodes, respectively. All potential values were calibrated vs. the ferrocene/ferrocenium $\left(\mathrm{Fc} \mid \mathrm{Fc}^{+}\right)$redox couple.

DFT Calculation. The DFT calculations were performed using the Gaussian 09 program package. ${ }^{\mathrm{S} 3}$ The ground-state geometry was fully optimized at the DFT level using the B3LYP (UB3LYP for triplet calculations) method. ${ }^{\mathrm{S} 4-\mathrm{S} 6} \mathrm{The} 6-31 \mathrm{G}(\mathrm{d}, \mathrm{p})$ and LANL2DZ basis sets were applied for non-metal atoms and iridium, respectively. The isodensity plots (contour $=0.03$ a.u.) of the frontier orbitals were visualized by the Chem3D Ultra or GaussView 6 programs. The time-dependent DFT (TD-DFT) calculation was performed to evaluate the vertical transition energies and oscillator strength for the singlet and triplet transitions.

PL Quantum Yield Measurement. The $\Phi_{\mathrm{P}}$ values of Ir-complexes in anaerobic $\mathrm{CH}_{2} \mathrm{Cl}_{2}$ solution were determined by a relative method using the well-established quantum yield standard, 9,10-diphenylanthracene $\left(\Phi_{\mathrm{f}}=1\right)$.

Crystal Structure Determination. Each light-yellow crystal of $\operatorname{Ir(ppySCF} 3)_{2}$ (acac), $\operatorname{Ir}\left(\operatorname{ppySCF}_{3}\right)_{2}$ (pic), and $\operatorname{Ir}\left(\operatorname{ppySCF}_{3}\right)_{2}($ bor) was obtained by slow evaporation of their dissolution in $\mathrm{CH}_{2} \mathrm{Cl}_{2}$ was sealed in glass capillaries under argon, and mounted on the diffractometer. The preliminary examination and data collection were performed on a Bruker SMART CCD X-ray diffractometer equipped with a sealed-tube X-ray source $(50 \mathrm{kV} \times 30$ mA) using graphite-monochromated Mo $K \alpha$ radiation $(\lambda=0.71073 \AA)$. The preliminary unit cell constants were determined using a set of 45 narrow-frame $\left(0.3^{\circ}\right.$ in $\left.\omega\right)$ scans. The doublepass method of scanning was used to exclude noise. The collected frames were integrated using an orientation matrix determined from the narrow-frame scans. The SMART software package was used for data collection, and SAINT was used for frame integration. ${ }^{\mathrm{S} 7}$ The final 
cell constants were determined through global refinement of the $x y z$ centroids of the reflections harvested from the entire data set. Structure solution and refinement were performed using the SHELXTL-PLUS software package. ${ }^{\mathrm{S} 8}$ Crystallographic data was deposited with the Cambridge Crystallographic Data Centre as supplementary publications (CCDC-1919544 (Ir(ppySCF 3$)_{2}$ (acac)), CCDC-1919505 (Ir(ppySCF 3$)_{2}$ (pic)), and CCDC1919522 (Ir(ppySCF $)_{2}$ (bor))). Additional crystallographic data are available in the Tables S7-S10 of the Supporting Information (SI).

\section{Device fabrication and characterization}

The OLED devices were fabricated on glass substrates, which were pre-coated with a $150 \mathrm{~nm}$ indium tin oxide (ITO) layer having a sheet resistance of $10 \Omega$ /square. The ITO glass was precleaned sequentially with acetone (30 min), $N$-methyl-2-pyrrolidone (NMP) (30 min), deionized water, and isopropyl alcohol (IPA) (30 min), in an ultrasonic bath. The cleaned ITO glass was used immediately to fabricate the OLEDs. The ITO substrates were treated with oxygen plasma, before usage. The poly(3,4-ethylenedioxythiophene):poly(styrene sulfonate) (PEDOT:PSS) (hole injection layer, HIL) was spin-coated at 4,000 rpm for $60 \mathrm{~s}$ on the precleaned ITO glass substrates to produce a $\sim 40 \mathrm{~nm}$ thick hole-injecting/transporting layer (HTL) layer, and annealed at $120{ }^{\circ} \mathrm{C}$ for $30 \mathrm{~min}$ to remove the residual water. A chlorobenzene solution of $10 \mathrm{mg} / \mathrm{mL}$ tris(4-carbazoyl-9-ylphenyl)amine (TCTA) was doped with $10 \% \mathrm{w} / \mathrm{w}$ of Ir dopants. The mixed solution was filtered with a $0.5 \mu \mathrm{m}$ pore filter, and spin-coated at 3,000 rpm for $1 \mathrm{~min}$ on the top of the PEDOT:PSS layer, and baked for $30 \mathrm{~min}$ at $120^{\circ} \mathrm{C}$. The coated film was shadow masked to give four identical devices of area $(2 \mathrm{~mm} \times 2 \mathrm{~mm})$; the substrate was then transferred into the evaporation chamber through a nitrogen glovebox, where 1,3,5-tris(3-pyridyl-3-phenyl)benzene (TmPyPB) (35 nm) was evaporated as electron transporting layer (ETL) at a rate of $\sim 1 \AA /$ s under vacuum at a pressure of ca. $3 \times 10^{-7}$ Torr, 
followed by $1 \mathrm{~nm}$ 8-quinolinolato lithium (Liq) and a $150 \mathrm{~nm}$ capping layer of aluminum under the same condition. The resulting device configuration of all devices is as follows: ITO/PEDOT:PSS (40 nm)/TCTA (90\%):Ir dopant (10\%)/TmPyPB (35 nm)/Liq $(1 \mathrm{~nm}) / \mathrm{Al}$ $(150 \mathrm{~nm})$. All the fabricated devices were encapsulated in $\mathrm{N}_{2}$ glove box, before taking measurements. The current-voltage characteristics of OLEDs were analyzed using a source measurement unit (Keithley 2635B). The electroluminescence spectra, luminance, and CIE coordinates were measured by spectroradiometry (Konica Minolta CS-2000). Assuming Lambertian emission, we calculated the external quantum efficiency (EQE) from the luminance, current density, and electroluminescence spectrum.

Synthesis of 2-(3-(fluorosulfonyl)phenyl)pyridine (ppySF). Under an $\mathrm{N}_{2}$ atmosphere, $\mathrm{Pd}\left(\mathrm{PPh}_{3}\right)_{4}(0.48 \mathrm{~g}, 5 \mathrm{~mol} . \%)$ is added to a dried, degassed toluene $(40 \mathrm{~mL})$ containing 3 bromobenzene-1-sulfonyl fluoride (2.00 g, $8.37 \mathrm{mmol})$ and 2-(tri-butylstannyl)pyridine (3.08 $\mathrm{g}, 8.37 \mathrm{mmol})$. The reaction mixture is refluxed at $110{ }^{\circ} \mathrm{C}$ for $16 \mathrm{~h}$. After cooling to $\mathrm{RT}$, the solvent is removed under reduced pressure. The dark residue is purified by silica gel chromatography using ethylacetate $/ n$-hexane as eluent, to give ppySF. Yield $72 \%(1.43 \mathrm{~g})$. ${ }^{1} \mathrm{H}$ NMR (300.1 MHz, $\left.\mathrm{CDCl}_{3}\right): \delta 8.72(\mathrm{~d}, J=4.8 \mathrm{~Hz}, 1 \mathrm{H}), 8.65(\mathrm{~s}, 1 \mathrm{H}), 8.41(\mathrm{~d}, J=7.8 \mathrm{~Hz}$, 1H), $8.03(\mathrm{~d}, J=7.5 \mathrm{~Hz}, 1 \mathrm{H}),(7.85-7.70)(\mathrm{m}, 3 \mathrm{H}), 7.33(\mathrm{t}, J=6.3 \mathrm{~Hz}, 1 \mathrm{H})$. ESI-MS $(\mathrm{m} / \mathrm{z})$ : calcd. for $\mathrm{C}_{11} \mathrm{H}_{8} \mathrm{FNO}_{2} \mathrm{~S}: 237.0260$, found $[\mathrm{M}+\mathrm{H}]^{+}: 238.2524$. 


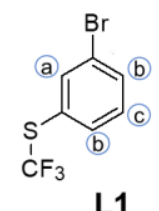

L1

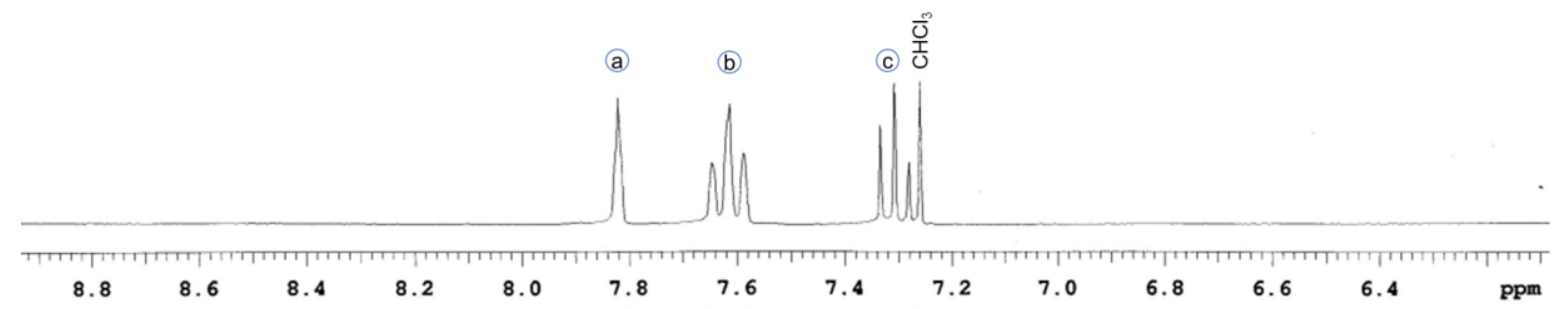

Figure S1. ${ }^{1} \mathrm{H}-\mathrm{NMR}$ spectrum of $\mathbf{L 1}$ in $\mathrm{CDCl}_{3}$.

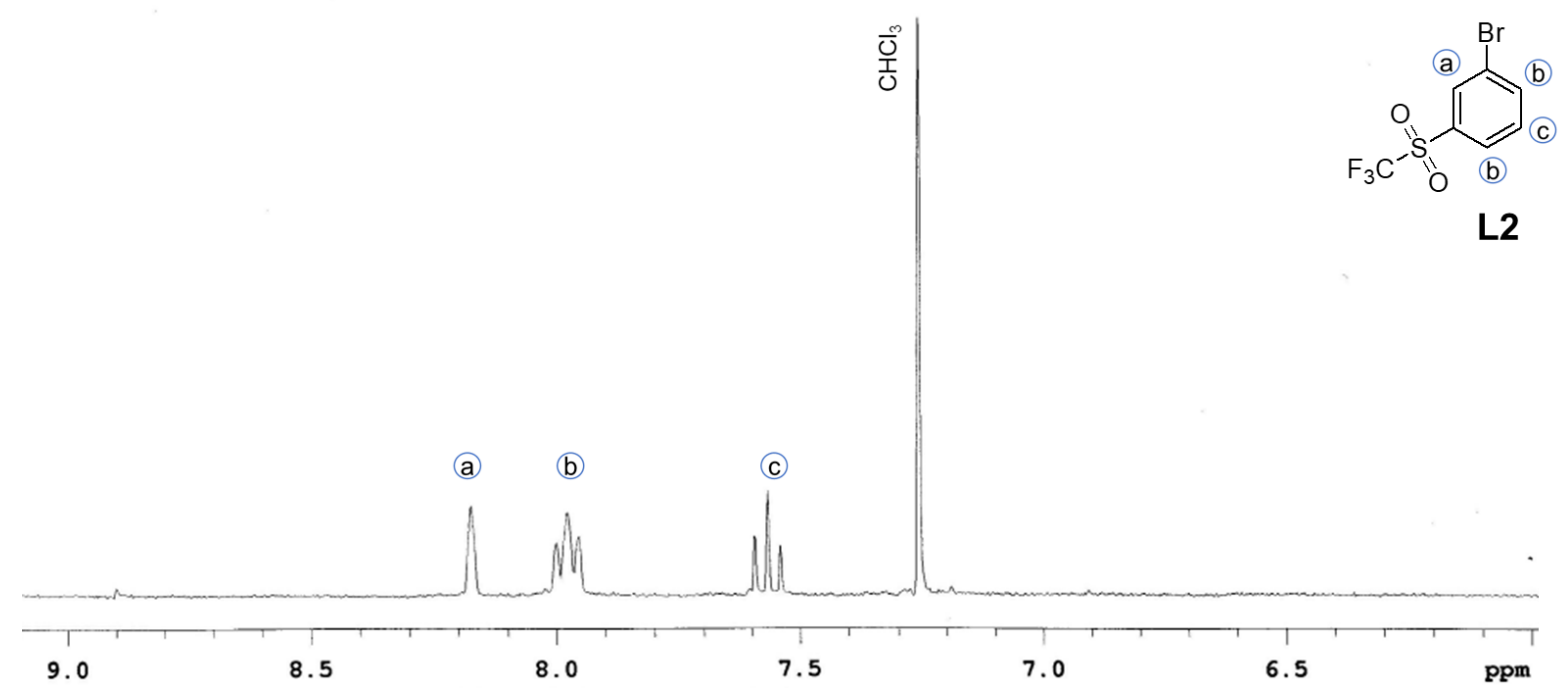

Figure S2. ${ }^{1} \mathrm{H}-\mathrm{NMR}$ spectrum of $\mathbf{L} 2$ in $\mathrm{CDCl}_{3}$. 


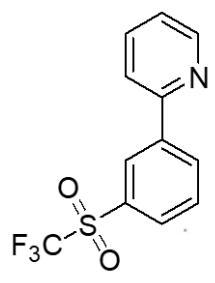

L3

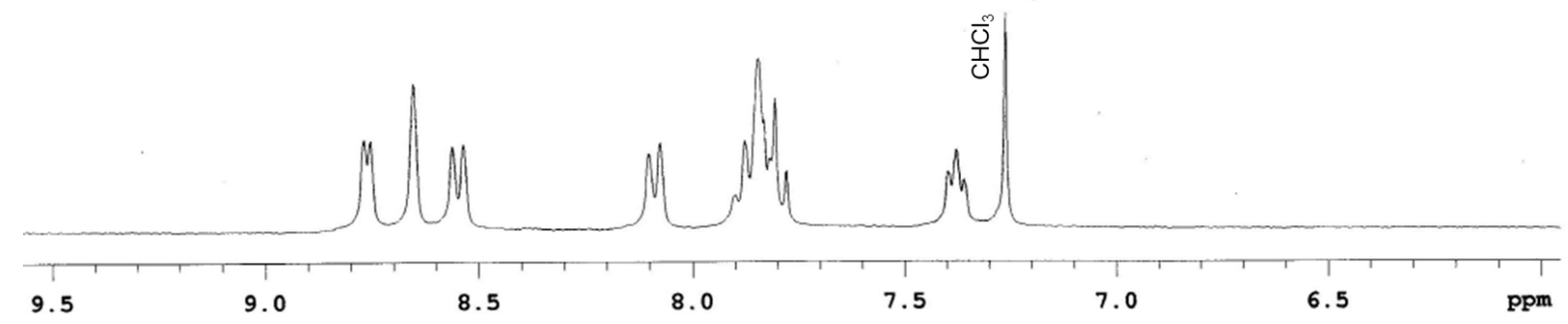

Figure S3. ${ }^{1} \mathrm{H}-\mathrm{NMR}$ spectrum of $\mathbf{L} \mathbf{3}$ in $\mathrm{CDCl}_{3}$.

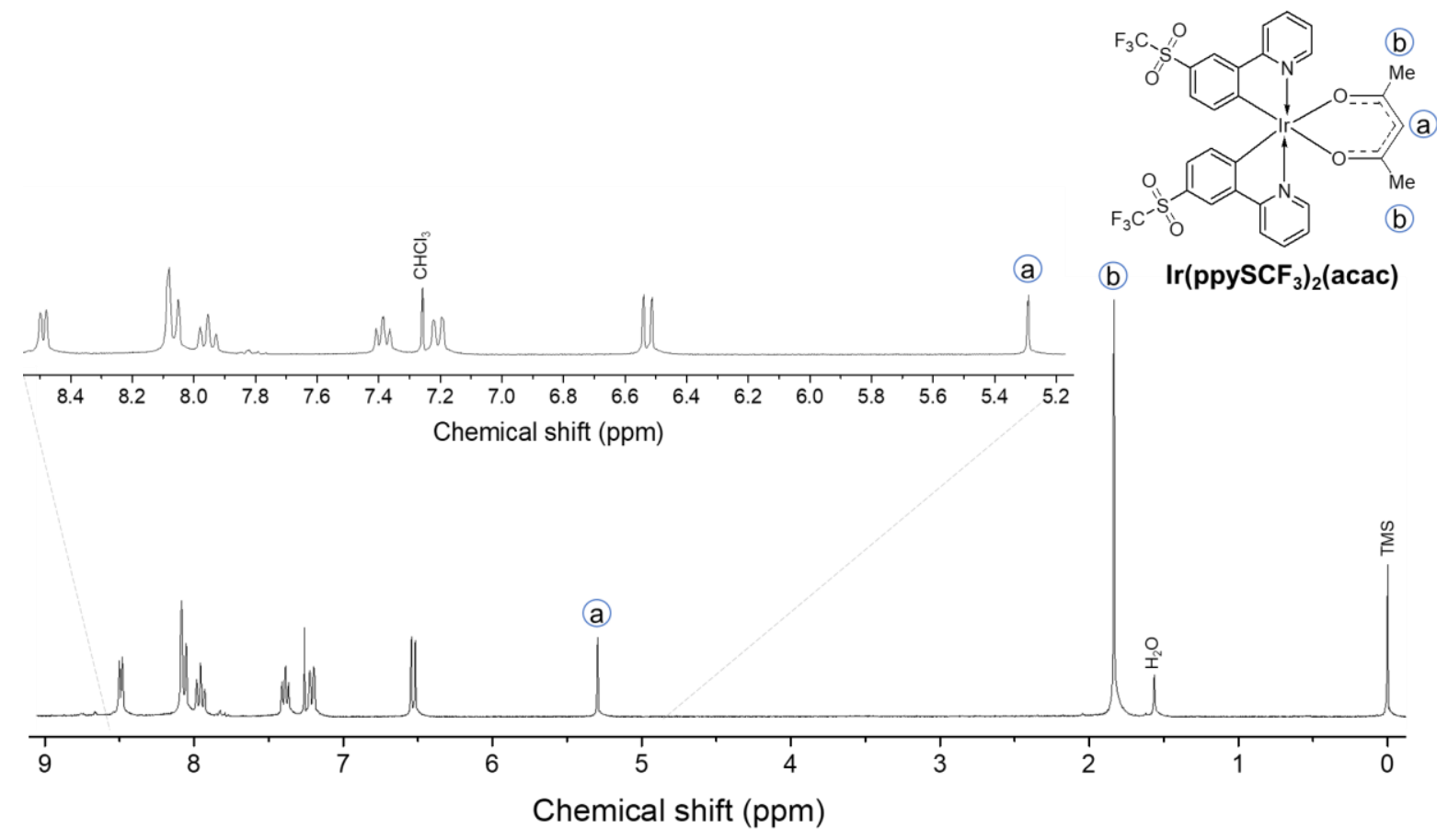

Figure S4. ${ }^{1} \mathrm{H}-\mathrm{NMR}$ spectrum of $\operatorname{Ir}\left(\mathbf{p p y S C F}_{3}\right)_{2}(\mathbf{a c a c})$ in $\mathrm{CDCl}_{3}$. 


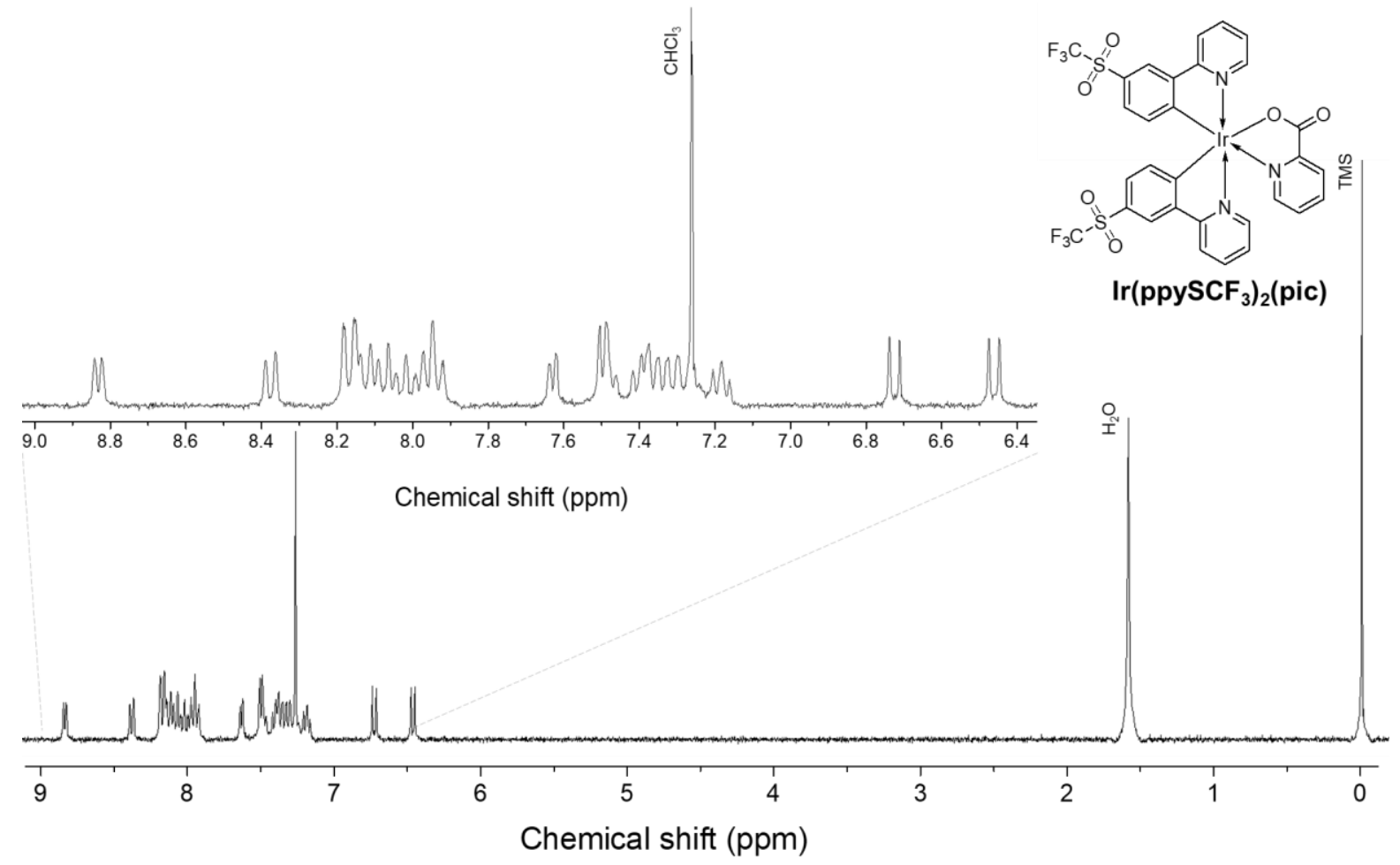

Figure S5. ${ }^{1} \mathrm{H}-\mathrm{NMR}$ spectrum of $\operatorname{Ir}\left(\mathbf{p p y S C F}_{3}\right)_{2}(\mathbf{p i c})$ in $\mathrm{CDCl}_{3}$.

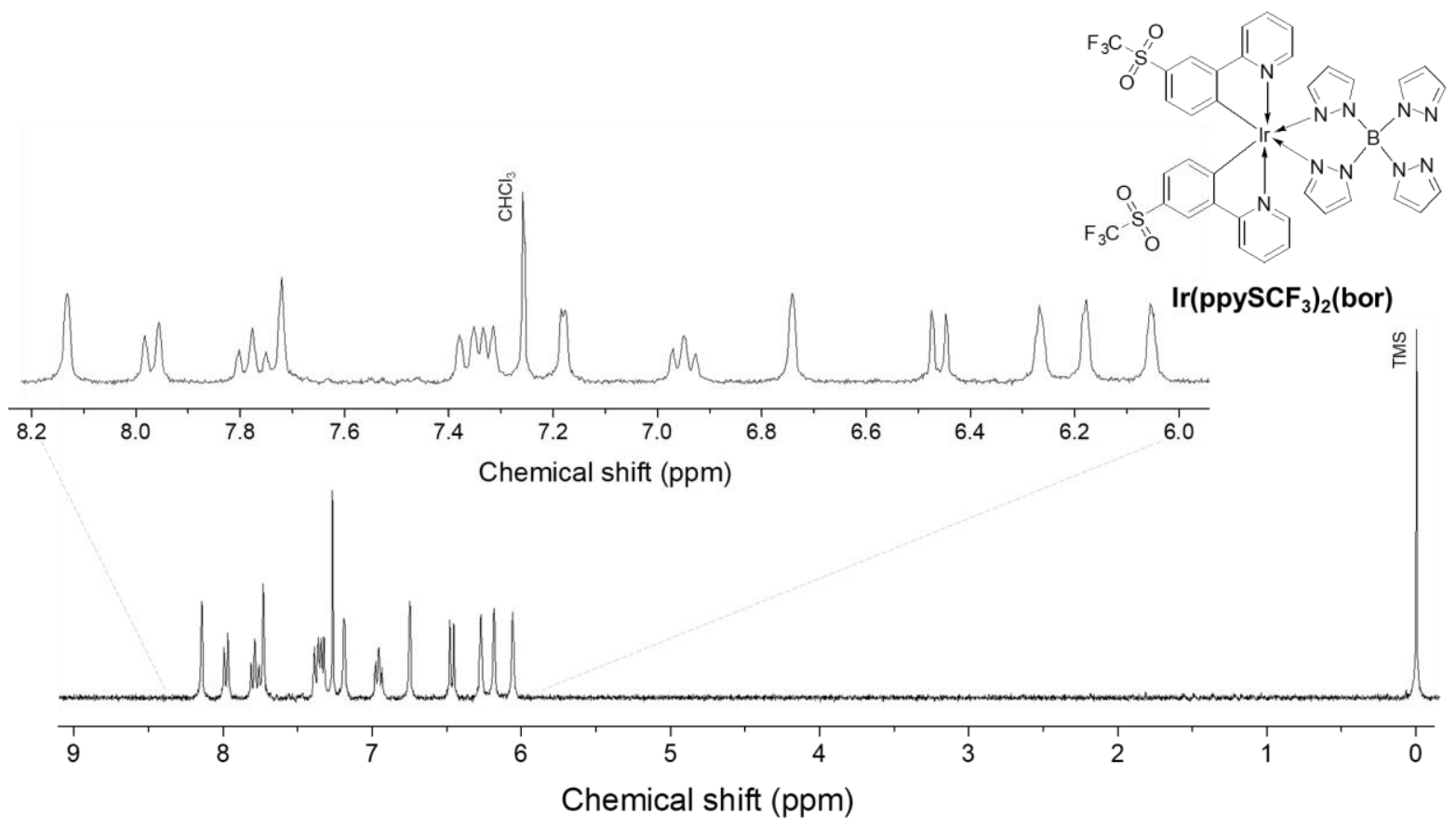

Figure S6. ${ }^{1} \mathrm{H}-\mathrm{NMR}$ spectrum of $\operatorname{Ir}(\mathbf{p p y S C F})_{2}$ (bor) in $\mathrm{CDCl}_{3}$. 


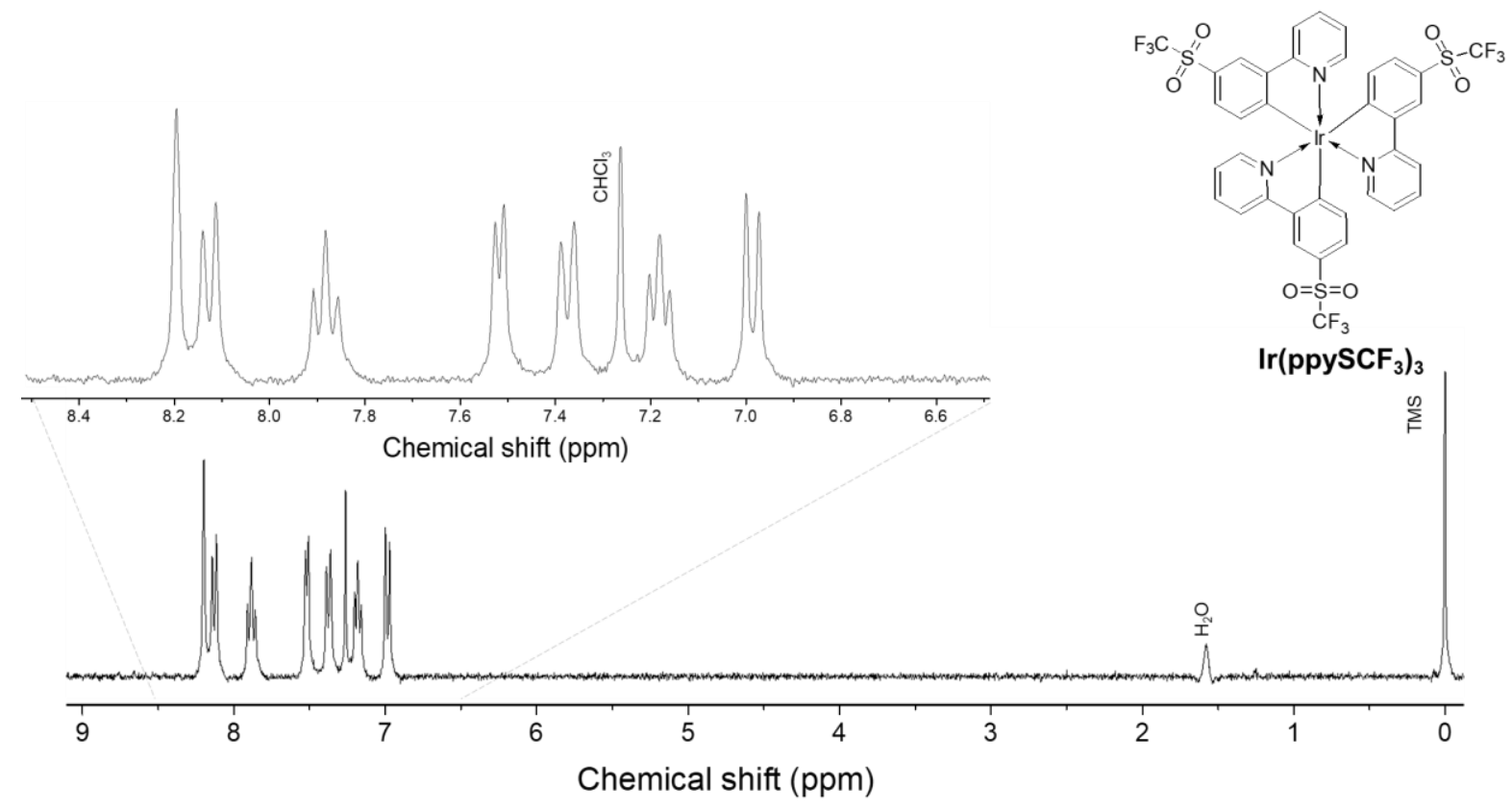

Figure S7. ${ }^{1} \mathrm{H}-\mathrm{NMR}$ spectrum of $\operatorname{Ir}(\mathbf{p p y S C F})_{3}$ in $\mathrm{CDCl}_{3}$.

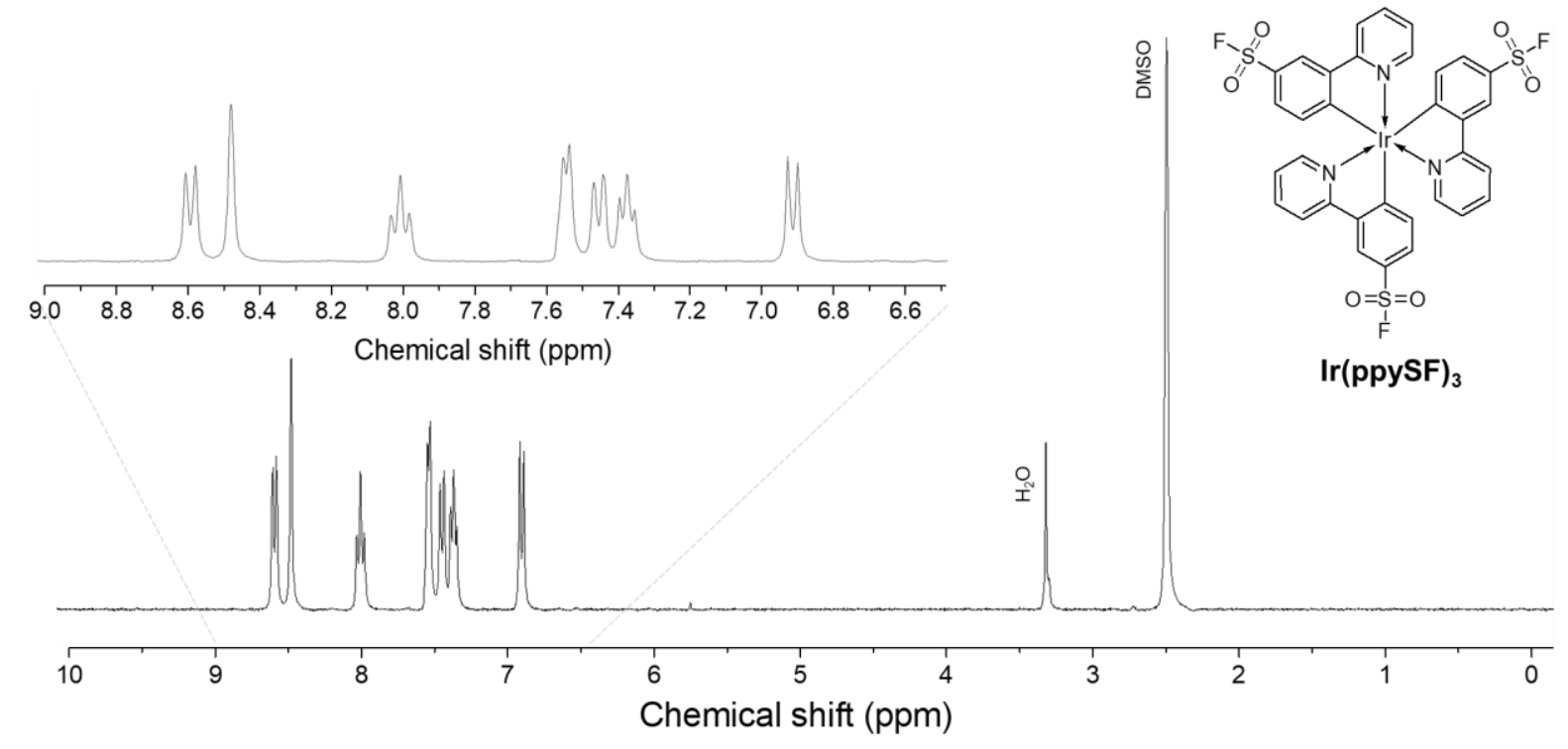

Figure S8. ${ }^{1} \mathrm{H}-\mathrm{NMR}$ spectrum of $\operatorname{Ir}(\mathbf{p p y S F})_{3}$ in $\mathrm{DMSO}-d_{6}$. 


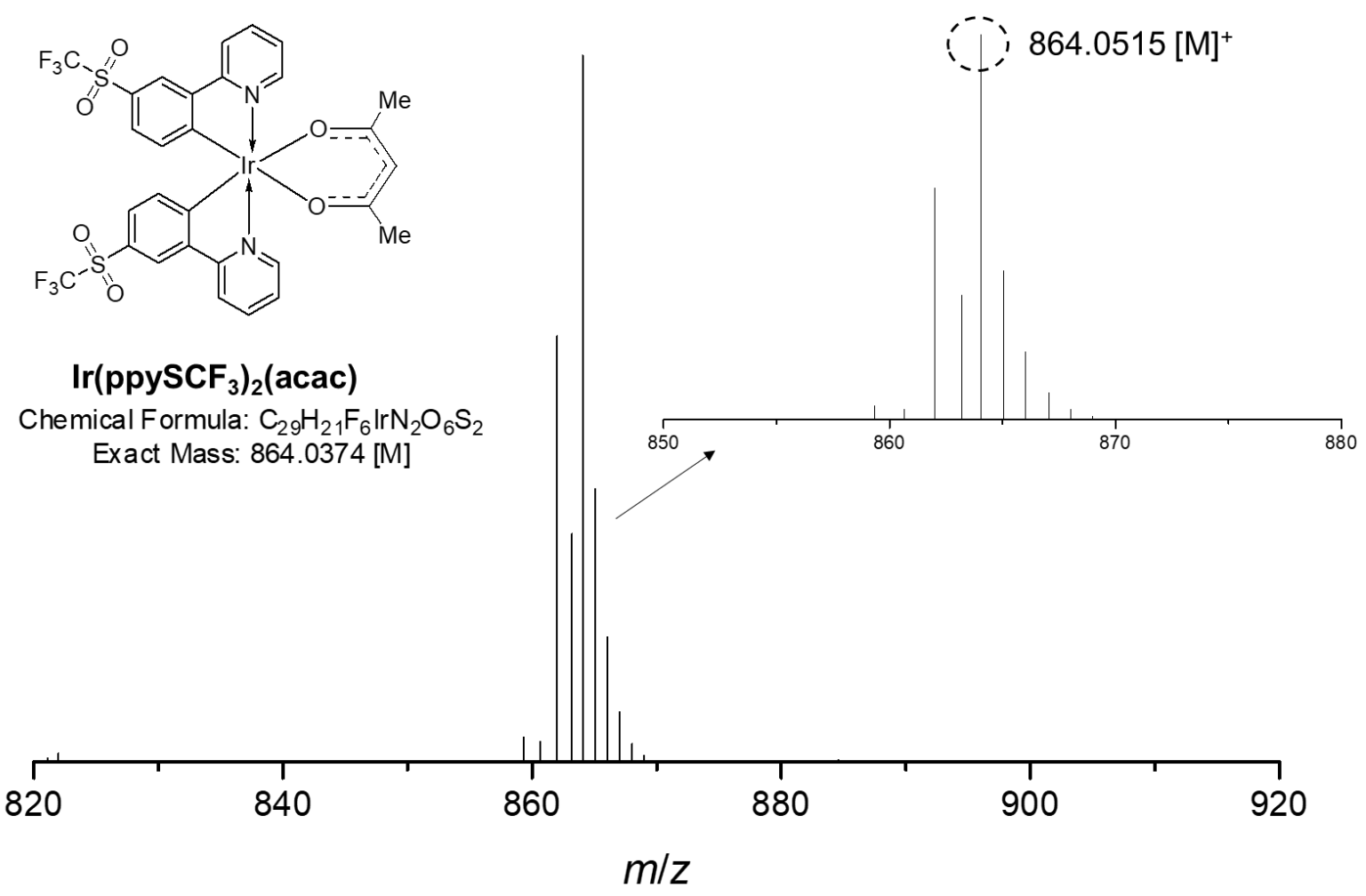

Figure S9. ESI-Mass spectra of $\operatorname{Ir}\left(\text { ppySCF}_{3}\right)_{2}$ (acac).

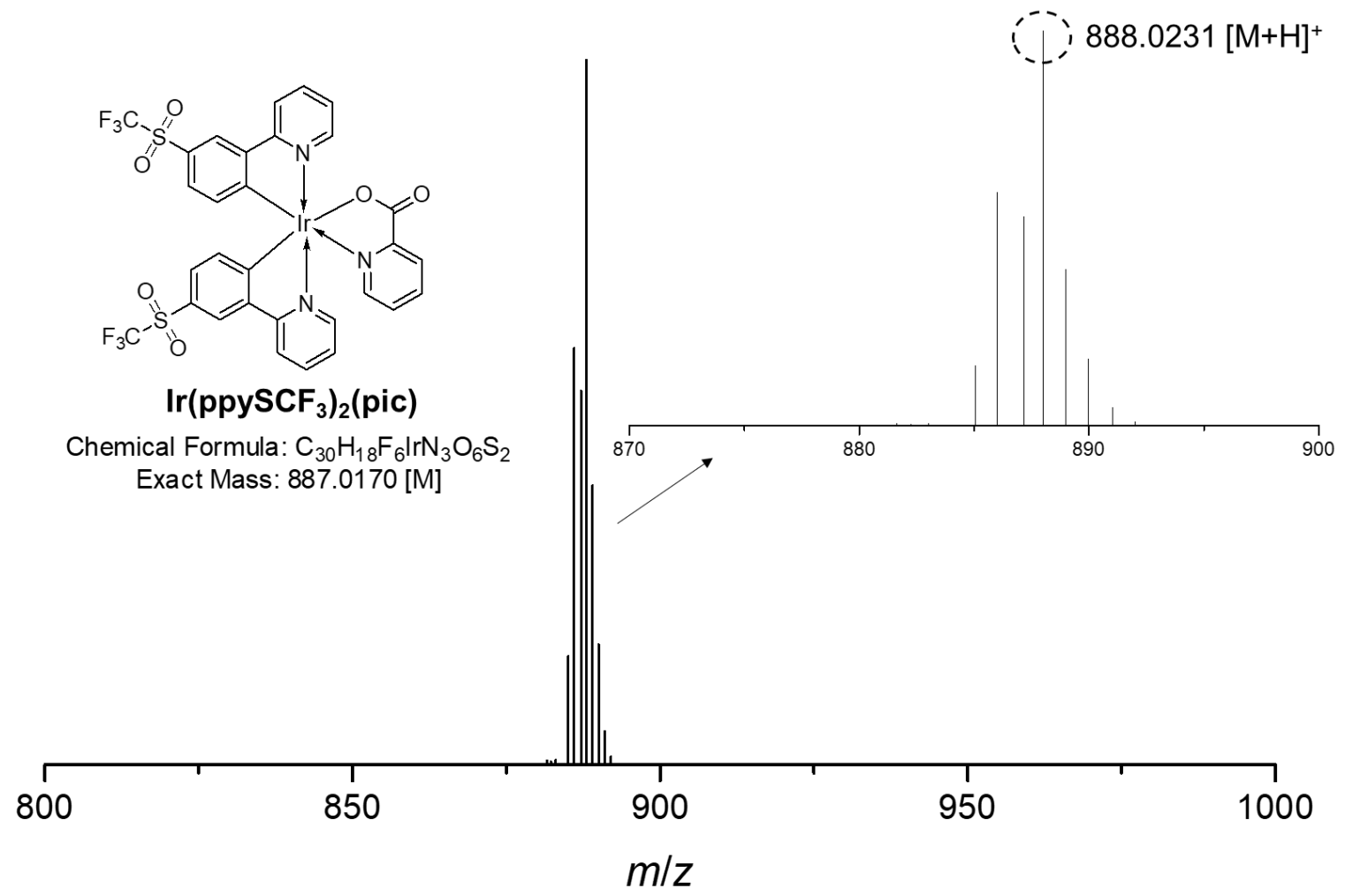

Figure S10. ESI-Mass spectra of $\operatorname{Ir}\left(\text { ppySCF}_{3}\right)_{2}$ (pic). 


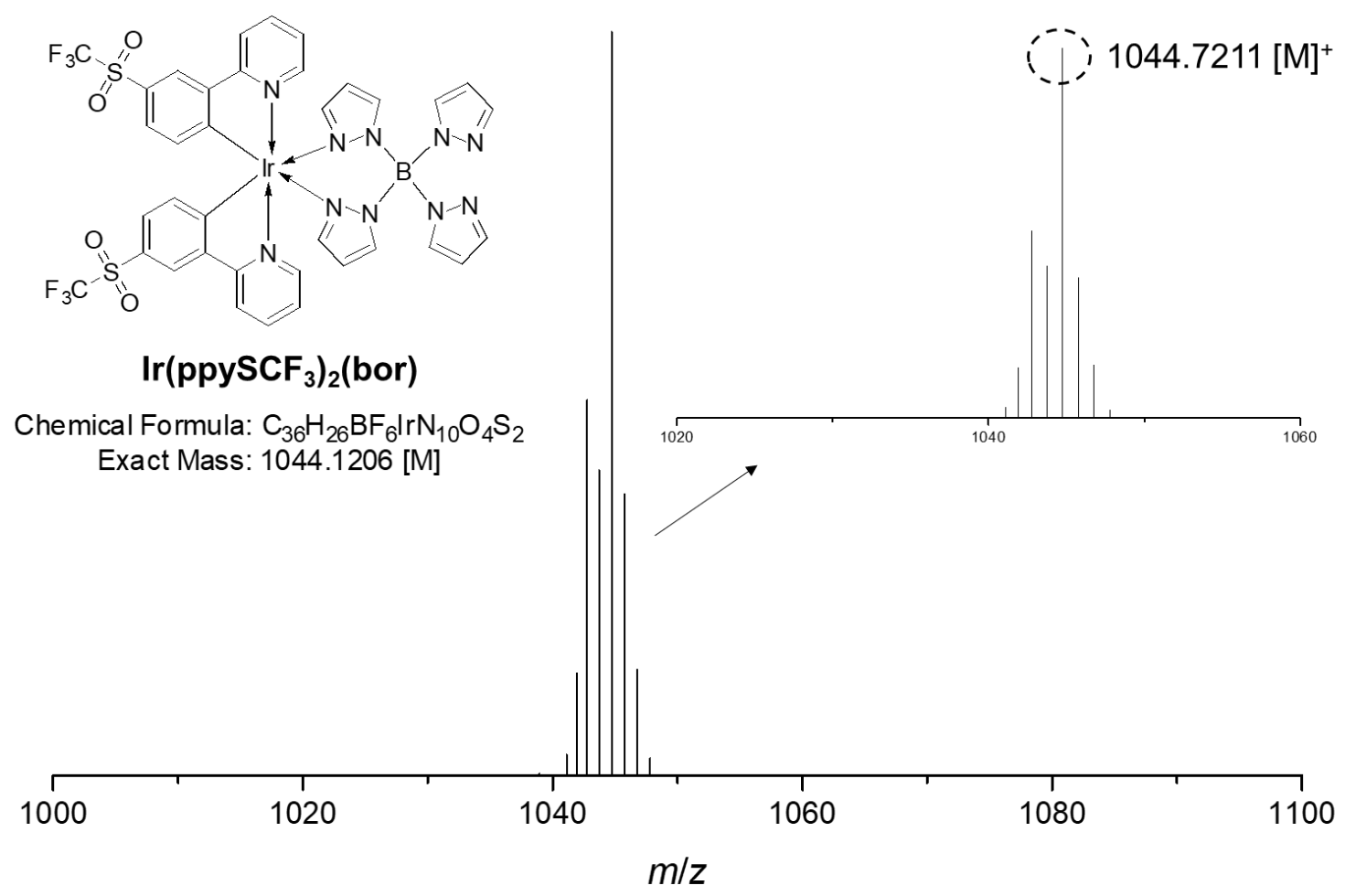

Figure S11. ESI-Mass spectra of $\operatorname{Ir}\left(\operatorname{ppySCF}_{3}\right)_{2}$ (bor).

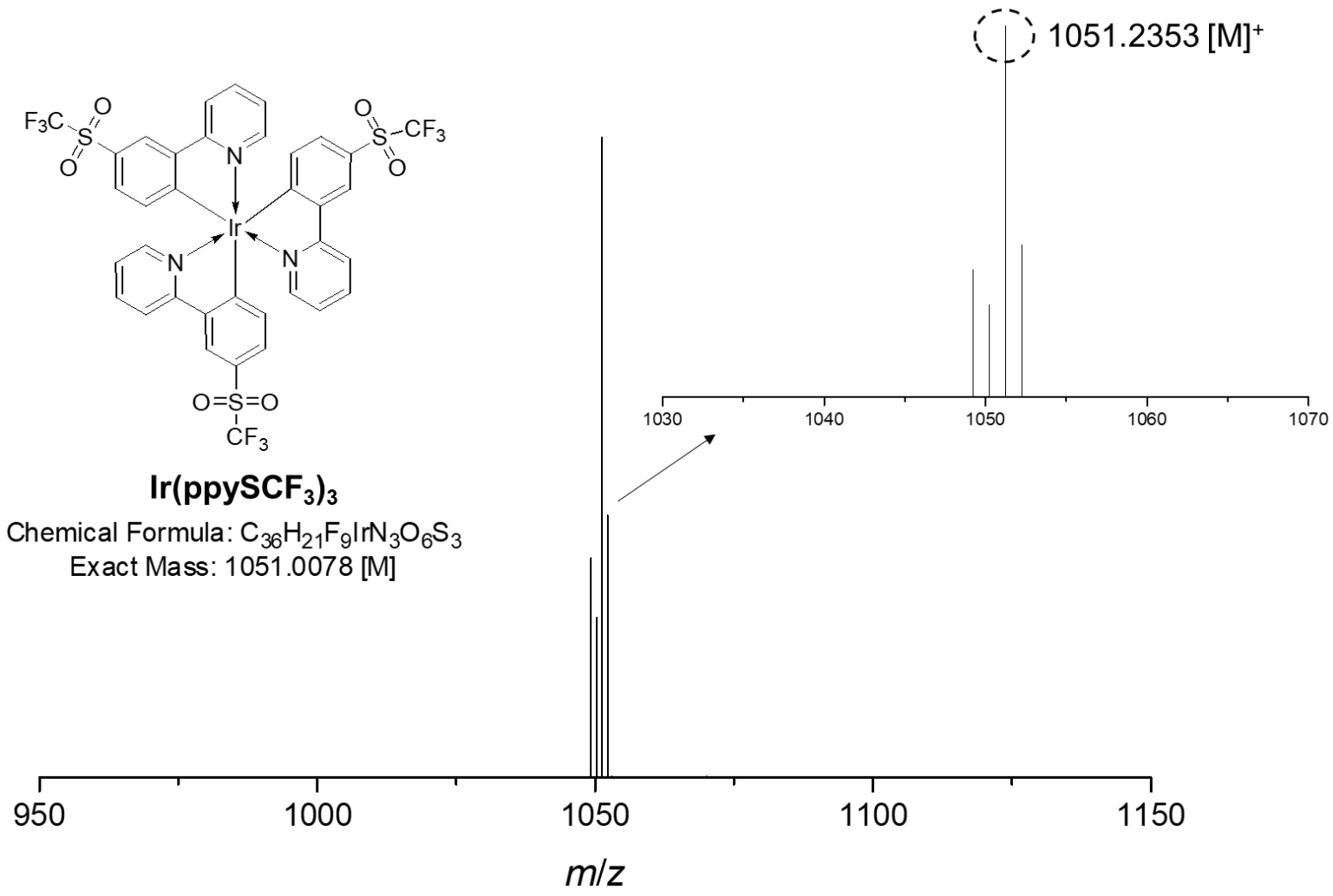

Figure S12. ESI-Mass spectra of $\operatorname{Ir}\left(\text { ppySCF }_{3}\right)_{3}$. 


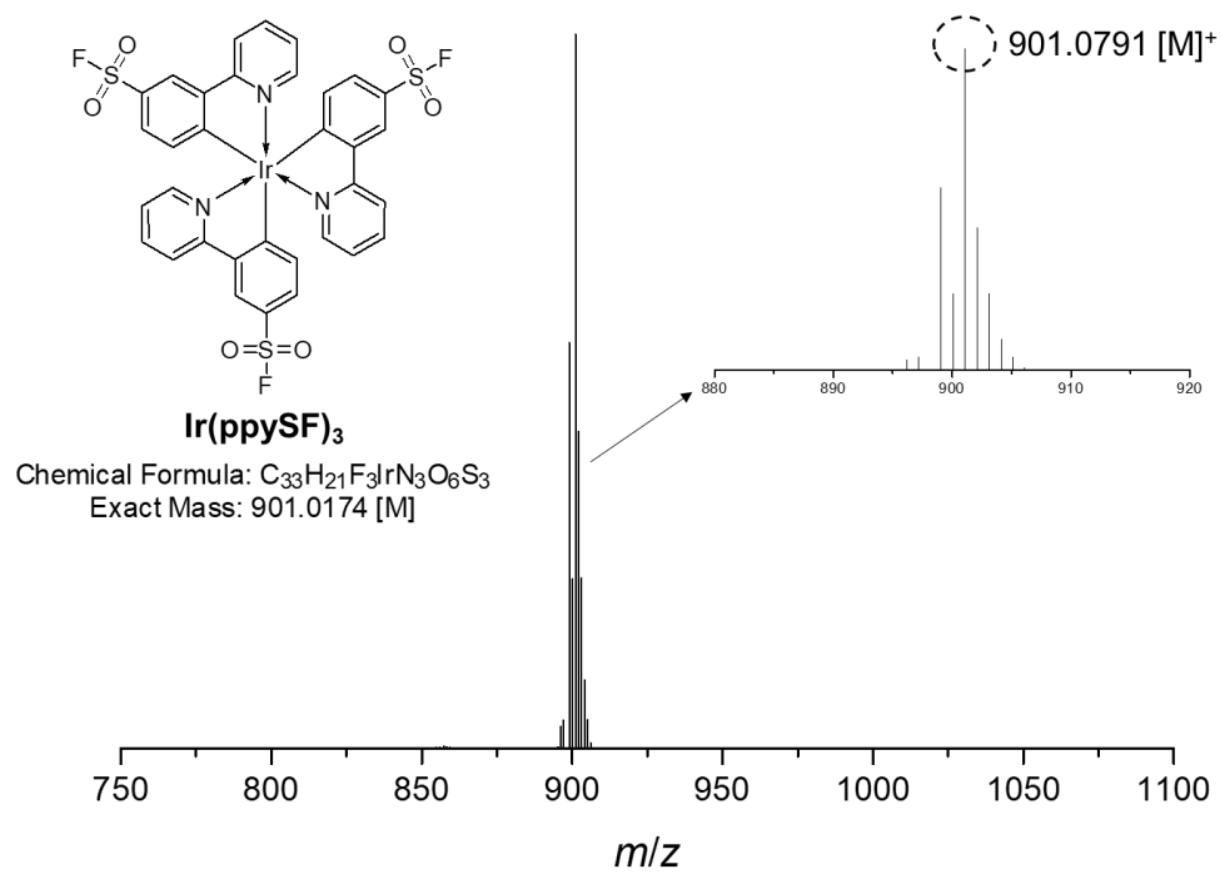

Figure S13. ESI-Mass spectra of $\operatorname{Ir}($ ppySF)3. 


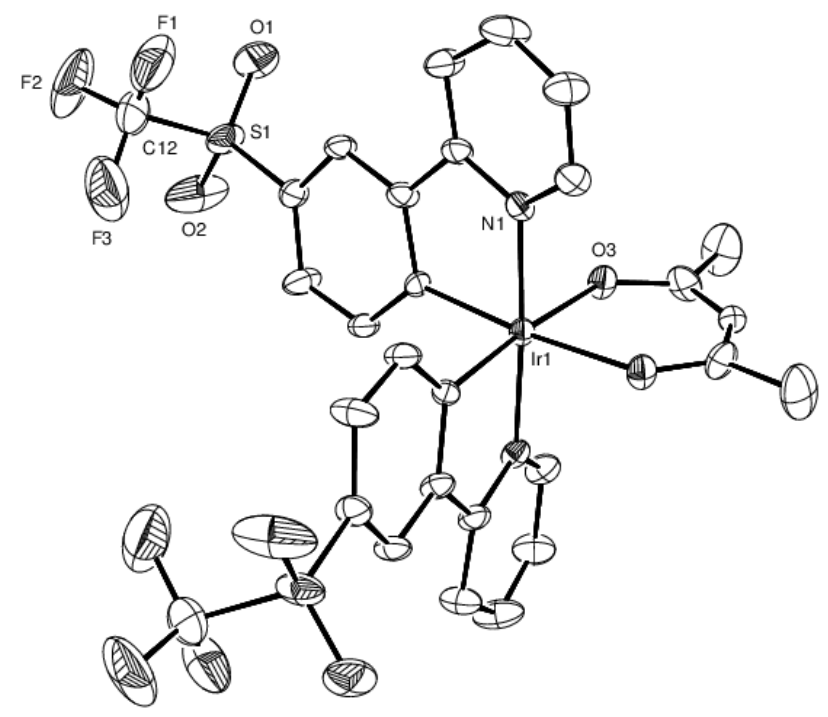

Figure S14. ORTEP drawing of $\operatorname{Ir}\left(\mathbf{p p y S C F}_{3}\right)_{2}$ (acac) with $30 \%$ probability for the thermal ellipsoids. Hydrogens were omitted for clarity. ${ }^{\mathrm{S} 9}$

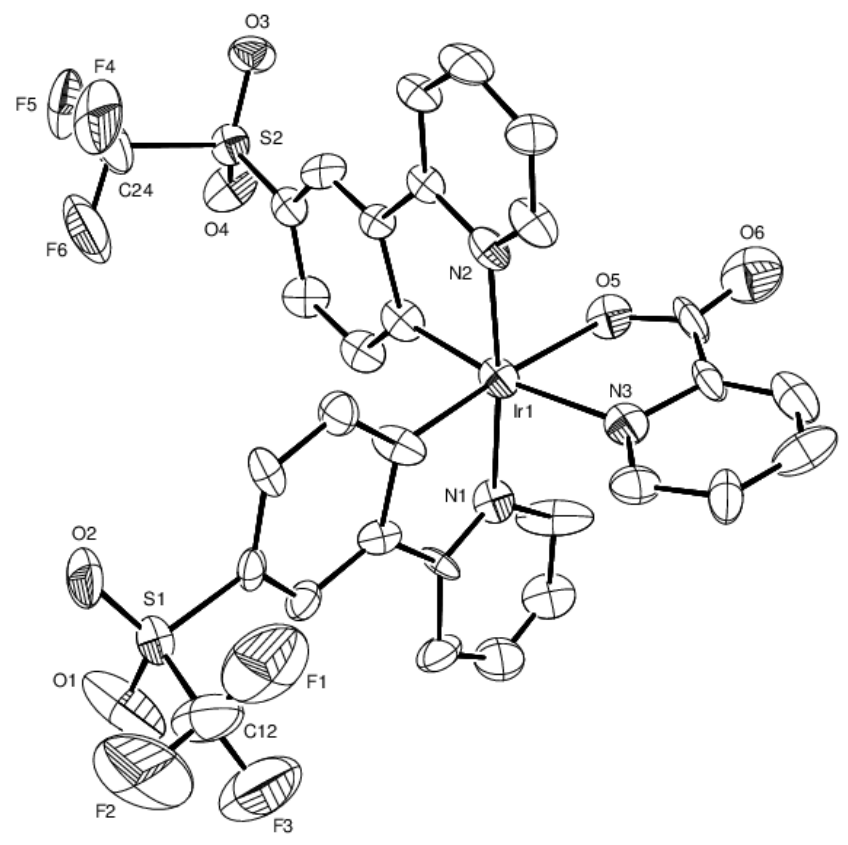

Figure S15. ORTEP drawing of $\operatorname{Ir}\left(\mathbf{p p y S C F}_{3}\right)_{2}(\mathbf{p i c})$ with $30 \%$ probability for the thermal ellipsoids. Hydrogens were omitted for clarity. ${ }^{\text {S9 }}$ 


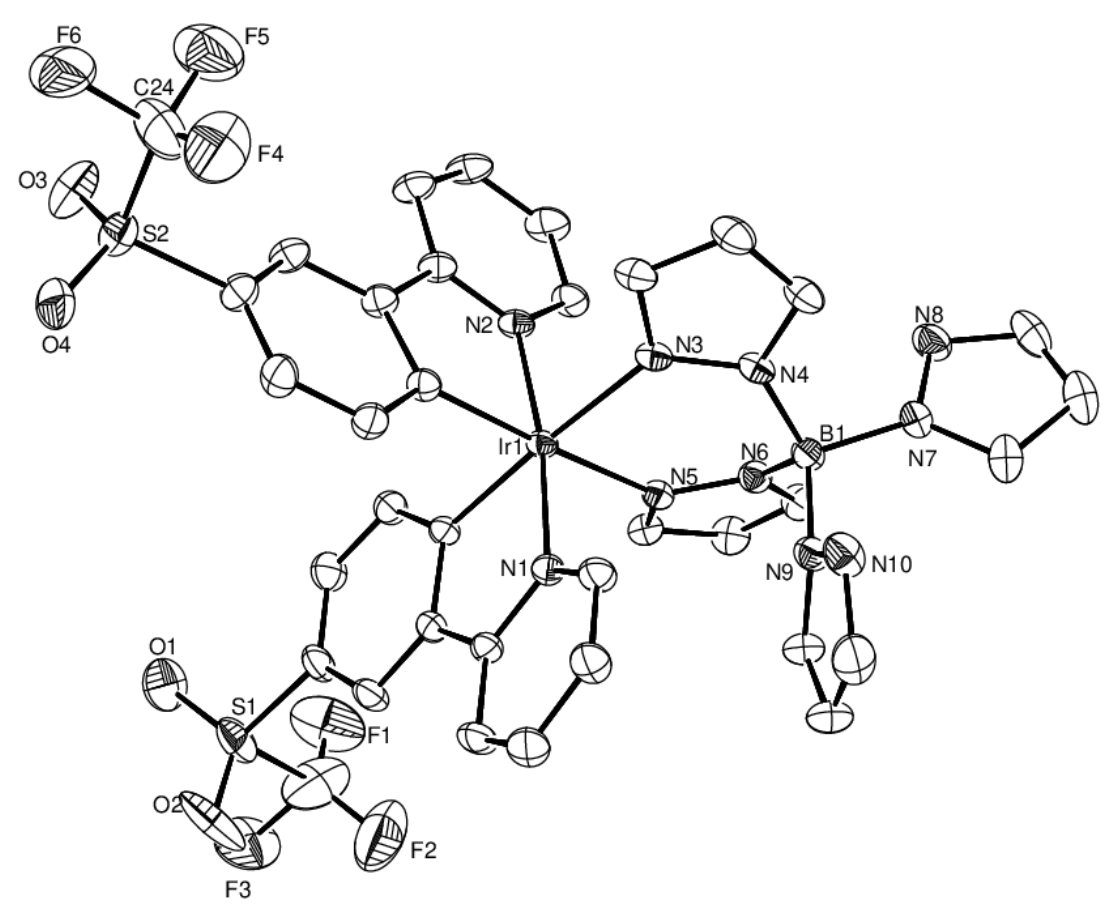

Figure S16. ORTEP drawing of $\operatorname{Ir}\left(\mathbf{p p y S C F}_{3}\right)_{2}$ (bor) with $30 \%$ probability for the thermal ellipsoids. Hydrogens were omitted for clarity. ${ }^{\text {s9 }}$

Table S1. The frontier molecular orbital contribution on main ligand, ancillary ligand, and iridium metal (Ir) in $\operatorname{Ir}(\mathrm{III})$ complexes.

\begin{tabular}{|c|c|c|c|c|c|c|c|c|c|}
\hline \multirow{2}{*}{\multicolumn{2}{|c|}{ Orbital }} & \multicolumn{4}{|c|}{$\operatorname{Ir}\left(\text { ppySCF }_{3}\right)_{3}$} & \multicolumn{4}{|c|}{$\operatorname{Ir}(\text { ppySF })_{3}$} \\
\hline & & \multicolumn{2}{|l|}{ Ligand (\%) } & \multicolumn{2}{|c|}{ Iridium $(\%)$} & \multicolumn{2}{|c|}{ Ligand (\%) } & \multicolumn{2}{|c|}{ Iridium $(\%)$} \\
\hline \multicolumn{2}{|c|}{ LUMO } & \multicolumn{2}{|l|}{98} & \multicolumn{2}{|l|}{2} & \multicolumn{2}{|c|}{98} & \multicolumn{2}{|c|}{2} \\
\hline \multicolumn{2}{|c|}{ HOMO } & \multicolumn{2}{|l|}{42} & \multicolumn{2}{|l|}{58} & \multicolumn{2}{|c|}{43} & \multicolumn{2}{|c|}{57} \\
\hline \multirow[b]{2}{*}{ Orbital } & \multicolumn{3}{|c|}{$\operatorname{Ir}\left(\text { ppySCF }_{3}\right)_{2}($ acac $)$} & \multicolumn{3}{|c|}{$\operatorname{Ir}\left(\text { ppySCF }_{3}\right)_{2}($ pic $)$} & \multicolumn{3}{|c|}{$\operatorname{Ir}\left(\text { ppySCF}_{3}\right)_{2}$ (bor) } \\
\hline & $\begin{array}{c}\text { Main } \\
\text { Ligand } \\
(\%)\end{array}$ & $\begin{array}{l}\text { Ancillary } \\
\text { Ligand }\end{array}$ & $\begin{array}{l}\text { Iridium } \\
(\%)\end{array}$ & $\begin{array}{c}\text { Main } \\
\text { Ligand } \\
(\%)\end{array}$ & $\begin{array}{l}\text { Ancillary } \\
\text { Ligand }\end{array}$ & $\begin{array}{l}\text { Iridium } \\
(\%)\end{array}$ & $\begin{array}{c}\text { Main } \\
\text { Ligand } \\
(\%)\end{array}$ & $\begin{array}{c}\text { Ancillary } \\
\text { Ligand }\end{array}$ & $\begin{array}{c}\text { Iridium } \\
(\%)\end{array}$ \\
\hline LUMO & 94 & 1 & 5 & 91 & 5 & 4 & 93 & 3 & 4 \\
\hline HOMO & 42 & 5 & 53 & 42 & 9 & 49 & 38 & 9 & 53 \\
\hline
\end{tabular}




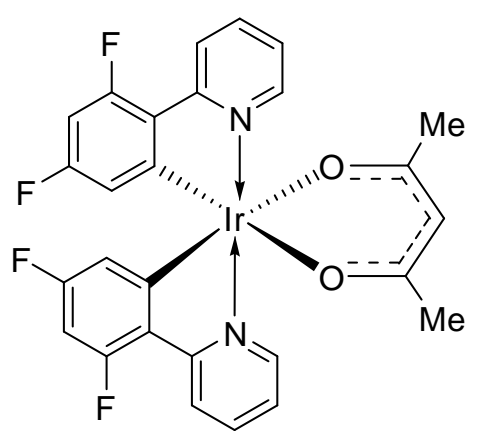

$\operatorname{FIr}(\mathrm{acac})$

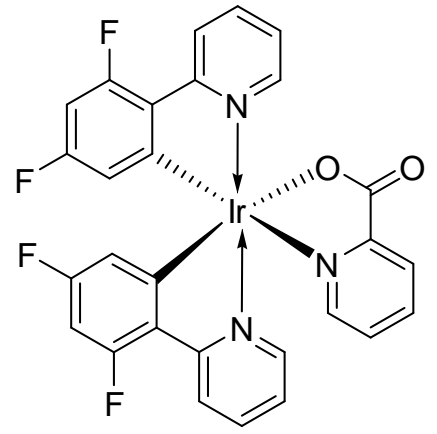

FIr(pic)

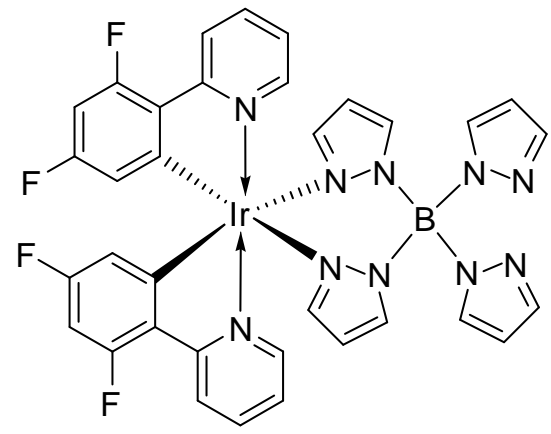

FIr(bor) (Flr6)

Figure S17. Chemical structures of FIr(acac), FIr(pic), and FIr(bor) (FIr6). 

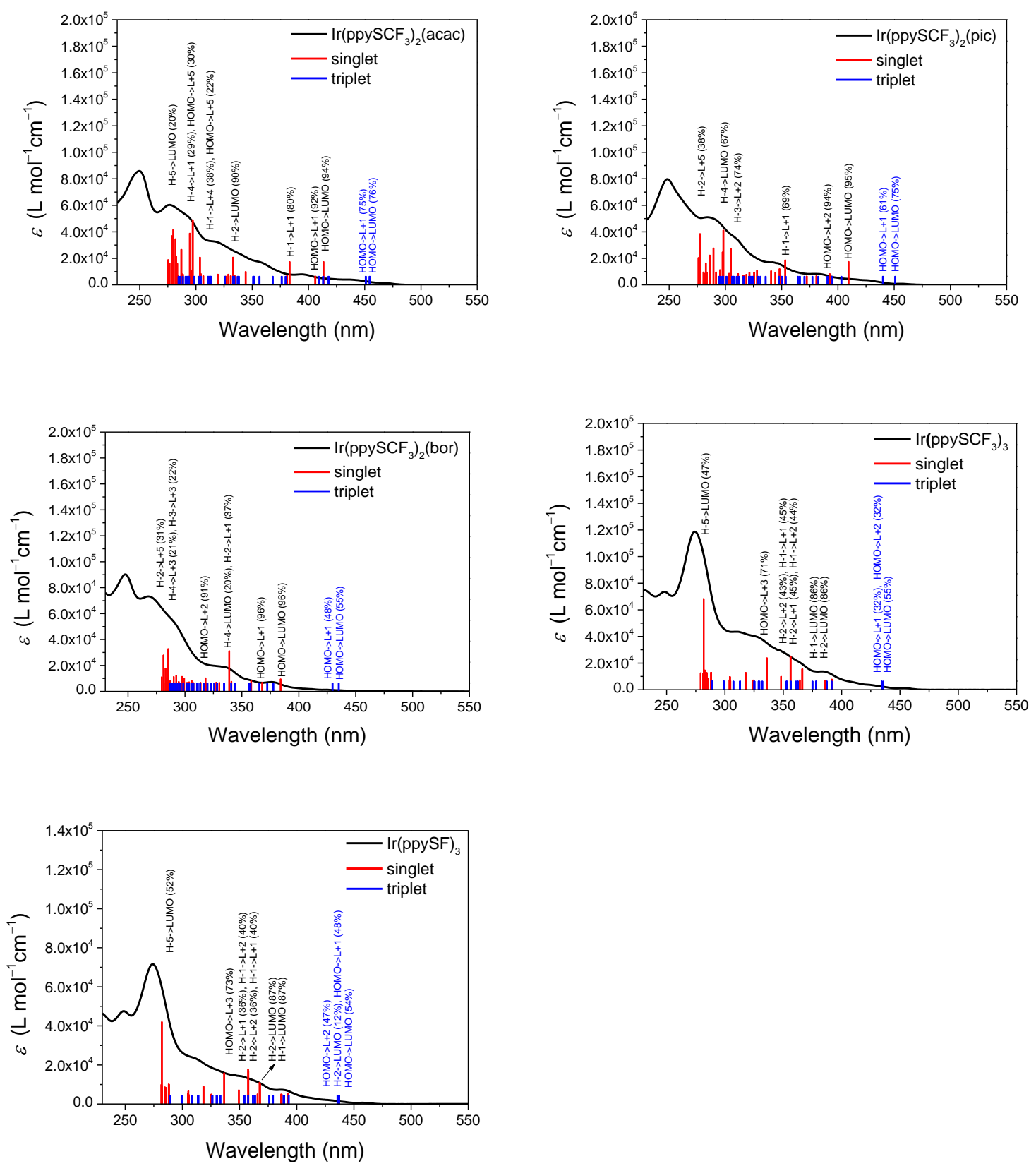

Figure S18. Experimental UV-Vis absorption spectra for Ir complexes (black lines) and theoretically calculated oscillator strengths obtained by the TD-DFT calculation (red and blue bars are for singlet and triplet states, respectively). 


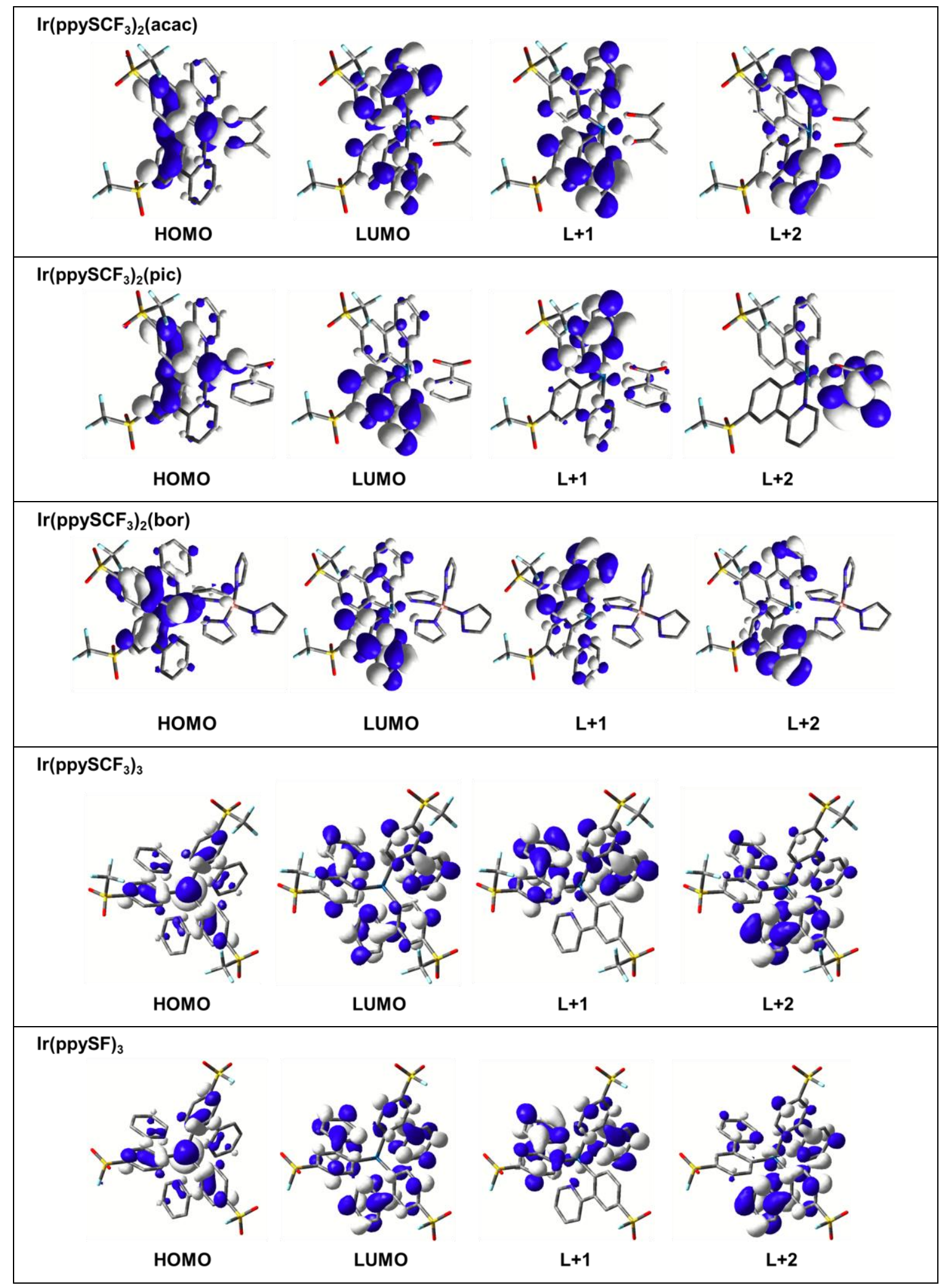

Figure S19. Spatial distributions for the frontier orbitals of $\operatorname{Ir}\left(\operatorname{ppySCF}_{3}\right)_{2}$ (acac), $\operatorname{Ir}\left(p_{p y S C F}\right)_{2}\left(\right.$ pic $_{1}, \operatorname{Ir}\left(\text { ppySCF}_{3}\right)_{2}($ bor $), \operatorname{Ir}\left(\operatorname{ppySCF}_{3}\right)_{3}$, and $\operatorname{Ir}(\text { ppySF })_{3}$. 
Table S2. Energy levels and contributions of the lower lying transitions of $\operatorname{Ir}\left(\mathbf{p p y S C F} \mathbf{S}_{\mathbf{2}} \mathbf{2}(\mathbf{a c a c})\right.$ calculated from the TD-DFT calculation.

\begin{tabular}{|c|c|c|c|c|}
\hline No. & Wavelength (nm) & Osc. Strength & Symmetry & Major contributions \\
\hline 2 & 450.9171989 & 0 & Triplet-A & $\mathrm{H}-3 \rightarrow \mathrm{LUMO}(10 \%), \mathrm{HOMO} \rightarrow \mathrm{L}+1(75 \%)$ \\
\hline 4 & 413.3081973 & 0.0345 & Singlet-A & HOMO $\rightarrow$ LUMO $(94 \%)$ \\
\hline 5 & 413.0328237 & 0 & Triplet-A & $\mathrm{H}-3 \rightarrow \mathrm{LUMO}(10 \%), \mathrm{H}-1 \rightarrow \mathrm{LUMO}(55 \%), \mathrm{HOMO} \rightarrow \mathrm{L}+1(10 \%)$ \\
\hline 6 & 409.2833097 & 0 & Triplet-A & $\mathrm{H}-1 \rightarrow \mathrm{L}+1(50 \%)$ \\
\hline 9 & 381.0208759 & 0.0024 & Singlet-A & $\mathrm{H}-1 \rightarrow \mathrm{LUMO}(78 \%), \mathrm{H}-1 \rightarrow \mathrm{L}+1(14 \%)$ \\
\hline 10 & 379.7488224 & 0 & Triplet-A & $\mathrm{H}-3 \rightarrow$ LUMO $(14 \%), \mathrm{H}-1 \rightarrow$ LUMO $(32 \%)$ \\
\hline 11 & 376.2112908 & 0 & Triplet-A & $\mathrm{H}-5 \rightarrow \mathrm{LUMO}(13 \%), \mathrm{H}-3 \rightarrow \mathrm{L}+1(12 \%), \mathrm{H}-2 \rightarrow \mathrm{LUMO}(10 \%), \mathrm{H}-1 \rightarrow \mathrm{L}+1(31 \%)$ \\
\hline 12 & 368.1896805 & 0 & Triplet-A & $\mathrm{HOMO} \rightarrow \mathrm{L}+2(74 \%)$ \\
\hline 13 & 356.5120425 & 0 & Triplet-A & $\mathrm{HOMO} \rightarrow \mathrm{L}+3(40 \%), \mathrm{HOMO} \rightarrow \mathrm{L}+6(10 \%)$ \\
\hline 14 & 351.5586611 & 0 & Triplet-A & $\mathrm{H}-2 \rightarrow \mathrm{LUMO}(38 \%), \mathrm{H}-2 \rightarrow \mathrm{L}+1(15 \%), \mathrm{HOMO} \rightarrow \mathrm{L}+3(15 \%)$ \\
\hline 20 & 334.0001428 & 0 & Triplet-A & $\mathrm{HOMO} \rightarrow \mathrm{L}+4(57 \%), \mathrm{HOMO} \rightarrow \mathrm{L}+6(28 \%)$ \\
\hline 21 & 333.0312203 & 0 & Triplet-A & $\mathrm{H}-2 \rightarrow \mathrm{L}+1(11 \%), \mathrm{H}-1 \rightarrow \mathrm{L}+2(71 \%)$ \\
\hline 22 & 332.9954422 & 0.0446 & Singlet-A & $\mathrm{H}-2 \rightarrow \operatorname{LUMO}(90 \%)$ \\
\hline 23 & 330.9598874 & 0.0011 & Singlet-A & $\mathrm{H}-2 \rightarrow \mathrm{L}+1(50 \%), \mathrm{H}-1 \rightarrow \mathrm{L}+2(12 \%), \mathrm{HOMO} \rightarrow \mathrm{L}+4(26 \%)$ \\
\hline 24 & 328.8007664 & 0.005 & Singlet-A & $\mathrm{H}-2 \rightarrow \mathrm{L}+1(11 \%), \mathrm{H}-1 \rightarrow \mathrm{L}+2(13 \%), \mathrm{HOMO} \rightarrow \mathrm{L}+4(70 \%)$ \\
\hline 25 & 326.4116286 & 0.0002 & Singlet-A & $\mathrm{H}-2 \rightarrow \mathrm{L}+1(19 \%), \mathrm{H}-1 \rightarrow \mathrm{L}+2(70 \%)$ \\
\hline 26 & 325.7170446 & 0 & Triplet-A & $\mathrm{H}-1 \rightarrow \mathrm{L}+3(71 \%)$ \\
\hline 27 & 319.464553 & 0.005 & Singlet-A & $\mathrm{H}-1 \rightarrow \mathrm{L}+3(95 \%)$ \\
\hline 28 & 313.4158927 & 0 & Triplet-A & $\mathrm{H}-5 \rightarrow \mathrm{L}+3(12 \%), \mathrm{H}-4 \rightarrow \mathrm{LUMO}(33 \%), \mathrm{H}-3 \rightarrow \mathrm{L}+2(13 \%)$ \\
\hline 29 & 312.8622802 & 0 & Triplet-A & $\mathrm{H}-5 \rightarrow \mathrm{L}+2(17 \%), \mathrm{H}-4 \rightarrow \mathrm{L}+1(24 \%), \mathrm{H}-3 \rightarrow \mathrm{L}+3(11 \%)$ \\
\hline 30 & 311.7765811 & 0 & Triplet-A & $\mathrm{H}-4 \rightarrow \mathrm{L}+1(20 \%), \mathrm{H}-3 \rightarrow \mathrm{L}+1(20 \%)$ \\
\hline 31 & 310.7139639 & 0 & Triplet-A & $\mathrm{H}-4 \rightarrow \mathrm{LUMO}(15 \%), \mathrm{H}-3 \rightarrow \mathrm{LUMO}(23 \%)$ \\
\hline 40 & 294.5692398 & 0.1007 & Singlet-A & $\mathrm{H}-4 \rightarrow \mathrm{L}+1(27 \%), \mathrm{H}-3 \rightarrow \mathrm{L}+1(33 \%), \mathrm{H}-2 \rightarrow \mathrm{L}+2(13 \%), \mathrm{HOMO} \rightarrow \mathrm{L}+5(14 \%)$ \\
\hline 41 & 293.8083675 & 0 & Triplet-A & $\mathrm{H}-2 \rightarrow \mathrm{L}+2(62 \%)$ \\
\hline 42 & 293.1900137 & 0 & Triplet-A & $\mathrm{H}-1 \rightarrow \mathrm{L}+5(54 \%)$ \\
\hline 43 & 292.1261793 & 0 & Triplet-A & $\mathrm{H}-1 \rightarrow \mathrm{L}+5(10 \%), \mathrm{H}-1 \rightarrow \mathrm{L}+6(36 \%), \mathrm{H}-1 \rightarrow \mathrm{L}+9(10 \%)$ \\
\hline 44 & 290.8924804 & 0 & Triplet-A & $\mathrm{H}-2 \rightarrow \mathrm{L}+3(17 \%), \mathrm{H}-2 \rightarrow \mathrm{L}+4(69 \%)$ \\
\hline 45 & 288.4158207 & 0.0048 & Singlet-A & $\mathrm{H}-5 \rightarrow \mathrm{L}+1(15 \%), \mathrm{H}-1 \rightarrow \mathrm{L}+5(60 \%)$ \\
\hline 46 & 288.3353326 & 0 & Triplet-A & $\mathrm{H}-2 \rightarrow \mathrm{L}+3(45 \%), \mathrm{H}-2 \rightarrow \mathrm{L}+4(16 \%), \mathrm{H}-1 \rightarrow \mathrm{L}+5(12 \%)$ \\
\hline 47 & 287.6663411 & 0.0056 & Singlet-A & $\mathrm{H}-5 \rightarrow \mathrm{L}+1(24 \%), \mathrm{H}-2 \rightarrow \mathrm{L}+3(15 \%), \mathrm{H}-1 \rightarrow \mathrm{L}+5(25 \%), \mathrm{HOMO} \rightarrow \mathrm{L}+6(14 \%)$ \\
\hline 48 & 287.0403135 & 0.0629 & Singlet-A & $\mathrm{H}-5 \rightarrow \mathrm{LUMO}(11 \%), \mathrm{H}-2 \rightarrow \mathrm{L}+2(57 \%)$ \\
\hline 49 & 286.0667567 & 0 & Triplet-A & $\mathrm{H}-7 \rightarrow \mathrm{LUMO}(10 \%), \mathrm{H}-6 \rightarrow \mathrm{LUMO}(22 \%), \mathrm{HOMO} \rightarrow \mathrm{L}+9(42 \%)$ \\
\hline 50 & 284.6089411 & 0 & Triplet-A & $\mathrm{H}-6 \rightarrow \mathrm{LUMO}(43 \%), \mathrm{HOMO} \rightarrow \mathrm{L}+9(17 \%)$ \\
\hline 51 & 284.1197878 & 0.0026 & Singlet-A & $\mathrm{H}-1 \rightarrow \mathrm{L}+6(78 \%)$ \\
\hline 52 & 283.3794867 & 0.0299 & Singlet-A & $\mathrm{H}-6 \rightarrow \mathrm{LUMO}(62 \%), \mathrm{H}-2 \rightarrow \mathrm{L}+3(16 \%)$ \\
\hline 53 & 282.385535 & 0.0476 & Singlet-A & $\mathrm{H}-6 \rightarrow \mathrm{L}+1(51 \%), \mathrm{H}-5 \rightarrow \mathrm{LUMO}(32 \%)$ \\
\hline 54 & 281.7694491 & 0.0876 & Singlet-A & $\mathrm{H}-5 \rightarrow \mathrm{L}+1(14 \%), \mathrm{H}-2 \rightarrow \mathrm{L}+3(12 \%), \mathrm{H}-2 \rightarrow \mathrm{L}+4(30 \%), \mathrm{HOMO} \rightarrow \mathrm{L}+6(16 \%)$ \\
\hline 55 & 279.8677073 & 0.1091 & Singlet-A & $\mathrm{H}-6 \rightarrow \mathrm{L}+1(13 \%), \mathrm{H}-5 \rightarrow \mathrm{LUMO}(20 \%), \mathrm{H}-2 \rightarrow \mathrm{L}+2(10 \%), \mathrm{H}-2 \rightarrow \mathrm{L}+3(10 \%)$ \\
\hline 56 & 278.5347944 & 0.0949 & Singlet-A & $\mathrm{H}-5 \rightarrow \mathrm{L}+1(22 \%), \mathrm{H}-2 \rightarrow \mathrm{L}+3(23 \%)$ \\
\hline 57 & 276.5652309 & 0.0304 & Singlet-A & $\mathrm{H}-6 \rightarrow \mathrm{L}+4(38 \%), \mathrm{H}-4 \rightarrow \mathrm{L}+4(36 \%)$ \\
\hline 58 & 275.3491006 & 0.0389 & Singlet-A & $\mathrm{H}-7 \rightarrow \mathrm{LUMO}(40 \%), \mathrm{H}-3 \rightarrow \mathrm{L}+2(25 \%), \mathrm{H}-2 \rightarrow \mathrm{L}+4(20 \%)$ \\
\hline 59 & 274.9643898 & 0.0191 & Singlet-A & $\mathrm{H}-7 \rightarrow \mathrm{L}+1(74 \%)$ \\
\hline 60 & 274.6598281 & 0.0035 & Singlet-A & $\mathrm{H}-4 \rightarrow \mathrm{L}+2(22 \%), \mathrm{H}-2 \rightarrow \mathrm{L}+4(32 \%), \mathrm{HOMO} \rightarrow \mathrm{L}+6(13 \%)$ \\
\hline
\end{tabular}


Table S3. Energy levels and contributions of the lower lying transitions of $\operatorname{Ir}\left(\mathbf{p p y S C F} \mathbf{S F}_{\mathbf{3}} \mathbf{2}_{\mathbf{2}}\right.$ (pic) calculated from the TD-DFT calculation.

\begin{tabular}{|c|c|c|c|c|}
\hline No. & Wavelength $(\mathrm{nm})$ & Osc. Strength & Symmetry & Major contributions \\
\hline 1 & 450.95 & $\mathbf{0}$ & Triplet-A & HOMO $\rightarrow$ LUMO $(75 \%)$ \\
\hline 2 & 440.01914 & 0 & Triplet-A & $\mathrm{H}-4 \rightarrow \mathrm{L}+1(10 \%), \mathrm{HOMO} \rightarrow \mathrm{L}+1(61 \%)$ \\
\hline 3 & 409.391425 & 0.0347 & Singlet-A & HOMO $\rightarrow$ LUMO $(95 \%)$ \\
\hline 4 & 403.043343 & 0 & Triplet-A & $\mathrm{H}-1 \rightarrow \mathrm{L}+1(37 \%), \mathrm{HOMO} \rightarrow \mathrm{L}+1(24 \%)$ \\
\hline 5 & 395.068008 & 0 & Triplet-A & $\mathrm{H}-5 \rightarrow \mathrm{LUMO}(10 \%), \mathrm{H}-3 \rightarrow$ LUMO $(30 \%), \mathrm{HOMO} \rightarrow$ LUMO $(12 \%)$ \\
\hline 6 & 392.740324 & 0.006 & Singlet-A & HOMO $\rightarrow$ L+1 $(94 \%)$ \\
\hline 7 & 390.772167 & 0 & Triplet-A & $\mathrm{H}-2 \rightarrow \mathrm{L}+2(17 \%), \mathrm{HOMO} \rightarrow \mathrm{L}+2(56 \%)$ \\
\hline 8 & 382.596411 & 0 & Triplet-A & $\mathrm{H}-1 \rightarrow \mathrm{L}+2(68 \%)$ \\
\hline 9 & 380.927224 & 0.0013 & Singlet-A & $\mathrm{HOMO} \rightarrow \mathrm{L}+2(94 \%)$ \\
\hline 10 & 377.321869 & 0 & Triplet-A & $\mathrm{H}-1 \rightarrow \mathrm{LUMO}(77 \%)$ \\
\hline 11 & 372.313723 & 0.0006 & Singlet-A & $\mathrm{H}-1 \rightarrow \mathrm{LUMO}(91 \%)$ \\
\hline 12 & 370.26786 & 0 & Triplet-A & $\mathrm{H}-2 \rightarrow \mathrm{L}+2(54 \%), \mathrm{HOMO} \rightarrow \mathrm{L}+2(20 \%)$ \\
\hline 13 & 366.156324 & 0 & Triplet-A & $\mathrm{HOMO} \rightarrow \mathrm{L}+3(65 \%)$ \\
\hline 14 & 364.337917 & 0 & Triplet-A & $\mathrm{H}-4 \rightarrow \mathrm{L}+1(23 \%), \mathrm{H}-1 \rightarrow \mathrm{L}+1(26 \%)$ \\
\hline 15 & 353.56373 & 0 & Triplet-A & $\mathrm{H}-5 \rightarrow \mathrm{LUMO}(16 \%), \mathrm{H}-3 \rightarrow$ LUMO $(18 \%)$ \\
\hline 16 & 353.181008 & 0.0383 & Singlet-A & $\mathrm{H}-1 \rightarrow \mathrm{L}+1(69 \%), \mathrm{H}-1 \rightarrow \mathrm{L}+2(16 \%)$ \\
\hline 17 & 350.069721 & 0 & Triplet-A & $\mathrm{HOMO} \rightarrow \mathrm{L}+4(56 \%)$ \\
\hline 18 & 348.11375 & 0.0176 & Singlet-A & $\mathrm{H}-2 \rightarrow \mathrm{L}+2(30 \%), \mathrm{H}-1 \rightarrow \mathrm{L}+1(16 \%), \mathrm{H}-1 \rightarrow \mathrm{L}+2(35 \%)$ \\
\hline 19 & 347.547774 & 0 & Triplet-A & $\mathrm{H}-3 \rightarrow \mathrm{LUMO}(10 \%), \mathrm{H}-3 \rightarrow \mathrm{L}+2(35 \%)$ \\
\hline 20 & 344.371839 & 0.0097 & Singlet-A & $\mathrm{HOMO} \rightarrow \mathrm{L}+3(94 \%)$ \\
\hline 21 & 340.625273 & 0.0128 & Singlet-A & $\mathrm{H}-2 \rightarrow \mathrm{L}+2(39 \%), \mathrm{H}-1 \rightarrow \mathrm{L}+2(44 \%)$ \\
\hline 22 & 335.800317 & 0 & Triplet-A & $\mathrm{H}-5 \rightarrow \mathrm{LUMO}(14 \%), \mathrm{HOMO} \rightarrow \mathrm{L}+6(53 \%)$ \\
\hline 23 & 331.154362 & 0 & Triplet-A & $\mathrm{HOMO} \rightarrow \mathrm{L}+7(58 \%)$ \\
\hline 24 & 328.975252 & 0 & Triplet-A & $\mathrm{H}-3 \rightarrow \mathrm{L}+1(77 \%)$ \\
\hline 25 & 328.095988 & 0.0145 & Singlet-A & $\mathrm{H}-3 \rightarrow \mathrm{LUMO}(26 \%), \mathrm{HOMO} \rightarrow \mathrm{L}+4(67 \%)$ \\
\hline 26 & 325.469084 & 0.0093 & Singlet-A & $\mathrm{H}-3 \rightarrow \mathrm{LUMO}(44 \%), \mathrm{H}-3 \rightarrow \mathrm{L}+1(23 \%), \mathrm{HOMO} \rightarrow \mathrm{L}+4(28 \%)$ \\
\hline 27 & 324.328223 & 0 & Triplet-A & $\mathrm{H}-1 \rightarrow \mathrm{L}+3(53 \%), \mathrm{HOMO} \rightarrow \mathrm{L}+5(12 \%)$ \\
\hline 28 & 322.57309 & 0 & Triplet-A & $\mathrm{H}-2 \rightarrow \mathrm{LUMO}(48 \%), \mathrm{HOMO} \rightarrow \mathrm{L}+5(21 \%)$ \\
\hline 29 & 321.394077 & 0.0088 & Singlet-A & $\mathrm{H}-3 \rightarrow \mathrm{LUMO}(18 \%), \mathrm{H}-3 \rightarrow \mathrm{L}+1(62 \%)$ \\
\hline 30 & 321.086117 & 0 & Triplet-A & $\mathrm{H}-2 \rightarrow \mathrm{LUMO}(24 \%), \mathrm{H}-1 \rightarrow \mathrm{L}+3(22 \%), \mathrm{HOMO} \rightarrow \mathrm{L}+5(30 \%)$ \\
\hline 31 & 318.725432 & 0.0047 & Singlet-A & $\mathrm{H}-2 \rightarrow \mathrm{LUMO}(78 \%), \mathrm{H}-1 \rightarrow \mathrm{L}+3(10 \%)$ \\
\hline 32 & 316.795342 & 0.0021 & Singlet-A & $\mathrm{H}-1 \rightarrow \mathrm{L}+3(71 \%), \mathrm{HOMO} \rightarrow \mathrm{L}+5(12 \%)$ \\
\hline 33 & 316.108799 & 0 & Triplet-A & $\mathrm{H}-2 \rightarrow \mathrm{L}+1(59 \%)$ \\
\hline 34 & 315.794791 & 0.0024 & Singlet-A & $\mathrm{H}-1 \rightarrow \mathrm{L}+3(11 \%), \mathrm{HOMO} \rightarrow \mathrm{L}+5(84 \%)$ \\
\hline 35 & 311.815787 & 0 & Triplet-A & $\mathrm{H}-1 \rightarrow \mathrm{L}+4(34 \%), \mathrm{H}-1 \rightarrow \mathrm{L}+5(19 \%)$ \\
\hline 36 & 311.299069 & 0.0065 & Singlet-A & $\mathrm{H}-2 \rightarrow \mathrm{L}+1(82 \%)$ \\
\hline 37 & 310.092272 & 0 & Triplet-A & $\mathrm{H}-5 \rightarrow \mathrm{L}+3(13 \%)$ \\
\hline 38 & 307.370883 & 0 & Triplet-A & $\mathrm{H}-1 \rightarrow \mathrm{L}+4(22 \%), \mathrm{H}-1 \rightarrow \mathrm{L}+5(20 \%)$ \\
\hline 39 & 306.800438 & 0 & Triplet-A & $\mathrm{H}-6 \rightarrow$ LUMO $(10 \%), \mathrm{H}-4 \rightarrow$ LUMO $(18 \%), \mathrm{H}-3 \rightarrow \mathrm{L}+3(16 \%)$ \\
\hline 40 & 305.861933 & 0 & Triplet-A & $\mathrm{H}-4 \rightarrow \mathrm{L}+1(13 \%), \mathrm{H}-2 \rightarrow \mathrm{L}+1(18 \%), \mathrm{H}-1 \rightarrow \mathrm{L}+4(10 \%)$ \\
\hline 41 & 304.951652 & 0.0642 & Singlet-A & $\mathrm{H}-3 \rightarrow \mathrm{L}+2(74 \%), \mathrm{HOMO} \rightarrow \mathrm{L}+6(11 \%)$ \\
\hline 42 & 303.310402 & 0.0069 & Singlet-A & $\mathrm{H}-4 \rightarrow \mathrm{L}+1(10 \%), \mathrm{H}-1 \rightarrow \mathrm{L}+4(76 \%)$ \\
\hline 43 & 301.027492 & 0 & Triplet-A & $\mathrm{H}-10 \rightarrow \mathrm{L}+5(18 \%), \mathrm{H}-3 \rightarrow \mathrm{L}+2(26 \%), \mathrm{H}-1 \rightarrow \mathrm{L}+5(11 \%)$ \\
\hline 44 & 298.203798 & 0.1079 & Singlet-A & $\mathrm{H}-4 \rightarrow \mathrm{LUMO}(13 \%), \mathrm{HOMO} \rightarrow \mathrm{L}+6(42 \%)$ \\
\hline 45 & 297.995945 & 0 & Triplet-A & $\mathrm{H}-10 \rightarrow \mathrm{L}+2(21 \%), \mathrm{H}-3 \rightarrow \mathrm{L}+3(15 \%), \mathrm{H}-1 \rightarrow \mathrm{L}+5(13 \%)$ \\
\hline 46 & 297.509702 & 0.0569 & Singlet-A & $\mathrm{H}-4 \rightarrow \mathrm{LUMO}(67 \%), \mathrm{HOMO} \rightarrow \mathrm{L}+6(16 \%)$ \\
\hline 47 & 297.402655 & 0 & Triplet-A & $\mathrm{H}-5 \rightarrow \mathrm{L}+1(15 \%), \mathrm{H}-2 \rightarrow \mathrm{L}+3(13 \%)$ \\
\hline 48 & 295.904995 & 0 & Triplet-A & $\mathrm{H}-2 \rightarrow \mathrm{L}+3(40 \%), \mathrm{H}-2 \rightarrow \mathrm{L}+5(25 \%)$ \\
\hline 49 & 295.158294 & 0.0153 & Singlet-A & $\mathrm{H}-2 \rightarrow \mathrm{L}+3(28 \%), \mathrm{H}-1 \rightarrow \mathrm{L}+5(47 \%)$ \\
\hline 50 & 294.457305 & 0 & Triplet-A & $\mathrm{H}-5 \rightarrow$ LUMO $(14 \%), \mathrm{H}-4 \rightarrow$ LUMO $(13 \%), \mathrm{H}-3 \rightarrow \mathrm{L}+3(15 \%)$ \\
\hline 51 & 291.706922 & 0.01 & Singlet-A & $\mathrm{H}-2 \rightarrow \mathrm{L}+3(39 \%), \mathrm{H}-2 \rightarrow \mathrm{L}+5(15 \%), \mathrm{H}-1 \rightarrow \mathrm{L}+5(34 \%)$ \\
\hline 52 & 289.432484 & 0.0662 & Singlet-A & $\mathrm{H}-5 \rightarrow \mathrm{LUMO}(32 \%), \mathrm{H}-3 \rightarrow \mathrm{L}+3(35 \%)$ \\
\hline 53 & 286.126172 & 0.0259 & Singlet-A & $\mathrm{H}-5 \rightarrow \mathrm{L}+1(17 \%), \mathrm{H}-4 \rightarrow \mathrm{L}+1(33 \%), \mathrm{H}-3 \rightarrow \mathrm{L}+3(14 \%)$ \\
\hline 54 & 286.099762 & 0.0495 & Singlet-A & $\mathrm{H}-6 \rightarrow \mathrm{LUMO}(18 \%), \mathrm{H}-5 \rightarrow \mathrm{LUMO}(14 \%), \mathrm{H}-4 \rightarrow \mathrm{L}+2(13 \%)$ \\
\hline 55 & 283.535019 & 0.0099 & Singlet-A & $\mathrm{H}-4 \rightarrow \mathrm{L}+2(63 \%), \mathrm{H}-1 \rightarrow \mathrm{L}+6(17 \%)$ \\
\hline 56 & 282.630147 & 0.0314 & Singlet-A & $\mathrm{H}-5 \rightarrow \mathrm{L}+1(17 \%), \mathrm{H}-4 \rightarrow \mathrm{L}+2(13 \%), \mathrm{H}-1 \rightarrow \mathrm{L}+6(36 \%)$ \\
\hline 57 & 281.334679 & 0.007 & Singlet-A & $\mathrm{H}-5 \rightarrow \mathrm{L}+1(15 \%), \mathrm{H}-3 \rightarrow \mathrm{L}+3(20 \%), \mathrm{H}-3 \rightarrow \mathrm{L}+4(16 \%), \mathrm{H}-1 \rightarrow \mathrm{L}+6(30 \%)$ \\
\hline 58 & 280.519917 & 0.01 & Singlet-A & $\mathrm{H}-7 \rightarrow \mathrm{L}+2(13 \%), \mathrm{H}-5 \rightarrow \mathrm{L}+2(65 \%)$ \\
\hline 59 & 277.468877 & 0.0996 & Singlet-A & $\mathrm{H}-6 \rightarrow \mathrm{LUMO}(14 \%), \mathrm{H}-5 \rightarrow \mathrm{L}+1(12 \%), \mathrm{H}-3 \rightarrow \mathrm{L}+4(18 \%), \mathrm{H}-2 \rightarrow \mathrm{L}+4(13 \%)$ \\
\hline 60 & 276.047987 & 0.0437 & Singlet-A & $\mathrm{H}-2 \rightarrow \mathrm{L}+3(18 \%), \mathrm{H}-2 \rightarrow \mathrm{L}+5(38 \%)$ \\
\hline
\end{tabular}


Table S4. Energy levels and contributions of the lower lying transitions of $\operatorname{Ir}\left(\mathbf{p p y S C F} 3 \mathbf{S}_{2}\right.$ (bor) calculated from the TD-DFT calculation.

\begin{tabular}{|c|c|c|c|c|}
\hline No. & Wavelength $(\mathrm{nm})$ & Osc. Strength & Symmetry & Major contributions \\
\hline 1 & 435.139132 & $\mathbf{0}$ & Triplet-A & HOMO $\rightarrow$ LUMO $(55 \%)$ \\
\hline 2 & 429.635432 & 0 & Triplet-A & $\mathrm{H}-7 \rightarrow \mathrm{LUMO}(13 \%), \mathrm{HOMO} \rightarrow \mathrm{L}+1(48 \%)$ \\
\hline 3 & 384.054125 & 0.0097 & Singlet-A & HOMO $\rightarrow$ LUMO $(96 \%)$ \\
\hline 4 & 377.597664 & 0 & Triplet-A & $\mathrm{H}-4 \rightarrow$ LUMO $(15 \%), \mathrm{H}-3 \rightarrow$ LUMO $(14 \%), \mathrm{HOMO} \rightarrow$ LUMO $(24 \%)$ \\
\hline 5 & 372.347267 & 0 & Triplet-A & $\mathrm{H}-2 \rightarrow \operatorname{LUMO}(16 \%), \mathrm{H}-2 \rightarrow \mathrm{L}+1(34 \%), \mathrm{H}-1 \rightarrow \mathrm{L}+1(12 \%)$ \\
\hline 6 & 367.85104 & 0.0017 & Singlet-A & HOMO $\rightarrow$ L+1 $(96 \%)$ \\
\hline 7 & 365.422479 & 0 & Triplet-A & $\mathrm{H}-4 \rightarrow \mathrm{LUMO}(20 \%), \mathrm{H}-2 \rightarrow$ LUMO $(20 \%), \mathrm{H}-1 \rightarrow$ LUMO $(11 \%)$ \\
\hline 8 & 358.190885 & 0.0055 & Singlet-A & H-2 $\rightarrow$ LUMO $(64 \%)$, H-1 $\rightarrow$ LUMO $(24 \%)$ \\
\hline 9 & 357.612325 & 0 & Triplet-A & $\mathrm{H}-7 \rightarrow \mathrm{L}+1(13 \%), \mathrm{HOMO} \rightarrow \mathrm{L}+1(21 \%)$ \\
\hline 10 & 356.440297 & 0 & Triplet-A & $\mathrm{H}-7 \rightarrow \mathrm{LUMO}(14 \%), \mathrm{H}-2 \rightarrow \mathrm{LUMO}(14 \%), \mathrm{HOMO} \rightarrow \mathrm{L}+1(11 \%)$ \\
\hline 11 & 343.722639 & 0 & Triplet-A & $\mathrm{HOMO} \rightarrow \mathrm{L}+2(49 \%)$ \\
\hline 12 & 340.821906 & 0.0038 & Singlet-A & $\begin{array}{l}\text { H-4 } \rightarrow \text { LUMO }(27 \%), \quad \text { H-4 } \rightarrow \text { L+1 } \quad(11 \%), \quad \text { H-3 } \rightarrow \text { LUMO } \quad(20 \%), \quad H-3 \rightarrow L+1 \\
(13 \%), \text { H-2 } \rightarrow \text { L+1 }(16 \%)\end{array}$ \\
\hline 13 & 340.662709 & 0 & Triplet-A & $\mathrm{H}-4 \rightarrow \mathrm{L}+1(35 \%), \mathrm{H}-3 \rightarrow \mathrm{L}+1(26 \%)$ \\
\hline 14 & 338.939839 & 0.0769 & Singlet-A & $\mathrm{H}-4 \rightarrow \operatorname{LUMO}(20 \%), \mathrm{H}-3 \rightarrow \mathrm{LUMO}(13 \%), \mathrm{H}-2 \rightarrow \mathrm{L}+1(37 \%), \mathrm{H}-1 \rightarrow \mathrm{L}+1(11 \%)$ \\
\hline 15 & 334.396507 & 0 & Triplet-A & $\mathrm{H}-7 \rightarrow \mathrm{L}+2(13 \%), \mathrm{HOMO} \rightarrow \mathrm{L}+3(25 \%), \mathrm{HOMO} \rightarrow \mathrm{L}+5(16 \%)$ \\
\hline 16 & 330.245833 & 0.0012 & Singlet-A & $\mathrm{H}-4 \rightarrow \mathrm{L}+1(34 \%), \mathrm{H}-3 \rightarrow \mathrm{L}+1(22 \%), \mathrm{H}-1 \rightarrow \mathrm{L}+1(15 \%)$ \\
\hline 17 & 328.269727 & 0 & Triplet-A & $\mathrm{H}-2 \rightarrow \mathrm{LUMO}(25 \%), \mathrm{H}-1 \rightarrow \mathrm{LUMO}(69 \%)$ \\
\hline 18 & 327.601842 & 0.0018 & Singlet-A & $\mathrm{H}-2 \rightarrow \mathrm{LUMO}(25 \%), \mathrm{H}-1 \rightarrow$ LUMO $(56 \%)$ \\
\hline 19 & 325.717045 & 0 & Triplet-A & $\mathrm{HOMO} \rightarrow \mathrm{L}+4(20 \%), \mathrm{HOMO} \rightarrow \mathrm{L}+5(15 \%)$ \\
\hline 20 & 322.799846 & 0 & Triplet-A & $\mathrm{HOMO} \rightarrow \mathrm{L}+4(17 \%), \mathrm{HOMO} \rightarrow \mathrm{L}+5(15 \%)$ \\
\hline 21 & 319.802401 & 0 & Triplet-A & $\mathrm{H}-3 \rightarrow \mathrm{LUMO}(21 \%), \mathrm{H}-3 \rightarrow \mathrm{L}+1(12 \%), \mathrm{H}-2 \rightarrow \mathrm{L}+1(18 \%), \mathrm{H}-1 \rightarrow \mathrm{L}+1(28 \%)$ \\
\hline 22 & 319.300008 & 0.0005 & Singlet-A & $\begin{array}{l}\mathrm{H}-4 \rightarrow \mathrm{LUMO}(12 \%), \mathrm{H}-3 \rightarrow \mathrm{LUMO}(14 \%), \mathrm{H}-3 \rightarrow \mathrm{L}+1(15 \%), \mathrm{H}-2 \rightarrow \mathrm{L}+1(19 \%), \\
\mathrm{H}-1 \rightarrow \mathrm{L}+1(32 \%)\end{array}$ \\
\hline 23 & 318.087621 & 0.0123 & Singlet-A & $\mathrm{HOMO} \rightarrow \mathrm{L}+2(91 \%)$ \\
\hline 24 & 316.520367 & 0 & Triplet-A & $\mathrm{H}-2 \rightarrow \mathrm{L}+2(11 \%), \mathrm{H}-1 \rightarrow \mathrm{L}+1(16 \%), \mathrm{HOMO} \rightarrow \mathrm{L}+2(18 \%)$ \\
\hline 25 & 313.717247 & 0.0013 & Singlet-A & $\mathrm{H}-4 \rightarrow \mathrm{LUMO}(27 \%), \mathrm{H}-3 \rightarrow \mathrm{LUMO}(37 \%), \mathrm{H}-2 \rightarrow \mathrm{L}+1(11 \%), \mathrm{H}-1 \rightarrow \mathrm{L}+1(21 \%)$ \\
\hline 26 & 313.384205 & 0 & Triplet-A & $\mathrm{H}-4 \rightarrow \mathrm{LUMO}(25 \%), \mathrm{H}-3 \rightarrow \mathrm{LUMO}(27 \%), \mathrm{H}-2 \rightarrow \mathrm{L}+1(10 \%), \mathrm{H}-1 \rightarrow \mathrm{L}+1(13 \%)$ \\
\hline 27 & 311.291253 & 0 & Triplet-A & $\mathrm{H}-7 \rightarrow \mathrm{LUMO}(10 \%), \mathrm{HOMO} \rightarrow \mathrm{L}+3(14 \%)$ \\
\hline 28 & 310.612769 & 0 & Triplet-A & $\mathrm{H}-11 \rightarrow \mathrm{LUMO}(13 \%), \mathrm{H}-7 \rightarrow \mathrm{LUMO}(18 \%), \mathrm{H}-4 \rightarrow \mathrm{L}+2(11 \%)$ \\
\hline 29 & 307.52336 & 0.0007 & Singlet-A & $\mathrm{H}-4 \rightarrow \mathrm{L}+1(32 \%), \mathrm{H}-3 \rightarrow \mathrm{L}+1(34 \%), \mathrm{H}-1 \rightarrow \mathrm{L}+1(13 \%)$ \\
\hline 30 & 307.431856 & 0 & Triplet-A & $\mathrm{H}-4 \rightarrow \mathrm{L}+1(27 \%), \mathrm{H}-3 \rightarrow \mathrm{L}+1(30 \%), \mathrm{H}-1 \rightarrow \mathrm{L}+1(12 \%)$ \\
\hline 31 & 306.005363 & 0 & Triplet-A & $\mathrm{H}-5 \rightarrow \mathrm{LUMO}(40 \%), \mathrm{H}-5 \rightarrow \mathrm{L}+1(19 \%)$ \\
\hline 32 & 305.861933 & 0.0058 & Singlet-A & $\mathrm{H}-5 \rightarrow \mathrm{LUMO}(53 \%), \mathrm{H}-5 \rightarrow \mathrm{L}+1(12 \%), \mathrm{HOMO} \rightarrow \mathrm{L}+3(11 \%)$ \\
\hline 33 & 303.644673 & 0 & Triplet-A & $\mathrm{H}-7 \rightarrow \mathrm{L}+1(21 \%), \mathrm{H}-5 \rightarrow \mathrm{LUMO}(24 \%)$ \\
\hline 34 & 303.629801 & 0.0026 & Singlet-A & $\mathrm{H}-5 \rightarrow \mathrm{LUMO}(15 \%), \mathrm{HOMO} \rightarrow \mathrm{L}+3(62 \%)$ \\
\hline 35 & 301.885057 & 0.0014 & Singlet-A & $\mathrm{H}-2 \rightarrow \mathrm{L}+2(55 \%), \mathrm{H}-1 \rightarrow \mathrm{L}+2(23 \%)$ \\
\hline 36 & 301.503315 & 0 & Triplet-A & $\mathrm{H}-2 \rightarrow \mathrm{L}+2(25 \%), \mathrm{H}-2 \rightarrow \mathrm{L}+3(12 \%), \mathrm{HOMO} \rightarrow \mathrm{L}+3(12 \%)$ \\
\hline 37 & 299.290767 & 0.0115 & Singlet-A & $\mathrm{H}-7 \rightarrow \mathrm{LUMO}(25 \%), \mathrm{H}-5 \rightarrow \mathrm{LUMO}(11 \%), \mathrm{H}-5 \rightarrow \mathrm{L}+1(43 \%)$ \\
\hline 38 & 298.584416 & 0 & Triplet-A & $\mathrm{H}-5 \rightarrow \mathrm{LUMO}(20 \%), \mathrm{H}-5 \rightarrow \mathrm{L}+1(56 \%)$ \\
\hline 39 & 297.431194 & 0.0157 & Singlet-A & $\mathrm{H}-7 \rightarrow$ LUMO $(34 \%), \mathrm{H}-5 \rightarrow$ LUMO $(11 \%), \mathrm{H}-5 \rightarrow \mathrm{L}+1(21 \%)$ \\
\hline 40 & 296.201904 & 0 & Triplet-A & $\mathrm{H}-4 \rightarrow \mathrm{L}+6(24 \%), \mathrm{H}-3 \rightarrow \mathrm{L}+6(14 \%)$ \\
\hline 41 & 295.242637 & 0 & Triplet-A & $\mathrm{H}-6 \rightarrow \mathrm{LUMO}(66 \%)$ \\
\hline 42 & 294.527254 & 0.0034 & Singlet-A & $\mathrm{H}-6 \rightarrow \mathrm{LUMO}(78 \%)$ \\
\hline 43 & 293.391214 & 0 & Triplet-A & \\
\hline 44 & 292.374176 & 0.0188 & Singlet-A & $\mathrm{H}-7 \rightarrow \mathrm{LUMO}(10 \%), \mathrm{H}-7 \rightarrow \mathrm{L}+1(12 \%), \mathrm{H}-4 \rightarrow \mathrm{L}+2(26 \%), \mathrm{H}-3 \rightarrow \mathrm{L}+2(20 \%)$ \\
\hline 45 & 291.508025 & 0 & Triplet-A & \\
\hline 46 & 290.089361 & 0.0152 & Singlet-A & $\mathrm{H}-7 \rightarrow \mathrm{L}+1(32 \%), \mathrm{H}-2 \rightarrow \mathrm{L}+3(25 \%), \mathrm{H}-1 \rightarrow \mathrm{L}+3(10 \%)$ \\
\hline 47 & 289.22318 & 0 & Triplet-A & $\mathrm{H}-2 \rightarrow \mathrm{L}+3(10 \%)$ \\
\hline 48 & 288.301809 & 0 & Triplet-A & $\mathrm{HOMO} \rightarrow \mathrm{L}+10(19 \%)$ \\
\hline 49 & 287.166631 & 0.0053 & Singlet-A & $\mathrm{H}-8 \rightarrow \mathrm{LUMO}(40 \%), \mathrm{H}-7 \rightarrow \mathrm{L}+1(10 \%), \mathrm{HOMO} \rightarrow \mathrm{L}+4(12 \%)$ \\
\hline 50 & 286.708429 & 0 & Triplet-A & $\mathrm{H}-6 \rightarrow \mathrm{L}+1(48 \%), \mathrm{H}-1 \rightarrow \mathrm{L}+2(10 \%)$ \\
\hline 51 & 286.066757 & 0.0047 & Singlet-A & $\mathrm{H}-6 \rightarrow \mathrm{L}+1(60 \%)$ \\
\hline 52 & 285.6647 & 0.0007 & Singlet-A & $\mathrm{H}-2 \rightarrow \mathrm{L}+2(25 \%), \mathrm{H}-1 \rightarrow \mathrm{L}+2(48 \%)$ \\
\hline 53 & 285.217835 & 0.082 & Singlet-A & $\mathrm{H}-7 \rightarrow \mathrm{L}+1(26 \%), \mathrm{H}-2 \rightarrow \mathrm{L}+3(22 \%), \mathrm{HOMO} \rightarrow \mathrm{L}+4(14 \%)$ \\
\hline 54 & 285.027686 & 0.0429 & Singlet-A & $\mathrm{H}-9 \rightarrow \mathrm{LUMO}(32 \%), \mathrm{H}-8 \rightarrow \mathrm{LUMO}(23 \%), \mathrm{HOMO} \rightarrow \mathrm{L}+4(31 \%)$ \\
\hline 55 & 284.439177 & 0.0334 & Singlet-A & $\mathrm{H}-8 \rightarrow \mathrm{L}+1(10 \%), \mathrm{H}-4 \rightarrow \mathrm{L}+3(21 \%), \mathrm{H}-3 \rightarrow \mathrm{L}+3(22 \%), \mathrm{HOMO} \rightarrow \mathrm{L}+5(16 \%)$ \\
\hline 56 & 283.879091 & 0.0126 & Singlet-A & $\mathrm{H}-11 \rightarrow \mathrm{LUMO}(12 \%), \mathrm{H}-8 \rightarrow \mathrm{L}+1(15 \%), \mathrm{HOMO} \rightarrow \mathrm{L}+5(15 \%)$ \\
\hline 57 & 283.133576 & 0.0003 & Singlet-A & $\mathrm{H}-4 \rightarrow \mathrm{L}+2(23 \%), \mathrm{H}-3 \rightarrow \mathrm{L}+2(20 \%), \mathrm{H}-2 \rightarrow \mathrm{L}+3(10 \%), \mathrm{H}-1 \rightarrow \mathrm{L}+3(20 \%)$ \\
\hline 58 & 282.926824 & 0.035 & Singlet-A & $\mathrm{H}-11 \rightarrow$ LUMO $(19 \%), \mathrm{H}-9 \rightarrow$ LUMO $(12 \%), \mathrm{H}-8 \rightarrow \mathrm{L}+1(39 \%)$ \\
\hline 59 & 281.098676 & 0.0669 & Singlet-A & $\mathrm{H}-9 \rightarrow \mathrm{LUMO}(19 \%), \mathrm{H}-2 \rightarrow \mathrm{L}+4(10 \%)$ \\
\hline 60 & 279.621545 & 0.0146 & Singlet-A & $\mathrm{H}-2 \rightarrow \mathrm{L}+5(31 \%), \mathrm{H}-1 \rightarrow \mathrm{L}+5(10 \%)$ \\
\hline
\end{tabular}


Table S5. Energy levels and contributions of the lower lying transitions of $\operatorname{Ir}\left(\mathbf{p p y S C F} \mathbf{S C}_{3}\right.$ calculated from the TD-DFT calculation.

\begin{tabular}{|c|c|c|c|c|}
\hline No. & Wavelength (nm) & Osc. Strength & Symmetry & Major contributions \\
\hline 1 & 435.781495 & $\mathbf{0}$ & Triplet-A & HOMO $\rightarrow$ LUMO (55\%) \\
\hline 2 & 434.346446 & 0 & Triplet-A & $\mathrm{HOMO} \rightarrow \mathrm{L}+1(32 \%), \mathrm{HOMO} \rightarrow \mathrm{L}+2(17 \%)$ \\
\hline 3 & 434.33123 & 0 & Triplet-A & $\mathrm{HOMO} \rightarrow \mathrm{L}+1(17 \%), \mathrm{HOMO} \rightarrow \mathrm{L}+2(32 \%)$ \\
\hline 4 & 391.673331 & 0.0046 & Singlet-A & HOMO $\rightarrow$ LUMO $(97 \%)$ \\
\hline 5 & 391.40131 & 0 & Triplet-A & $\mathrm{H}-2 \rightarrow \mathrm{L}+2(36 \%), \mathrm{H}-1 \rightarrow \mathrm{L}+1(39 \%)$ \\
\hline 6 & 387.317463 & 0 & Triplet-A & $\mathrm{H}-2 \rightarrow \mathrm{L}+1(20 \%), \mathrm{H}-1 \rightarrow \mathrm{LUMO}(37 \%), \mathrm{H}-1 \rightarrow \mathrm{L}+2(20 \%)$ \\
\hline 7 & 387.160233 & 0 & Triplet-A & $\mathrm{H}-2 \rightarrow \mathrm{LUMO}(36 \%), \mathrm{H}-2 \rightarrow \mathrm{L}+2(22 \%), \mathrm{H}-1 \rightarrow \mathrm{L}+1(19 \%)$ \\
\hline 8 & 385.750888 & 0.0022 & Singlet-A & HOMO $\rightarrow \mathrm{L}+1(97 \%)$ \\
\hline 9 & 385.678891 & 0.0023 & Singlet-A & HОМO $\rightarrow \mathrm{L}+2(97 \%)$ \\
\hline 10 & 378.150465 & 0 & Triplet-A & $\mathrm{H}-2 \rightarrow \mathrm{L}+2(10 \%), \mathrm{H}-1 \rightarrow \mathrm{LUMO}(11 \%), \mathrm{H}-1 \rightarrow \mathrm{L}+1(11 \%), \mathrm{HOMO} \rightarrow \mathrm{L}+1(29 \%)$ \\
\hline 11 & 378.104337 & 0 & Triplet-A & $\mathrm{H}-2 \rightarrow \mathrm{LUMO}(11 \%), \mathrm{H}-2 \rightarrow \mathrm{L}+1(11 \%), \mathrm{H}-1 \rightarrow \mathrm{L}+2(10 \%), \mathrm{HOMO} \rightarrow \mathrm{L}+2(29 \%)$ \\
\hline 12 & 375.005121 & 0 & Triplet-A & $\mathrm{H}-2 \rightarrow \mathrm{L}+1(17 \%), \mathrm{H}-1 \rightarrow \mathrm{L}+2(18 \%), \mathrm{HOMO} \rightarrow \mathrm{LUMO}(35 \%)$ \\
\hline 13 & 366.405204 & 0.0288 & Singlet-A & $\mathrm{H}-1 \rightarrow \mathrm{LUMO}(86 \%)$ \\
\hline 14 & 366.307776 & 0.0282 & Singlet-A & $\mathrm{H}-2 \rightarrow \mathrm{LUMO}(86 \%)$ \\
\hline 15 & 364.241585 & 0.0028 & Singlet-A & $\mathrm{H}-2 \rightarrow \mathrm{L}+1(44 \%), \mathrm{H}-1 \rightarrow \mathrm{L}+2(46 \%)$ \\
\hline 16 & 362.442098 & 0 & Triplet-A & $\mathrm{H}-4 \rightarrow \mathrm{LUMO}(15 \%), \mathrm{H}-2 \rightarrow \mathrm{LUMO}(20 \%), \mathrm{H}-1 \rightarrow \mathrm{L}+1(10 \%)$ \\
\hline 17 & 362.410315 & 0 & Triplet-A & $\mathrm{H}-3 \rightarrow \mathrm{LUMO}(15 \%), \mathrm{H}-2 \rightarrow \mathrm{L}+1(10 \%), \mathrm{H}-1 \rightarrow \mathrm{LUMO}(19 \%), \mathrm{H}-1 \rightarrow \mathrm{L}+2(10 \%)$ \\
\hline 18 & 360.912273 & 0 & Triplet-A & $\mathrm{H}-5 \rightarrow \mathrm{LUMO}(10 \%), \mathrm{H}-2 \rightarrow \mathrm{L}+1(17 \%), \mathrm{H}-1 \rightarrow \mathrm{L}+2(18 \%)$ \\
\hline 19 & 356.460793 & 0 & Triplet-A & $\mathrm{HOMO} \rightarrow \mathrm{L}+3(61 \%)$ \\
\hline 20 & 356.255942 & 0.0574 & Singlet-A & $\mathrm{H}-2 \rightarrow \mathrm{L}+1(45 \%), \mathrm{H}-1 \rightarrow \mathrm{L}+2(44 \%)$ \\
\hline 21 & 356.163836 & 0.0585 & Singlet-A & $\mathrm{H}-2 \rightarrow \mathrm{L}+2(43 \%), \mathrm{H}-1 \rightarrow \mathrm{L}+1(45 \%)$ \\
\hline 22 & 352.96986 & 0 & Triplet-A & $\mathrm{H}-1 \rightarrow \mathrm{L}+3(45 \%), \mathrm{HOMO} \rightarrow \mathrm{L}+4(10 \%)$ \\
\hline 23 & 352.829235 & 0 & Triplet-A & $\mathrm{H}-2 \rightarrow \mathrm{L}+3(45 \%), \mathrm{HOMO} \rightarrow \mathrm{L}+5(10 \%)$ \\
\hline 24 & 348.123524 & 0.0112 & Singlet-A & $\mathrm{H}-2 \rightarrow \mathrm{L}+2(34 \%), \mathrm{H}-1 \rightarrow \mathrm{L}+1(32 \%), \mathrm{HOMO} \rightarrow \mathrm{L}+3(25 \%)$ \\
\hline 25 & 335.964104 & 0.0544 & Singlet-A & $\mathrm{H}-2 \rightarrow \mathrm{L}+2(11 \%), \mathrm{H}-1 \rightarrow \mathrm{L}+1(10 \%), \mathrm{HOMO} \rightarrow \mathrm{L}+3(71 \%)$ \\
\hline 26 & 331.987878 & 0 & Triplet-A & $\mathrm{H}-2 \rightarrow \mathrm{L}+5(15 \%), \mathrm{H}-1 \rightarrow \mathrm{L}+4(15 \%), \mathrm{HOMO} \rightarrow \mathrm{L}+3(24 \%), \mathrm{HOMO} \rightarrow \mathrm{L}+6(11 \%)$ \\
\hline 27 & 329.438536 & 0 & Triplet-A & $\mathrm{H}-1 \rightarrow \mathrm{L}+7(12 \%), \mathrm{HOMO} \rightarrow \mathrm{L}+6(21 \%)$ \\
\hline 28 & 329.036366 & 0 & Triplet-A & $\mathrm{H}-2 \rightarrow \mathrm{L}+8(10 \%), \mathrm{H}-1 \rightarrow \mathrm{L}+6(15 \%), \mathrm{HOMO} \rightarrow \mathrm{L}+4(10 \%), \mathrm{HOMO} \rightarrow \mathrm{L}+7(16 \%)$ \\
\hline 29 & 328.94034 & 0 & Triplet-A & $\mathrm{H}-2 \rightarrow \mathrm{L}+6(15 \%), \mathrm{HOMO} \rightarrow \mathrm{L}+5(11 \%), \mathrm{HOMO} \rightarrow \mathrm{L}+8(17 \%)$ \\
\hline 30 & 325.306832 & 0 & Triplet-A & $\mathrm{H}-1 \rightarrow \mathrm{L}+3(21 \%), \mathrm{HOMO} \rightarrow \mathrm{L}+4(49 \%)$ \\
\hline 31 & 325.178853 & 0 & Triplet-A & $\mathrm{H}-2 \rightarrow \mathrm{L}+3(21 \%), \mathrm{HOMO} \rightarrow \mathrm{L}+5(49 \%)$ \\
\hline 32 & 324.302773 & 0.0026 & Singlet-A & $\mathrm{H}-1 \rightarrow \mathrm{L}+3(92 \%)$ \\
\hline 33 & 324.184058 & 0.0026 & Singlet-A & $\mathrm{H}-2 \rightarrow \mathrm{L}+3(92 \%)$ \\
\hline 34 & 317.883735 & 0.0199 & Singlet-A & $\mathrm{HOMO} \rightarrow \mathrm{L}+4(90 \%)$ \\
\hline 35 & 317.834841 & 0.0198 & Singlet-A & $\mathrm{HOMO} \rightarrow \mathrm{L}+5(90 \%)$ \\
\hline 36 & 312.972846 & 0 & Triplet-A & $\mathrm{H}-2 \rightarrow \mathrm{L}+5(21 \%), \mathrm{H}-1 \rightarrow \mathrm{L}+3(15 \%), \mathrm{H}-1 \rightarrow \mathrm{L}+4(22 \%), \mathrm{HOMO} \rightarrow \mathrm{L}+4(20 \%)$ \\
\hline 37 & 312.917553 & 0 & Triplet-A & $\mathrm{H}-2 \rightarrow \mathrm{L}+3(15 \%), \mathrm{H}-2 \rightarrow \mathrm{L}+4(21 \%), \mathrm{H}-1 \rightarrow \mathrm{L}+5(22 \%), \mathrm{HOMO} \rightarrow \mathrm{L}+5(20 \%)$ \\
\hline 38 & 307.271854 & 0 & Triplet-A & $\mathrm{H}-2 \rightarrow \mathrm{L}+4(40 \%), \mathrm{H}-1 \rightarrow \mathrm{L}+5(40 \%)$ \\
\hline 39 & 304.651923 & 0.0007 & Singlet-A & $\mathrm{H}-2 \rightarrow \mathrm{L}+4(12 \%), \mathrm{H}-2 \rightarrow \mathrm{L}+5(23 \%), \mathrm{H}-1 \rightarrow \mathrm{L}+4(45 \%), \mathrm{H}-1 \rightarrow \mathrm{L}+5(17 \%)$ \\
\hline 40 & 304.233291 & 0.0103 & Singlet-A & $\mathrm{H}-2 \rightarrow \mathrm{L}+4(16 \%), \mathrm{H}-2 \rightarrow \mathrm{L}+5(20 \%), \mathrm{H}-1 \rightarrow \mathrm{L}+4(23 \%), \mathrm{H}-1 \rightarrow \mathrm{L}+5(37 \%)$ \\
\hline 41 & 304.218361 & 0.0105 & Singlet-A & $\mathrm{H}-2 \rightarrow \mathrm{L}+4(29 \%), \mathrm{H}-2 \rightarrow \mathrm{L}+5(32 \%), \mathrm{H}-1 \rightarrow \mathrm{L}+4(20 \%), \mathrm{H}-1 \rightarrow \mathrm{L}+5(16 \%)$ \\
\hline 42 & 303.860483 & 0.0031 & Singlet-A & $\mathrm{H}-2 \rightarrow \mathrm{L}+4(38 \%), \mathrm{H}-2 \rightarrow \mathrm{L}+5(21 \%), \mathrm{H}-1 \rightarrow \mathrm{L}+5(25 \%)$ \\
\hline 43 & 299.146342 & 0 & Triplet-A & $\mathrm{H}-5 \rightarrow \mathrm{L}+3(16 \%), \mathrm{H}-1 \rightarrow \mathrm{L}+4(10 \%), \mathrm{HOMO} \rightarrow \mathrm{L}+6(13 \%)$ \\
\hline 44 & 298.973217 & 0 & Triplet-A & $\mathrm{H}-4 \rightarrow \mathrm{L}+3(20 \%)$ \\
\hline 45 & 298.944382 & 0 & Triplet-A & $\mathrm{H}-3 \rightarrow \mathrm{L}+3(19 \%)$ \\
\hline 46 & 289.135498 & 0 & Triplet-A & \\
\hline 47 & 289.088307 & 0 & Triplet-A & $\mathrm{H}-8 \rightarrow \mathrm{LUMO}(10 \%), \mathrm{H}-8 \rightarrow \mathrm{L}+1(10 \%)$ \\
\hline 48 & 289.034393 & 0 & Triplet-A & \\
\hline 49 & 288.221385 & 0.0205 & Singlet-A & $\mathrm{H}-3 \rightarrow \mathrm{LUMO}(58 \%), \mathrm{HOMO} \rightarrow \mathrm{L}+7(17 \%)$ \\
\hline 50 & 288.147702 & 0.0207 & Singlet-A & $\mathrm{H}-4 \rightarrow \mathrm{LUMO}(57 \%), \mathrm{HOMO} \rightarrow \mathrm{L}+8(17 \%)$ \\
\hline 51 & 285.139122 & 0.0067 & Singlet-A & $\mathrm{H}-4 \rightarrow \mathrm{L}+2(17 \%), \mathrm{H}-3 \rightarrow \mathrm{L}+1(19 \%), \mathrm{HOMO} \rightarrow \mathrm{L}+6(35 \%)$ \\
\hline 52 & 284.406554 & 0.02 & Singlet-A & $\begin{array}{l}\mathrm{H}-4 \rightarrow \mathrm{L}+2(11 \%), \mathrm{H}-3 \rightarrow \mathrm{LUMO}(19 \%), \mathrm{H}-3 \rightarrow \mathrm{L}+1(10 \%), \mathrm{HOMO} \rightarrow \mathrm{L}+7(24 \%), \\
\mathrm{HOMO} \rightarrow \mathrm{L}+8(14 \%)\end{array}$ \\
\hline 53 & 284.354371 & 0.0188 & Singlet-A & $\begin{array}{l}\mathrm{H}-4 \rightarrow \mathrm{LUMO}(19 \%), \mathrm{H}-4 \rightarrow \mathrm{L}+1(11 \%), \mathrm{H}-3 \rightarrow \mathrm{L}+2(10 \%), \mathrm{HOMO} \rightarrow \mathrm{L}+7(14 \%), \\
\mathrm{HOMO} \rightarrow \mathrm{L}+8(24 \%)\end{array}$ \\
\hline 54 & 283.334155 & 0.0074 & Singlet-A & $\mathrm{H}-4 \rightarrow \mathrm{L}+2(11 \%), \mathrm{H}-3 \rightarrow \mathrm{L}+1(38 \%), \mathrm{HOMO} \rightarrow \mathrm{L}+6(21 \%)$ \\
\hline 55 & 283.049547 & 0.0258 & Singlet-A & $\mathrm{H}-4 \rightarrow \mathrm{L}+2(29 \%), \mathrm{H}-2 \rightarrow \mathrm{L}+6(10 \%), \mathrm{H}-1 \rightarrow \mathrm{L}+6(13 \%), \mathrm{HOMO} \rightarrow \mathrm{L}+7(26 \%)$ \\
\hline 56 & 281.903988 & 0.0269 & Singlet-A & $\mathrm{H}-4 \rightarrow \mathrm{L}+1(18 \%), \mathrm{H}-3 \rightarrow \mathrm{L}+2(11 \%), \mathrm{H}-2 \rightarrow \mathrm{L}+6(16 \%), \mathrm{HOMO} \rightarrow \mathrm{L}+8(27 \%)$ \\
\hline 57 & 281.846313 & 0.0025 & Singlet-A & $\mathrm{H}-5 \rightarrow \mathrm{LUMO}(34 \%), \mathrm{H}-4 \rightarrow \mathrm{L}+1(18 \%), \mathrm{H}-3 \rightarrow \mathrm{L}+2(20 \%), \mathrm{HOMO} \rightarrow \mathrm{L}+6(16 \%)$ \\
\hline 58 & 281.801471 & 0.1922 & Singlet-A & $\mathrm{H}-5 \rightarrow \mathrm{LUMO}(47 \%), \mathrm{H}-4 \rightarrow \mathrm{L}+1(10 \%), \mathrm{H}-3 \rightarrow \mathrm{L}+2(11 \%)$ \\
\hline 59 & 281.277236 & 0.0188 & Singlet-A & $\mathrm{H}-5 \rightarrow \mathrm{L}+1(73 \%)$ \\
\hline 60 & 279.174513 & 0.0194 & Singlet-A & $\mathrm{H}-5 \rightarrow \mathrm{L}+2(74 \%)$ \\
\hline
\end{tabular}


Table S6. Energy levels and contributions of the lower lying transitions of $\operatorname{Ir}(\mathbf{p p y S F})_{3}$ calculated from the TD-DFT calculation.

\begin{tabular}{|c|c|c|c|c|}
\hline No. & Wavelength (nm) & Osc. Strength & Symmetry & Major contributions \\
\hline 1 & 437.099724 & $\mathbf{0}$ & Triplet-A & $\mathrm{H}-1 \rightarrow \mathrm{L}+2(-10 \%)$, HOMO $\rightarrow$ LUMO $(54 \%)$ \\
\hline 2 & 435.855082 & 0 & Triplet-A & $\mathrm{H}-2 \rightarrow \mathrm{LUMO}(12 \%), \mathrm{HOMO} \rightarrow \mathrm{L}+1(48 \%)$ \\
\hline 3 & 435.640677 & 0 & Triplet-A & $\mathrm{H}-1 \rightarrow \mathrm{LUMO}(-13 \%), \mathrm{HOMO} \rightarrow \mathrm{L}+2(47 \%)$ \\
\hline 4 & 392.949216 & 0 & Triplet-A & $\mathrm{H}-2 \rightarrow \mathrm{L}+1(11 \%), \mathrm{H}-2 \rightarrow \mathrm{L}+2(32 \%), \mathrm{H}-1 \rightarrow \mathrm{L}+1(31 \%)$ \\
\hline 5 & 392.414422 & 0.0048 & Singlet-A & HOMO $\rightarrow$ LUMO $(97 \%)$ \\
\hline 6 & 388.991738 & 0 & Triplet-A & $\mathrm{H}-2 \rightarrow \mathrm{LUMO}(42 \%), \mathrm{H}-2 \rightarrow \mathrm{L}+2(13 \%), \mathrm{H}-1 \rightarrow \mathrm{L}+1(-13 \%)$ \\
\hline 7 & 388.540698 & 0 & Triplet-A & $\mathrm{H}-2 \rightarrow \mathrm{L}+1(-14 \%), \mathrm{H}-1 \rightarrow \mathrm{LUMO}(38 \%), \mathrm{H}-1 \rightarrow \mathrm{L}+2(-16 \%)$ \\
\hline 8 & 386.831414 & 0.0023 & Singlet-A & HOMO $\rightarrow \mathrm{L}+1(97 \%)$ \\
\hline 9 & 386.337208 & 0.0025 & Singlet-A & HOМO $\rightarrow \mathrm{L}+2(96 \%)$ \\
\hline 10 & 379.211918 & 0 & Triplet-A & $\mathrm{H}-1 \rightarrow \mathrm{LUMO}(18 \%), \mathrm{HOMO} \rightarrow \mathrm{L}+2(34 \%)$ \\
\hline 11 & 378.899018 & 0 & Triplet-A & $\mathrm{H}-2 \rightarrow \mathrm{LUMO}(-15 \%), \mathrm{HOMO} \rightarrow \mathrm{L}+1(32 \%)$ \\
\hline 12 & 375.957719 & 0 & Triplet-A & $\mathrm{H}-2 \rightarrow \mathrm{L}+1(-16 \%), \mathrm{H}-1 \rightarrow \mathrm{L}+2(14 \%), \mathrm{HOMO} \rightarrow \mathrm{LUMO}(34 \%)$ \\
\hline 13 & 367.979511 & 0.0258 & Singlet-A & $\mathrm{H}-1 \rightarrow \mathrm{LUMO}(87 \%)$ \\
\hline 14 & 367.477805 & 0.0291 & Singlet-A & $\mathrm{H}-2 \rightarrow \mathrm{LUMO}(87 \%)$ \\
\hline 15 & 365.700194 & 0.0029 & Singlet-A & $\mathrm{H}-2 \rightarrow \mathrm{L}+1(42 \%), \mathrm{H}-2 \rightarrow \mathrm{L}+2(-10 \%), \mathrm{H}-1 \rightarrow \mathrm{L}+1(-10 \%), \mathrm{H}-1 \rightarrow \mathrm{L}+2(-35 \%)$ \\
\hline 16 & 363.502218 & 0 & Triplet-A & $\mathrm{H}-4 \rightarrow$ LUMO $(-16 \%), \mathrm{H}-1 \rightarrow$ LUMO $(21 \%)$ \\
\hline 17 & 363.342428 & 0 & Triplet-A & $\mathrm{H}-3 \rightarrow$ LUMO $(-15 \%), \mathrm{H}-2 \rightarrow$ LUMO $(21 \%)$ \\
\hline 18 & 361.71 & 0 & Triplet-A & $\mathrm{H}-5 \rightarrow \mathrm{LUMO}(-11 \%), \mathrm{H}-2 \rightarrow \mathrm{L}+1(-13 \%), \mathrm{H}-1 \rightarrow \mathrm{L}+2(14 \%)$ \\
\hline 19 & 357.743996 & 0.0556 & Singlet-A & $\mathrm{H}-2 \rightarrow \mathrm{L}+2(-36 \%), \mathrm{H}-1 \rightarrow \mathrm{L}+1(40 \%)$ \\
\hline 20 & 357.568601 & 0 & Triplet-A & $\mathrm{HOMO} \rightarrow \mathrm{L}+3(63 \%)$ \\
\hline 21 & 357.455201 & 0.0589 & Singlet-A & $\mathrm{H}-2 \rightarrow \mathrm{L}+1(36 \%), \mathrm{H}-1 \rightarrow \mathrm{L}+2(40 \%)$ \\
\hline 22 & 354.258348 & 0 & Triplet-A & $\mathrm{H}-1 \rightarrow \mathrm{L}+3(43 \%)$ \\
\hline 23 & 354.086353 & 0 & Triplet-A & $\mathrm{H}-2 \rightarrow \mathrm{L}+3(43 \%)$ \\
\hline 24 & 349.386622 & 0.0121 & Singlet-A & $\mathrm{H}-2 \rightarrow \mathrm{L}+2(31 \%), \mathrm{H}-1 \rightarrow \mathrm{L}+1(28 \%), \mathrm{HOMO} \rightarrow \mathrm{L}+3(-23 \%)$ \\
\hline 25 & 336.545431 & 0.0506 & Singlet-A & $\mathrm{HOMO} \rightarrow \mathrm{L}+3(73 \%)$ \\
\hline 26 & 333.207925 & 0 & Triplet-A & $\mathrm{H}-2 \rightarrow \mathrm{L}+4(-11 \%), \mathrm{H}-1 \rightarrow \mathrm{L}+5(11 \%), \mathrm{HOMO} \rightarrow \mathrm{L}+3(24 \%), \mathrm{HOMO} \rightarrow \mathrm{L}+6(15 \%)$ \\
\hline 27 & 330.22596 & 0 & Triplet-A & $\mathrm{H}-1 \rightarrow \mathrm{L}+5(-10 \%), \mathrm{H}-1 \rightarrow \mathrm{L}+8(11 \%), \mathrm{HOMO} \rightarrow \mathrm{L}+6(16 \%)$ \\
\hline 28 & 330.041358 & 0 & Triplet-A & $\mathrm{H}-2 \rightarrow \mathrm{L}+6(18 \%), \mathrm{HOMO} \rightarrow \mathrm{L}+7(21 \%)$ \\
\hline 29 & 329.97987 & 0 & Triplet-A & $\mathrm{H}-1 \rightarrow \mathrm{L}+6(14 \%), \mathrm{HOMO} \rightarrow \mathrm{L}+8(21 \%)$ \\
\hline 30 & 326.314875 & 0 & Triplet-A & $\mathrm{H}-2 \rightarrow \mathrm{L}+3(-22 \%), \mathrm{HOMO} \rightarrow \mathrm{L}+4(45 \%)$ \\
\hline 31 & 326.108884 & 0 & Triplet-A & $\mathrm{H}-1 \rightarrow \mathrm{L}+3(22 \%), \mathrm{HOMO} \rightarrow \mathrm{L}+5(45 \%)$ \\
\hline 32 & 325.321657 & 0.0023 & Singlet-A & $\mathrm{H}-1 \rightarrow \mathrm{L}+3(93 \%)$ \\
\hline 33 & 325.236318 & 0.0023 & Singlet-A & $\mathrm{H}-2 \rightarrow \mathrm{L}+3(93 \%)$ \\
\hline 34 & 318.534894 & 0.0197 & Singlet-A & $\mathrm{HOMO} \rightarrow \mathrm{L}+4(91 \%)$ \\
\hline 35 & 318.297742 & 0.0203 & Singlet-A & $\mathrm{HOMO} \rightarrow \mathrm{L}+5(91 \%)$ \\
\hline 36 & 313.969299 & 0 & Triplet-A & $\mathrm{H}-2 \rightarrow \mathrm{L}+5(-13 \%), \mathrm{H}-1 \rightarrow \mathrm{L}+3(14 \%), \mathrm{H}-1 \rightarrow \mathrm{L}+4(15 \%), \mathrm{HOMO} \rightarrow \mathrm{L}+4(20 \%)$ \\
\hline 37 & 313.596056 & 0 & Triplet-A & $\mathrm{H}-2 \rightarrow \mathrm{L}+3(14 \%), \mathrm{H}-2 \rightarrow \mathrm{L}+4(-14 \%), \mathrm{H}-1 \rightarrow \mathrm{L}+5(-15 \%), \mathrm{HOMO} \rightarrow \mathrm{L}+5(19 \%)$ \\
\hline 38 & 308.232241 & 0 & Triplet-A & $\mathrm{H}-2 \rightarrow \mathrm{L}+4(10 \%), \mathrm{H}-2 \rightarrow \mathrm{L}+5(37 \%), \mathrm{H}-1 \rightarrow \mathrm{L}+4(34 \%), \mathrm{H}-1 \rightarrow \mathrm{L}+5(-10 \%)$ \\
\hline 39 & 305.739141 & 0.0012 & Singlet-A & $\mathrm{H}-2 \rightarrow \mathrm{L}+4(-30 \%), \mathrm{H}-1 \rightarrow \mathrm{L}+4(40 \%), \mathrm{H}-1 \rightarrow \mathrm{L}+5(18 \%)$ \\
\hline 40 & 305.287444 & 0.0099 & Singlet-A & $\mathrm{H}-2 \rightarrow \mathrm{L}+4(40 \%), \mathrm{H}-2 \rightarrow \mathrm{L}+5(-17 \%), \mathrm{H}-1 \rightarrow \mathrm{L}+4(31 \%)$ \\
\hline 41 & 305.21229 & 0.0091 & Singlet-A & $\mathrm{H}-2 \rightarrow \mathrm{L}+4(12 \%), \mathrm{H}-2 \rightarrow \mathrm{L}+5(24 \%), \mathrm{H}-1 \rightarrow \mathrm{L}+4(-15 \%), \mathrm{H}-1 \rightarrow \mathrm{L}+5(46 \%)$ \\
\hline 42 & 304.739675 & 0.0037 & Singlet-A & $\mathrm{H}-2 \rightarrow \mathrm{L}+4(13 \%), \mathrm{H}-2 \rightarrow \mathrm{L}+5(46 \%), \mathrm{H}-1 \rightarrow \mathrm{L}+4(10 \%), \mathrm{H}-1 \rightarrow \mathrm{L}+5(-23 \%)$ \\
\hline 43 & 299.585204 & 0 & Triplet-A & $\mathrm{H}-5 \rightarrow \mathrm{L}+3(14 \%), \mathrm{H}-2 \rightarrow \mathrm{L}+4(10 \%), \mathrm{H}-1 \rightarrow \mathrm{L}+4(-10 \%), \mathrm{HOMO} \rightarrow \mathrm{L}+6(10 \%)$ \\
\hline 44 & 299.4188 & 0 & Triplet-A & $\mathrm{H}-4 \rightarrow \mathrm{L}+3(15 \%), \mathrm{H}-2 \rightarrow \mathrm{L}+5(15 \%)$ \\
\hline 45 & 299.295924 & 0 & Triplet-A & $\mathrm{H}-3 \rightarrow \mathrm{L}+3(18 \%), \mathrm{H}-1 \rightarrow \mathrm{L}+5(12 \%)$ \\
\hline 46 & 289.443998 & 0 & Triplet-A & $\mathrm{H}-7 \rightarrow \mathrm{L}+1(-13 \%), \mathrm{H}-3 \rightarrow \mathrm{L}+1(10 \%)$ \\
\hline 47 & 289.423728 & 0 & Triplet-A & $\mathrm{H}-8 \rightarrow \mathrm{L}+2(11 \%)$ \\
\hline 48 & 289.248172 & 0 & Triplet-A & \\
\hline 49 & 288.266302 & 0.0245 & Singlet-A & $\mathrm{H}-4 \rightarrow \mathrm{LUMO}(33 \%), \mathrm{H}-3 \rightarrow \mathrm{LUMO}(-25 \%), \mathrm{HOMO} \rightarrow \mathrm{L}+7(-27 \%)$ \\
\hline 50 & 288.065373 & 0.0253 & Singlet-A & $\mathrm{H}-4 \rightarrow$ LUMO $(25 \%), \mathrm{H}-3 \rightarrow$ LUMO $(34 \%), \mathrm{HOMO} \rightarrow \mathrm{L}+8(25 \%)$ \\
\hline 51 & 285.439121 & 0.0067 & Singlet-A & $\mathrm{H}-4 \rightarrow \mathrm{L}+2(-18 \%), \mathrm{H}-3 \rightarrow \mathrm{L}+1(23 \%), \mathrm{HOMO} \rightarrow \mathrm{L}+6(46 \%)$ \\
\hline 52 & 285.399698 & 0.0178 & Singlet-A & $\mathrm{H}-4 \rightarrow \mathrm{L}+1(13 \%), \mathrm{H}-3 \rightarrow \mathrm{LUMO}(-24 \%), \mathrm{H}-3 \rightarrow \mathrm{L}+2(-11 \%), \mathrm{HOMO} \rightarrow \mathrm{L}+7(37 \%)$ \\
\hline 53 & 285.366853 & 0.0139 & Singlet-A & $\mathrm{H}-4 \rightarrow \mathrm{LUMO}(-25 \%), \mathrm{H}-4 \rightarrow \mathrm{L}+2(10 \%), \mathrm{H}-3 \rightarrow \mathrm{L}+1(15 \%), \mathrm{HOMO} \rightarrow \mathrm{L}+8(34 \%)$ \\
\hline 54 & 284.705007 & 0.0188 & Singlet-A & $\mathrm{H}-4 \rightarrow \mathrm{L}+1(13 \%), \mathrm{H}-3 \rightarrow \mathrm{L}+2(-17 \%), \mathrm{H}-2 \rightarrow \mathrm{L}+6(29 \%), \mathrm{HOMO} \rightarrow \mathrm{L}+7(-16 \%)$ \\
\hline 55 & 284.404589 & 0.0124 & Singlet-A & $\mathrm{H}-4 \rightarrow \mathrm{L}+2(40 \%), \mathrm{H}-1 \rightarrow \mathrm{L}+6(18 \%)$ \\
\hline 56 & 282.35143 & 0.011 & Singlet-A & $\begin{array}{l}\mathrm{H}-4 \rightarrow \mathrm{L}+1 \quad(20 \%), \mathrm{H}-3 \rightarrow \mathrm{L}+1(22 \%), \mathrm{H}-1 \rightarrow \mathrm{L}+6(10 \%), \mathrm{HOMO} \rightarrow \mathrm{L}+6 \quad(-13 \%) \\
\mathrm{HOMO} \rightarrow \mathrm{L}+8(-14 \%)\end{array}$ \\
\hline 57 & 282.261438 & 0.009 & Singlet-A & $\mathrm{H}-5 \rightarrow \mathrm{LUMO}(29 \%), \mathrm{H}-4 \rightarrow \mathrm{L}+1(-23 \%), \mathrm{H}-3 \rightarrow \mathrm{L}+2(-32 \%)$ \\
\hline 58 & 282.081625 & 0.1663 & Singlet-A & $\mathrm{H}-5 \rightarrow \mathrm{LUMO}(52 \%)$ \\
\hline 59 & 282.075207 & 0.0224 & Singlet-A & $\mathrm{H}-5 \rightarrow \mathrm{L}+1(51 \%), \mathrm{H}-5 \rightarrow \mathrm{L}+2(19 \%)$ \\
\hline 60 & 281.626696 & 0.0243 & Singlet-A & $\mathrm{H}-5 \rightarrow \mathrm{L}+1(-20 \%), \mathrm{H}-5 \rightarrow \mathrm{L}+2(50 \%)$ \\
\hline
\end{tabular}


Table S7. Crystal data and structure refinement for $\operatorname{Ir}\left(\operatorname{ppySCF}_{3}\right)_{2}(\mathbf{a c a c}), \operatorname{Ir}\left(\mathbf{p p y S C F}_{3}\right)_{2}(\mathbf{p i c})$, and $\operatorname{Ir}\left(\text { ppySCF}_{3}\right)_{2}$ (bor).

\begin{tabular}{|c|c|c|c|c|}
\hline Identification code & $\operatorname{Ir}\left(\text { ppySCF}_{3}\right)_{2}($ acac $)$ & $\operatorname{Ir}\left(\operatorname{ppySCF}_{3}\right)_{2}($ pic $)$ & \multicolumn{2}{|c|}{$\operatorname{Ir}\left(\operatorname{ppySCF}_{3}\right)_{2}($ bor $)$} \\
\hline Empirical formula & $\mathrm{C}_{29} \mathrm{H}_{21} \mathrm{~F}_{6} \operatorname{Ir~} \mathrm{N}_{2} \mathrm{~S}_{2} \mathrm{O}_{6}$ & $\mathrm{C}_{30} \mathrm{H}_{18} \mathrm{~F}_{6}$ Ir $\mathrm{N}_{3} \mathrm{O}_{6} \mathrm{~S}_{2}$ & \\
\hline Formula weight & 863.80 & 886.79 & \multicolumn{2}{|c|}{$\begin{array}{l}\mathrm{C}_{36} \mathrm{H}_{26} \mathrm{~B} \mathrm{~F}_{6} \text { Ir } \mathrm{N}_{10} \mathrm{~S}_{2} \mathrm{O}_{4} \\
1043.80\end{array}$} \\
\hline Temperature & $293(2) \mathrm{K}$ & 293(2) K & \multicolumn{2}{|l|}{ 293(2) K } \\
\hline Wavelength & $0.71073 \AA$ & $0.71073 \AA$ & \multicolumn{2}{|l|}{$0.71073 \AA$} \\
\hline Crystal system, space group & Orthorhombic, Pccn & Trigonal, $P-3$ & \multicolumn{2}{|l|}{ Triclinic, $P-1$} \\
\hline \multirow[t]{3}{*}{ Unit cell dimensions } & $a=24.426(4) \AA$ & $a=24.350(2) \AA$ & $a=12.250(4) \AA$ & $\alpha=103.579(5)^{\circ}$ \\
\hline & $b=7.646(1) \AA$ & $b=24.350(2) \AA$ & $b=12.533(4) \AA$ & $\beta=96.945(6)^{\circ}$ \\
\hline & $c=18.063(3) \AA$ & $c=10.845(8) \AA$ & $c=16.864(5) \AA$ & $\gamma=106.551(6)^{\circ}$ \\
\hline Volume & $3373.3(1) \AA^{3}$ & $5568.9(9) \AA^{3}$ & $2363.0(1) \AA^{3}$ & \\
\hline$Z$, Calculated density & $4,1.701 \mathrm{Mg} / \mathrm{m}^{3}$ & $6,1.587 \mathrm{Mg} / \mathrm{m}^{3}$ & $2,1.467 \mathrm{Mg} / \mathrm{m}^{3}$ & \\
\hline Absorption coefficient, $\mu$ & $4.157 \mathrm{~mm}^{-1}$ & $3.780 \mathrm{~mm}^{-1}$ & $2.982 \mathrm{~mm}^{-1}$ & \\
\hline$F(000)$ & 1680 & 2580 & 1024 & \\
\hline Crystal size & $0.35 \times 0.32 \times 0.25 \mathrm{~mm}$ & $0.20 \times 0.10 \times 0.10 \mathrm{~mm}$ & $0.40 \times 0.30 \times 0.10$ & \\
\hline$\theta$ range for data collection & 2.255 to $28.342^{\circ}$ & 2.112 to $28.371^{\circ}$ & 1.268 to $28.458^{\circ}$ & \\
\hline Limiting indices & $-32 \leq h \leq 32,-10 \leq k \leq 10,-24 \leq l \leq 24$ & $-32 \leq h \leq 32,-32 \leq k \leq 32,-14 \leq l \leq 14$ & $-16 \leq h \leq 16,-16$ & $k \leq 16,-22 \leq l \leq 22$ \\
\hline Reflections collected / unique & $42022 / 4205\left[R_{\text {int }}=0.0620\right]$ & $166273 / 9289\left[R_{\text {int }}=0.3268\right]$ & $20175 / 7755\left[R_{\text {int }}\right.$ & $0.0391]$ \\
\hline Completeness to $\theta=25.242$ & $99.9 \%$ & $99.9 \%$ & $99.8 \%$ & \\
\hline Refinement method & Full-matrix least-squares on $F^{2}$ & Full-matrix least-squares on $F^{2}$ & Full-matrix least- & uares on $F^{2}$ \\
\hline Data / restraints / parameters & $4205 / 0 / 209$ & $9289 / 0 / 433$ & $7755 / 0 / 541$ & \\
\hline Goodness-of-fit on $F^{2}$ & 0.700 & 1.171 & 0.733 & \\
\hline Final $R$ indices $[I>2 \sigma(I)]$ & $R_{1}^{a}=0.0602, w R_{2}^{b}=0.1708$ & $R_{1}^{a}=0.1229, w R_{2}^{b}=0.2484$ & $R_{1}^{a}=0.0348, w R_{2}$ & $=0.0990$ \\
\hline$R$ indices (all data) & $R_{1}=0.0719, w R_{2}=0.1854$ & $R_{1}=0.1895, w R_{2}=0.2765$ & $R_{1}=0.0453, w R_{2}$ & 0.1080 \\
\hline Largest diff. peak and hole & 3.779 and -5.999 e. $\AA^{-3}$ & 1.774 and -4.629 e. $\AA^{-3}$ & 0.886 and -0.415 & $\AA^{-3}$ \\
\hline
\end{tabular}


Table S8. Bond lengths $[\AA]$ and angles $\left[{ }^{\circ}\right]$ for $\operatorname{Ir}(\mathbf{p p y S C F} 3)_{2}$ (acac).

\begin{tabular}{|c|c|c|c|}
\hline \multicolumn{4}{|c|}{ Bond lengths $[\AA]$} \\
\hline $\operatorname{Ir}(1)-C(1)$ & $1.991(7)$ & $\mathrm{C}(1)-\mathrm{C}(6)$ & $1.400(1)$ \\
\hline $\operatorname{Ir}(1)-\mathrm{N}(1)$ & $2.047(7)$ & $C(1)-C(2)$ & $1.407(1)$ \\
\hline $\operatorname{Ir}(1)-\mathrm{O}(3)$ & $2.140(5)$ & $C(2)-C(3)$ & $1.379(1)$ \\
\hline $\mathrm{S}(1)-\mathrm{O}(1)$ & $1.421(8)$ & $C(3)-C(4)$ & $1.390(1)$ \\
\hline $\mathrm{S}(1)-\mathrm{O}(2)$ & $1.432(9)$ & $\mathrm{C}(4)-\mathrm{C}(5)$ & $1.411(1)$ \\
\hline $\mathrm{S}(1)-\mathrm{C}(4)$ & $1.743(8)$ & $C(5)-C(6)$ & $1.391(1)$ \\
\hline$S(1)-C(12)$ & $1.877(2)$ & $C(6)-C(7)$ & $1.484(1)$ \\
\hline $\mathrm{O}(3)-\mathrm{C}(13)$ & $1.255(1)$ & $\mathrm{C}(7)-\mathrm{C}(8)$ & $1.372(1)$ \\
\hline $\mathrm{F}(1)-\mathrm{C}(12)$ & $1.270(2)$ & $\mathrm{C}(8)-\mathrm{C}(9)$ & $1.397(1)$ \\
\hline $\mathrm{F}(2)-\mathrm{C}(12)$ & $1.322(2)$ & $\mathrm{C}(9)-\mathrm{C}(10)$ & $1.388(2)$ \\
\hline $\mathrm{F}(3)-\mathrm{C}(12)$ & $1.300(2)$ & $\mathrm{C}(10)-\mathrm{C}(11)$ & $1.345(1)$ \\
\hline $\mathrm{N}(1)-\mathrm{C}(11)$ & $1.345(1)$ & $C(13)-C(14)$ & $1.340(9)$ \\
\hline $\mathrm{N}(1)-\mathrm{C}(7)$ & $1.363(9)$ & $\mathrm{C}(13)-\mathrm{C}(15)$ & $1.507(1)$ \\
\hline \multicolumn{4}{|c|}{ Bond angles $\left[^{\circ}\right]$} \\
\hline $\mathrm{C}(1) \# 1-\operatorname{Ir}(1)-\mathrm{C}(1)$ & $92.3(4)$ & $\mathrm{C}(2)-\mathrm{C}(3)-\mathrm{C}(4)$ & $119.7(8)$ \\
\hline $\mathrm{C}(1) \# 1-\operatorname{Ir}(1)-\mathrm{N}(1)$ & $96.4(3)$ & $C(3)-C(4)-C(5)$ & $121.2(7)$ \\
\hline $\mathrm{C}(1)-\operatorname{Ir}(1)-\mathrm{N}(1)$ & $81.0(3)$ & $\mathrm{C}(3)-\mathrm{C}(4)-\mathrm{S}(1)$ & $120.1(7)$ \\
\hline $\mathrm{N}(1)-\operatorname{Ir}(1)-\mathrm{N}(1) \# 1$ & $176.4(3)$ & $C(5)-C(4)-S(1)$ & $118.7(6)$ \\
\hline $\mathrm{C}(1) \# 1-\operatorname{Ir}(1)-\mathrm{O}(3) \# 1$ & $90.2(3)$ & $C(6)-C(5)-C(4)$ & $117.2(7)$ \\
\hline $\mathrm{C}(1)-\operatorname{Ir}(1)-\mathrm{O}(3) \# 1$ & $174.2(3)$ & $C(5)-C(6)-C(1)$ & $123.3(7)$ \\
\hline $\mathrm{N}(1)-\operatorname{Ir}(1)-\mathrm{O}(3)$ & $93.5(2)$ & $C(5)-C(6)-C(7)$ & $121.0(7)$ \\
\hline $\mathrm{N}(1) \# 1-\operatorname{Ir}(1)-\mathrm{O}(3) \# 1$ & $89.1(2)$ & $C(1)-C(6)-C(7)$ & $115.8(7)$ \\
\hline $\mathrm{C}(1) \# 1-\operatorname{Ir}(1)-\mathrm{O}(3)$ & $174.2(3)$ & $\mathrm{N}(1)-\mathrm{C}(7)-\mathrm{C}(8)$ & $120.6(7)$ \\
\hline $\mathrm{C}(1)-\operatorname{Ir}(1)-\mathrm{O}(3)$ & $90.2(3)$ & $N(1)-C(7)-C(6)$ & $112.8(7)$ \\
\hline $\mathrm{N}(1)-\operatorname{Ir}(1)-\mathrm{O}(3)$ & $89.1(2)$ & $C(8)-C(7)-C(6)$ & $126.6(8)$ \\
\hline $\mathrm{O}(3) \# 1-\operatorname{Ir}(1)-\mathrm{O}(3)$ & $87.7(3)$ & $\mathrm{C}(7)-\mathrm{C}(8)-\mathrm{C}(9)$ & $119.9(8)$ \\
\hline $\mathrm{O}(1)-\mathrm{S}(1)-\mathrm{O}(2)$ & $119.4(7)$ & $\mathrm{C}(10)-\mathrm{C}(9)-\mathrm{C}(8)$ & 118.1(9) \\
\hline $\mathrm{O}(1)-\mathrm{S}(1)-\mathrm{C}(4)$ & $112.0(5)$ & $\mathrm{C}(11)-\mathrm{C}(10)-\mathrm{C}(9)$ & 119.6(9) \\
\hline $\mathrm{O}(2)-\mathrm{S}(1)-\mathrm{C}(4)$ & $109.8(5)$ & $\mathrm{C}(10)-\mathrm{C}(11)-\mathrm{N}(1)$ & $122.8(8)$ \\
\hline $\mathrm{O}(1)-\mathrm{S}(1)-\mathrm{C}(12)$ & $104.0(7)$ & $\mathrm{F}(1)-\mathrm{C}(12)-\mathrm{F}(3)$ & $113.0(2)$ \\
\hline $\mathrm{O}(2)-\mathrm{S}(1)-\mathrm{C}(12)$ & $108.8(9)$ & $\mathrm{F}(1)-\mathrm{C}(12)-\mathrm{F}(2)$ & $110.8(2)$ \\
\hline $\mathrm{C}(4)-\mathrm{S}(1)-\mathrm{C}(12)$ & $101.0(6)$ & $\mathrm{F}(3)-\mathrm{C}(12)-\mathrm{F}(2)$ & $107.4(1)$ \\
\hline $\mathrm{C}(13)-\mathrm{O}(3)-\operatorname{Ir}(1)$ & $125.7(5)$ & $\mathrm{F}(1)-\mathrm{C}(12)-\mathrm{S}(1)$ & $110.8(1)$ \\
\hline $\mathrm{C}(11)-\mathrm{N}(1)-\mathrm{C}(7)$ & $119.0(7)$ & $\mathrm{F}(3)-\mathrm{C}(12)-\mathrm{S}(1)$ & $108.1(1)$ \\
\hline $\mathrm{C}(11)-\mathrm{N}(1)-\operatorname{Ir}(1)$ & $125.3(5)$ & $\mathrm{F}(2)-\mathrm{C}(12)-\mathrm{S}(1)$ & $106.4(1)$ \\
\hline $\mathrm{C}(7)-\mathrm{N}(1)-\operatorname{Ir}(1)$ & $115.6(5)$ & $\mathrm{O}(3)-\mathrm{C}(13)-\mathrm{C}(14)$ & $121.2(7)$ \\
\hline$C(6)-C(1)-C(2)$ & $117.0(6)$ & $\mathrm{O}(3)-\mathrm{C}(13)-\mathrm{C}(15)$ & $115.2(9)$ \\
\hline$C(6)-C(1)-\operatorname{Ir}(1)$ & $114.1(5)$ & $\mathrm{C}(14)-\mathrm{C}(13)-\mathrm{C}(15)$ & $123.6(9)$ \\
\hline $\mathrm{C}(2)-\mathrm{C}(1)-\operatorname{Ir}(1)$ & $128.8(5)$ & $\mathrm{C}(13) \# 1-\mathrm{C}(14)-\mathrm{C}(13)$ & $138.6(9)$ \\
\hline $\mathrm{C}(3)-\mathrm{C}(2)-\mathrm{C}(1)$ & $121.6(7)$ & & \\
\hline
\end{tabular}

Symmetry transformations used to generate equivalent atoms:

\# $1-x+1 / 2,-y+3 / 2, z$ 
Table S9. Bond lengths $[\AA]$ and angles $\left[^{\circ}\right]$ for $\operatorname{Ir}(\mathbf{p p y S C F})_{2}$ 2(pic).

\begin{tabular}{|c|c|c|c|}
\hline \multicolumn{4}{|c|}{ Bond lengths $[\AA]$} \\
\hline $\operatorname{Ir}(1)-C(1)$ & $1.935(2)$ & $\mathrm{C}(1)-\mathrm{C}(6)$ & $1.410(2)$ \\
\hline $\operatorname{Ir}(1)-C(13)$ & $1.966(2)$ & $C(1)-C(2)$ & $1.441(2)$ \\
\hline $\operatorname{Ir}(1)-N(2)$ & $2.043(1)$ & $C(2)-C(3)$ & $1.372(2)$ \\
\hline $\operatorname{Ir}(1)-\mathrm{N}(1)$ & $2.046(1)$ & $C(3)-C(4)$ & $1.424(2)$ \\
\hline $\operatorname{Ir}(1)-N(3)$ & $2.144(1)$ & $C(4)-C(5)$ & $1.373(2)$ \\
\hline $\operatorname{Ir}(1)-\mathrm{O}(5)$ & $2.144(1)$ & $C(5)-C(6)$ & $1.422(2)$ \\
\hline $\mathrm{S}(1)-\mathrm{O}(2)$ & $1.416(2)$ & $C(6)-C(7)$ & $1.401(2)$ \\
\hline $\mathrm{S}(1)-\mathrm{O}(1)$ & $1.431(2)$ & $C(7)-C(8)$ & $1.472(2)$ \\
\hline $\mathrm{S}(1)-\mathrm{C}(4)$ & $1.719(2)$ & $\mathrm{C}(8)-\mathrm{C}(9)$ & $1.401(3)$ \\
\hline $\mathrm{S}(1)-\mathrm{C}(12)$ & $1.771(3)$ & $\mathrm{C}(9)-\mathrm{C}(10)$ & $1.341(3)$ \\
\hline $\mathrm{S}(2)-\mathrm{O}(4)$ & $1.396(1)$ & $C(10)-C(11)$ & $1.391(2)$ \\
\hline $\mathrm{S}(2)-\mathrm{O}(3)$ & $1.421(1)$ & $C(13)-C(14)$ & $1.403(2)$ \\
\hline$S(2)-C(16)$ & $1.725(2)$ & $\mathrm{C}(13)-\mathrm{C}(18)$ & $1.442(2)$ \\
\hline $\mathrm{S}(2)-\mathrm{C}(24)$ & $1.812(2)$ & $C(14)-C(15)$ & $1.381(2)$ \\
\hline $\mathrm{F}(1)-\mathrm{C}(12)$ & $1.312(4)$ & $C(15)-C(16)$ & $1.421(2)$ \\
\hline $\mathrm{F}(2)-\mathrm{C}(12)$ & $1.284(3)$ & $C(16)-C(17)$ & $1.372(2)$ \\
\hline $\mathrm{F}(3)-\mathrm{C}(12)$ & $1.272(3)$ & $\mathrm{C}(17)-\mathrm{C}(18)$ & $1.342(2)$ \\
\hline $\mathrm{F}(4)-\mathrm{C}(24)$ & $1.312(3)$ & $\mathrm{C}(18)-\mathrm{C}(19)$ & $1.465(2)$ \\
\hline $\mathrm{F}(5)-\mathrm{C}(24)$ & $1.321(3)$ & $C(19)-C(20)$ & $1.412(2)$ \\
\hline $\mathrm{F}(6)-\mathrm{C}(24)$ & $1.291(3)$ & $C(20)-C(21)$ & $1.391(2)$ \\
\hline $\mathrm{O}(5)-\mathrm{C}(30)$ & $1.292(2)$ & $\mathrm{C}(21)-\mathrm{C}(22)$ & $1.391(3)$ \\
\hline $\mathrm{O}(6)-\mathrm{C}(30)$ & $1.171(2)$ & $\mathrm{C}(22)-\mathrm{C}(23)$ & $1.332(2)$ \\
\hline $\mathrm{N}(1)-\mathrm{C}(7)$ & $1.300(2)$ & $C(25)-C(26)$ & $1.352(2)$ \\
\hline $\mathrm{N}(1)-\mathrm{C}(11)$ & $1.354(2)$ & $C(26)-C(27)$ & $1.341(3)$ \\
\hline $\mathrm{N}(2)-\mathrm{C}(23)$ & $1.316(2)$ & $\mathrm{C}(27)-\mathrm{C}(28)$ & $1.322(3)$ \\
\hline $\mathrm{N}(2)-\mathrm{C}(19)$ & $1.392(2)$ & $\mathrm{C}(28)-\mathrm{C}(29)$ & $1.372(3)$ \\
\hline $\mathrm{N}(3)-\mathrm{C}(25)$ & $1.374(2)$ & $C(29)-C(30)$ & $1.534(3)$ \\
\hline $\mathrm{N}(3)-\mathrm{C}(29)$ & $1.373(2)$ & & \\
\hline \multicolumn{4}{|c|}{ Bond angles $\left[{ }^{\circ}\right]$} \\
\hline$C(1)-\operatorname{Ir}(1)-C(13)$ & $90.6(6)$ & $\mathrm{N}(1)-\mathrm{C}(7)-\mathrm{C}(6)$ & $117.8(1)$ \\
\hline $\mathrm{C}(1)-\operatorname{Ir}(1)-\mathrm{N}(2)$ & $97.3(5)$ & $\mathrm{N}(1)-\mathrm{C}(7)-\mathrm{C}(8)$ & $117.6(1)$ \\
\hline $\mathrm{C}(13)-\operatorname{Ir}(1)-\mathrm{N}(2)$ & $81.0(6)$ & $C(6)-C(7)-C(8)$ & $124.4(1)$ \\
\hline $\mathrm{C}(1)-\operatorname{Ir}(1)-\mathrm{N}(1)$ & $79.7(5)$ & $\mathrm{C}(9)-\mathrm{C}(8)-\mathrm{C}(7)$ & $118.1(2)$ \\
\hline $\mathrm{C}(13)-\operatorname{Ir}(1)-\mathrm{N}(1)$ & $95.7(5)$ & $\mathrm{C}(10)-\mathrm{C}(9)-\mathrm{C}(8)$ & $120.6(2)$ \\
\hline $\mathrm{N}(2)-\operatorname{Ir}(1)-\mathrm{N}(1)$ & $175.5(5)$ & $\mathrm{C}(9)-\mathrm{C}(10)-\mathrm{C}(11)$ & $119.5(2)$ \\
\hline $\mathrm{C}(1)-\operatorname{Ir}(1)-\mathrm{N}(3)$ & $100.5(6)$ & $\mathrm{N}(1)-\mathrm{C}(11)-\mathrm{C}(10)$ & $120.4(2)$ \\
\hline$C(13)-\operatorname{Ir}(1)-N(3)$ & $168.2(6)$ & $\mathrm{F}(3)-\mathrm{C}(12)-\mathrm{F}(2)$ & $105.0(3)$ \\
\hline $\mathrm{N}(2)-\operatorname{Ir}(1)-\mathrm{N}(3)$ & $93.5(5)$ & $\mathrm{F}(3)-\mathrm{C}(12)-\mathrm{F}(1)$ & $110.0(3)$ \\
\hline $\mathrm{N}(1)-\operatorname{Ir}(1)-\mathrm{N}(3)$ & $90.3(5)$ & $\mathrm{F}(2)-\mathrm{C}(12)-\mathrm{F}(1)$ & $103.0(3)$ \\
\hline $\mathrm{C}(1)-\operatorname{Ir}(1)-\mathrm{O}(5)$ & $174.8(6)$ & $\mathrm{F}(3)-\mathrm{C}(12)-\mathrm{S}(1)$ & $114.0(3)$ \\
\hline $\mathrm{C}(13)-\operatorname{Ir}(1)-\mathrm{O}(5)$ & $92.5(5)$ & $\mathrm{F}(2)-\mathrm{C}(12)-\mathrm{S}(1)$ & $113.0(3)$ \\
\hline $\mathrm{N}(2)-\operatorname{Ir}(1)-\mathrm{O}(5)$ & $87.3(4)$ & $\mathrm{F}(1)-\mathrm{C}(12)-\mathrm{S}(1)$ & $110.0(2)$ \\
\hline $\mathrm{N}(1)-\operatorname{Ir}(1)-\mathrm{O}(5)$ & $95.8(4)$ & $\mathrm{C}(14)-\mathrm{C}(13)-\mathrm{C}(18)$ & $114.2(2)$ \\
\hline $\mathrm{N}(3)-\operatorname{Ir}(1)-\mathrm{O}(5)$ & $76.8(5)$ & $C(14)-C(13)-\operatorname{Ir}(1)$ & $129.7(1)$ \\
\hline $\mathrm{O}(2)-\mathrm{S}(1)-\mathrm{O}(1)$ & $121.0(1)$ & $C(18)-C(13)-\operatorname{Ir}(1)$ & $116.1(1)$ \\
\hline $\mathrm{O}(2)-\mathrm{S}(1)-\mathrm{C}(4)$ & $110.7(8)$ & $C(15)-C(14)-C(13)$ & $122.8(2)$ \\
\hline $\mathrm{O}(1)-\mathrm{S}(1)-\mathrm{C}(4)$ & $109.1(1)$ & $C(14)-C(15)-C(16)$ & $119.6(2)$ \\
\hline $\mathrm{O}(2)-\mathrm{S}(1)-\mathrm{C}(12)$ & $103.1(1)$ & $C(17)-C(16)-C(15)$ & $118.9(2)$ \\
\hline $\mathrm{O}(1)-\mathrm{S}(1)-\mathrm{C}(12)$ & $106.2(2)$ & $\mathrm{C}(17)-\mathrm{C}(16)-\mathrm{S}(2)$ & $123.2(1)$ \\
\hline $\mathrm{C}(4)-\mathrm{S}(1)-\mathrm{C}(12)$ & $105.4(1)$ & $\mathrm{C}(15)-\mathrm{C}(16)-\mathrm{S}(2)$ & $117.9(1)$ \\
\hline $\mathrm{O}(4)-\mathrm{S}(2)-\mathrm{O}(3)$ & $121.1(9)$ & $\mathrm{C}(18)-\mathrm{C}(17)-\mathrm{C}(16)$ & $120.4(1)$ \\
\hline $\mathrm{O}(4)-\mathrm{S}(2)-\mathrm{C}(16)$ & $110.8(8)$ & $\mathrm{C}(17)-\mathrm{C}(18)-\mathrm{C}(13)$ & $124.0(1)$ \\
\hline $\mathrm{O}(3)-\mathrm{S}(2)-\mathrm{C}(16)$ & $109.2(8)$ & $\mathrm{C}(17)-\mathrm{C}(18)-\mathrm{C}(19)$ & $122.4(1)$ \\
\hline $\mathrm{O}(4)-\mathrm{S}(2)-\mathrm{C}(24)$ & $104.1(1)$ & $\mathrm{C}(13)-\mathrm{C}(18)-\mathrm{C}(19)$ & $113.5(1)$ \\
\hline $\mathrm{O}(3)-\mathrm{S}(2)-\mathrm{C}(24)$ & $104.7(1)$ & $\mathrm{N}(2)-\mathrm{C}(19)-\mathrm{C}(20)$ & $119.0(1)$ \\
\hline
\end{tabular}




\begin{tabular}{cccc}
\hline $\mathrm{C}(16)-\mathrm{S}(2)-\mathrm{C}(24)$ & $105.5(1)$ & $\mathrm{N}(2)-\mathrm{C}(19)-\mathrm{C}(18)$ & $114.0(1)$ \\
$\mathrm{C}(30)-\mathrm{O}(5)-\mathrm{Ir}(1)$ & $117.9(1)$ & $\mathrm{C}(20)-\mathrm{C}(19)-\mathrm{C}(18)$ & $127.0(2)$ \\
$\mathrm{C}(7)-\mathrm{N}(1)-\mathrm{C}(11)$ & $123.4(1)$ & $\mathrm{C}(21)-\mathrm{C}(20)-\mathrm{C}(19)$ & $119.3(2)$ \\
$\mathrm{C}(7)-\mathrm{N}(1)-\operatorname{Ir}(1)$ & $113.7(9)$ & $\mathrm{C}(20)-\mathrm{C}(21)-\mathrm{C}(22)$ & $119.8(2)$ \\
$\mathrm{C}(11)-\mathrm{N}(1)-\operatorname{Ir}(1)$ & $122.6(1)$ & $\mathrm{C}(23)-\mathrm{C}(22)-\mathrm{C}(21)$ & $117.1(2)$ \\
$\mathrm{C}(23)-\mathrm{N}(2)-\mathrm{C}(19)$ & $117.4(1)$ & $\mathrm{N}(2)-\mathrm{C}(23)-\mathrm{C}(22)$ & $127.2(2)$ \\
$\mathrm{C}(23)-\mathrm{N}(2)-\mathrm{Ir}(1)$ & $127.4(1)$ & $\mathrm{F}(6)-\mathrm{C}(24)-\mathrm{F}(4)$ & $107.0(2)$ \\
$\mathrm{C}(19)-\mathrm{N}(2)-\mathrm{Ir}(1)$ & $115.2(9)$ & $\mathrm{F}(6)-\mathrm{C}(24)-\mathrm{F}(5)$ & $108.0(2)$ \\
$\mathrm{C}(25)-\mathrm{N}(3)-\mathrm{C}(29)$ & $117.2(2)$ & $\mathrm{F}(4)-\mathrm{C}(24)-\mathrm{F}(5)$ & $107.0(2)$ \\
$\mathrm{C}(25)-\mathrm{N}(3)-\operatorname{Ir}(1)$ & $128.5(1)$ & $\mathrm{F}(6)-\mathrm{C}(24)-\mathrm{S}(2)$ & $113.0(2)$ \\
$\mathrm{C}(29)-\mathrm{N}(3)-\mathrm{Ir}(1)$ & $113.9(1)$ & $\mathrm{F}(4)-\mathrm{C}(24)-\mathrm{S}(2)$ & $112.5(2)$ \\
$\mathrm{C}(6)-\mathrm{C}(1)-\mathrm{C}(2)$ & $115.1(1)$ & $\mathrm{F}(5)-\mathrm{C}(24)-\mathrm{S}(2)$ & $109.9(2)$ \\
$\mathrm{C}(6)-\mathrm{C}(1)-\mathrm{Ir}(1)$ & $116.1(1)$ & $\mathrm{C}(26)-\mathrm{C}(25)-\mathrm{N}(3)$ & $120.6(2)$ \\
$\mathrm{C}(2)-\mathrm{C}(1)-\operatorname{Ir}(1)$ & $128.8(1)$ & $\mathrm{C}(27)-\mathrm{C}(26)-\mathrm{C}(25)$ & $122.0(2)$ \\
$\mathrm{C}(3)-\mathrm{C}(2)-\mathrm{C}(1)$ & $123.2(2)$ & $\mathrm{C}(28)-\mathrm{C}(27)-\mathrm{C}(26)$ & $118.0(2)$ \\
$\mathrm{C}(2)-\mathrm{C}(3)-\mathrm{C}(4)$ & $118.1(2)$ & $\mathrm{C}(27)-\mathrm{C}(28)-\mathrm{C}(29)$ & $122.5(2)$ \\
$\mathrm{C}(5)-\mathrm{C}(4)-\mathrm{C}(3)$ & $122.8(1)$ & $\mathrm{N}(3)-\mathrm{C}(29)-\mathrm{C}(28)$ & $119.8(2)$ \\
$\mathrm{C}(5)-\mathrm{C}(4)-\mathrm{S}(1)$ & $120.3(1)$ & $\mathrm{N}(3)-\mathrm{C}(29)-\mathrm{C}(30)$ & $115.8(2)$ \\
$\mathrm{C}(3)-\mathrm{C}(4)-\mathrm{S}(1)$ & $116.9(1)$ & $\mathrm{C}(28)-\mathrm{C}(29)-\mathrm{C}(30)$ & $124.3(2)$ \\
$\mathrm{C}(4)-\mathrm{C}(5)-\mathrm{C}(6)$ & $117.1(1)$ & $\mathrm{O}(6)-\mathrm{C}(30)-\mathrm{O}(5)$ & $125.0(2)$ \\
$\mathrm{C}(7)-\mathrm{C}(6)-\mathrm{C}(1)$ & $112.3(1)$ & $\mathrm{O}(6)-\mathrm{C}(30)-\mathrm{C}(29)$ & $120.4(2)$ \\
$\mathrm{C}(7)-\mathrm{C}(6)-\mathrm{C}(5)$ & $123.9(1)$ & $\mathrm{O}(5)-\mathrm{C}(30)-\mathrm{C}(29)$ & $114.5(2)$ \\
$\mathrm{C}(1)-\mathrm{C}(6)-\mathrm{C}(5)$ & $123.7(1)$ & & \\
& & & \\
\hline
\end{tabular}


Table S10. Bond lengths $[\AA]$ and angles $\left[^{\circ}\right]$ for $\operatorname{Ir}(\mathbf{p p y S C F} 3)_{2}$ (bor).

\begin{tabular}{|c|c|c|c|}
\hline \multicolumn{4}{|c|}{ Bond lengths $[\AA]$} \\
\hline $\operatorname{Ir}(1)-C(19)$ & $1.993(6)$ & $\mathrm{N}(8)-\mathrm{C}(31)$ & $1.338(1)$ \\
\hline $\operatorname{Ir}(1)-C(7)$ & $2.009(5)$ & $\mathrm{N}(9)-\mathrm{C}(36)$ & $1.364(9)$ \\
\hline $\operatorname{Ir}(1)-\mathrm{N}(2)$ & $2.045(4)$ & $\mathrm{N}(9)-\mathrm{N}(10)$ & $1.377(9)$ \\
\hline $\operatorname{Ir}(1)-\mathrm{N}(1)$ & $2.049(4)$ & $\mathrm{N}(9)-\mathrm{B}(1)$ & $1.527(8)$ \\
\hline $\operatorname{Ir}(1)-N(3)$ & $2.140(5)$ & $\mathrm{N}(10)-\mathrm{C}(34)$ & $1.353(1)$ \\
\hline $\operatorname{Ir}(1)-\mathrm{N}(5)$ & $2.158(5)$ & $\mathrm{C}(1)-\mathrm{C}(2)$ & $1.376(9)$ \\
\hline $\mathrm{S}(1)-\mathrm{O}(1)$ & $1.465(8)$ & $C(2)-C(3)$ & $1.392(1)$ \\
\hline $\mathrm{S}(1)-\mathrm{O}(2)$ & $1.467(1)$ & $C(3)-C(4)$ & $1.331(1)$ \\
\hline $\mathrm{S}(1)-\mathrm{C}(10)$ & $1.736(6)$ & $\mathrm{C}(4)-\mathrm{C}(5)$ & $1.424(8)$ \\
\hline $\mathrm{S}(1)-\mathrm{C}(12)$ & $1.990(2)$ & $C(5)-C(6)$ & $1.469(8)$ \\
\hline $\mathrm{S}(2)-\mathrm{O}(3)$ & $1.435(7)$ & $\mathrm{C}(6)-\mathrm{C}(11)$ & $1.387(8)$ \\
\hline $\mathrm{S}(2)-\mathrm{O}(4)$ & $1.455(7)$ & $\mathrm{C}(6)-\mathrm{C}(7)$ & $1.410(7)$ \\
\hline $\mathrm{S}(2)-\mathrm{C}(24)$ & $1.721(2)$ & $C(7)-C(8)$ & $1.396(8)$ \\
\hline $\mathrm{S}(2)-\mathrm{C}(22)$ & $1.766(7)$ & $\mathrm{C}(8)-\mathrm{C}(9)$ & $1.382(9)$ \\
\hline $\mathrm{F}(1)-\mathrm{C}(12)$ & $1.150(3)$ & $\mathrm{C}(9)-\mathrm{C}(10)$ & $1.387(1)$ \\
\hline $\mathrm{F}(2)-\mathrm{C}(12)$ & $1.300(2)$ & $C(10)-C(11)$ & $1.393(9)$ \\
\hline $\mathrm{F}(3)-\mathrm{C}(12)$ & $1.437(2)$ & $C(13)-C(14)$ & $1.382(9)$ \\
\hline $\mathrm{F}(4)-\mathrm{C}(24)$ & $1.244(2)$ & $\mathrm{C}(14)-\mathrm{C}(15)$ & $1.386(1)$ \\
\hline $\mathrm{F}(5)-\mathrm{C}(24)$ & $1.417(2)$ & $C(15)-C(16)$ & $1.379(1)$ \\
\hline $\mathrm{F}(6)-\mathrm{C}(24)$ & $1.501(2)$ & $C(16)-C(17)$ & $1.399(8)$ \\
\hline $\mathrm{N}(1)-\mathrm{C}(1)$ & $1.345(8)$ & $\mathrm{C}(17)-\mathrm{C}(18)$ & $1.482(9)$ \\
\hline $\mathrm{N}(1)-\mathrm{C}(5)$ & $1.359(7)$ & $\mathrm{C}(18)-\mathrm{C}(23)$ & $1.391(1)$ \\
\hline $\mathrm{N}(2)-\mathrm{C}(17)$ & $1.355(9)$ & $\mathrm{C}(18)-\mathrm{C}(19)$ & $1.425(7)$ \\
\hline $\mathrm{N}(2)-\mathrm{C}(13)$ & $1.366(8)$ & $C(19)-C(20)$ & $1.431(9)$ \\
\hline $\mathrm{N}(3)-\mathrm{C}(27)$ & $1.344(9)$ & $\mathrm{C}(20)-\mathrm{C}(21)$ & $1.353(1)$ \\
\hline $\mathrm{N}(3)-\mathrm{N}(4)$ & $1.357(7)$ & $\mathrm{C}(21)-\mathrm{C}(22)$ & $1.400(9)$ \\
\hline $\mathrm{N}(4)-\mathrm{C}(25)$ & $1.366(8)$ & $\mathrm{C}(22)-\mathrm{C}(23)$ & $1.368(1)$ \\
\hline $\mathrm{N}(4)-\mathrm{B}(1)$ & $1.534(9)$ & $C(25)-C(26)$ & $1.312(1)$ \\
\hline $\mathrm{N}(5)-\mathrm{C}(30)$ & $1.315(8)$ & $C(26)-C(27)$ & $1.410(1)$ \\
\hline $\mathrm{N}(5)-\mathrm{N}(6)$ & $1.356(6)$ & $\mathrm{C}(28)-\mathrm{C}(29)$ & $1.366(1)$ \\
\hline $\mathrm{N}(6)-\mathrm{C}(28)$ & $1.358(9)$ & $C(29)-C(30)$ & $1.386(1)$ \\
\hline $\mathrm{N}(6)-\mathrm{B}(1)$ & $1.550(8)$ & $C(31)-C(32)$ & $1.294(2)$ \\
\hline N(7)-N(8) & $1.330(9)$ & $C(32)-C(33)$ & $1.396(1)$ \\
\hline $\mathrm{N}(7)-\mathrm{C}(33)$ & $1.356(9)$ & $\mathrm{C}(34)-\mathrm{C}(35)$ & $1.367(2)$ \\
\hline $\mathrm{N}(7)-\mathrm{B}(1)$ & $1.552(8)$ & $\mathrm{C}(35)-\mathrm{C}(36)$ & $1.369(1)$ \\
\hline \multicolumn{4}{|c|}{ Bond angles $\left[{ }^{\circ}\right]$} \\
\hline$C(19)-\operatorname{Ir}(1)-C(7)$ & $86.7(2)$ & $\mathrm{C}(11)-\mathrm{C}(6)-\mathrm{C}(5)$ & $123.0(5)$ \\
\hline$C(19)-\operatorname{Ir}(1)-N(2)$ & $80.4(2)$ & $C(7)-C(6)-C(5)$ & $114.4(5)$ \\
\hline $\mathrm{C}(7)-\operatorname{Ir}(1)-\mathrm{N}(2)$ & $95.5(2)$ & $C(8)-C(7)-C(6)$ & $116.9(5)$ \\
\hline $\mathrm{C}(19)-\operatorname{Ir}(1)-\mathrm{N}(1)$ & $92.6(2)$ & $C(8)-C(7)-\operatorname{Ir}(1)$ & $128.0(4)$ \\
\hline$C(7)-\operatorname{Ir}(1)-N(1)$ & $80.2(2)$ & $C(6)-C(7)-\operatorname{Ir}(1)$ & $115.1(4)$ \\
\hline $\mathrm{N}(2)-\operatorname{Ir}(1)-\mathrm{N}(1)$ & $172.0(2)$ & $\mathrm{C}(9)-\mathrm{C}(8)-\mathrm{C}(7)$ & $122.1(6)$ \\
\hline $\mathrm{C}(19)-\operatorname{Ir}(1)-\mathrm{N}(3)$ & $94.6(2)$ & $\mathrm{C}(8)-\mathrm{C}(9)-\mathrm{C}(10)$ & $119.0(6)$ \\
\hline $\mathrm{C}(7)-\operatorname{Ir}(1)-\mathrm{N}(3)$ & $176.3(2)$ & $C(9)-C(10)-C(11)$ & $121.7(6)$ \\
\hline $\mathrm{N}(2)-\operatorname{Ir}(1)-\mathrm{N}(3)$ & $88.1(2)$ & $\mathrm{C}(9)-\mathrm{C}(10)-\mathrm{S}(1)$ & $119.5(5)$ \\
\hline $\mathrm{N}(1)-\operatorname{Ir}(1)-\mathrm{N}(3)$ & $96.3(2)$ & $\mathrm{C}(11)-\mathrm{C}(10)-\mathrm{S}(1)$ & $118.8(5)$ \\
\hline$C(19)-\operatorname{Ir}(1)-N(5)$ & $172.8(2)$ & $C(6)-C(11)-C(10)$ & $117.7(5)$ \\
\hline$C(7)-\operatorname{Ir}(1)-N(5)$ & $91.4(2)$ & $\mathrm{F}(1)-\mathrm{C}(12)-\mathrm{F}(2)$ & $125.0(3)$ \\
\hline $\mathrm{N}(2)-\operatorname{Ir}(1)-\mathrm{N}(5)$ & $92.9(2)$ & $\mathrm{F}(1)-\mathrm{C}(12)-\mathrm{F}(3)$ & $108.4(1)$ \\
\hline $\mathrm{N}(1)-\operatorname{Ir}(1)-\mathrm{N}(5)$ & $93.9(2)$ & $\mathrm{F}(2)-\mathrm{C}(12)-\mathrm{F}(3)$ & $107.5(2)$ \\
\hline $\mathrm{N}(3)-\operatorname{Ir}(1)-\mathrm{N}(5)$ & $87.8(2)$ & $\mathrm{F}(1)-\mathrm{C}(12)-\mathrm{S}(1)$ & $109.8(1)$ \\
\hline $\mathrm{O}(1)-\mathrm{S}(1)-\mathrm{O}(2)$ & $122.4(7)$ & $\mathrm{F}(2)-\mathrm{C}(12)-\mathrm{S}(1)$ & $106.5(2)$ \\
\hline $\mathrm{O}(1)-\mathrm{S}(1)-\mathrm{C}(10)$ & $109.6(4)$ & $\mathrm{F}(3)-\mathrm{C}(12)-\mathrm{S}(1)$ & $95.5(2)$ \\
\hline $\mathrm{O}(2)-\mathrm{S}(1)-\mathrm{C}(10)$ & $110.1(4)$ & $\mathrm{N}(2)-\mathrm{C}(13)-\mathrm{C}(14)$ & $122.3(7)$ \\
\hline $\mathrm{O}(1)-\mathrm{S}(1)-\mathrm{C}(12)$ & $102.6(7)$ & $C(13)-C(14)-C(15)$ & $119.1(7)$ \\
\hline
\end{tabular}




\begin{tabular}{|c|c|c|c|}
\hline $\mathrm{O}(2)-\mathrm{S}(1)-\mathrm{C}(12)$ & $110.5(9)$ & $C(16)-C(15)-C(14)$ & $119.2(6)$ \\
\hline$C(10)-S(1)-C(12)$ & $98.9(6)$ & $\mathrm{C}(15)-\mathrm{C}(16)-\mathrm{C}(17)$ & $119.8(7)$ \\
\hline $\mathrm{O}(3)-\mathrm{S}(2)-\mathrm{O}(4)$ & $120.9(5)$ & $\mathrm{N}(2)-\mathrm{C}(17)-\mathrm{C}(16)$ & $121.2(6)$ \\
\hline $\mathrm{O}(3)-\mathrm{S}(2)-\mathrm{C}(24)$ & 107.2(7) & $\mathrm{N}(2)-\mathrm{C}(17)-\mathrm{C}(18)$ & $113.3(5)$ \\
\hline $\mathrm{O}(4)-\mathrm{S}(2)-\mathrm{C}(24)$ & $105.9(7)$ & $\mathrm{C}(16)-\mathrm{C}(17)-\mathrm{C}(18)$ & $125.5(6)$ \\
\hline $\mathrm{O}(3)-\mathrm{S}(2)-\mathrm{C}(22)$ & 109.1(4) & $\mathrm{C}(23)-\mathrm{C}(18)-\mathrm{C}(19)$ & $122.3(6)$ \\
\hline $\mathrm{O}(4)-\mathrm{S}(2)-\mathrm{C}(22)$ & $110.8(4)$ & $\mathrm{C}(23)-\mathrm{C}(18)-\mathrm{C}(17)$ & $123.4(5)$ \\
\hline $\mathrm{C}(24)-\mathrm{S}(2)-\mathrm{C}(22)$ & $100.7(6)$ & $\mathrm{C}(19)-\mathrm{C}(18)-\mathrm{C}(17)$ & $114.3(6)$ \\
\hline $\mathrm{C}(1)-\mathrm{N}(1)-\mathrm{C}(5)$ & $119.2(5)$ & $\mathrm{C}(18)-\mathrm{C}(19)-\mathrm{C}(20)$ & $115.7(6)$ \\
\hline $\mathrm{C}(1)-\mathrm{N}(1)-\operatorname{Ir}(1)$ & $124.2(4)$ & $\mathrm{C}(18)-\mathrm{C}(19)-\operatorname{Ir}(1)$ & $114.9(5)$ \\
\hline $\mathrm{C}(5)-\mathrm{N}(1)-\operatorname{Ir}(1)$ & $115.8(3)$ & $C(20)-C(19)-\operatorname{Ir}(1)$ & $129.2(4)$ \\
\hline $\mathrm{C}(17)-\mathrm{N}(2)-\mathrm{C}(13)$ & $118.4(5)$ & $\mathrm{C}(21)-\mathrm{C}(20)-\mathrm{C}(19)$ & $122.2(5)$ \\
\hline $\mathrm{C}(17)-\mathrm{N}(2)-\operatorname{Ir}(1)$ & $116.7(4)$ & $\mathrm{C}(20)-\mathrm{C}(21)-\mathrm{C}(22)$ & $119.3(7)$ \\
\hline $\mathrm{C}(13)-\mathrm{N}(2)-\operatorname{Ir}(1)$ & $124.9(4)$ & $\mathrm{C}(23)-\mathrm{C}(22)-\mathrm{C}(21)$ & $122.3(6)$ \\
\hline $\mathrm{C}(27)-\mathrm{N}(3)-\mathrm{N}(4)$ & 107.1(5) & $\mathrm{C}(23)-\mathrm{C}(22)-\mathrm{S}(2)$ & $118.9(5)$ \\
\hline $\mathrm{C}(27)-\mathrm{N}(3)-\operatorname{Ir}(1)$ & $125.9(4)$ & $\mathrm{C}(21)-\mathrm{C}(22)-\mathrm{S}(2)$ & $118.8(6)$ \\
\hline $\mathrm{N}(4)-\mathrm{N}(3)-\operatorname{Ir}(1)$ & $127.0(4)$ & $\mathrm{C}(22)-\mathrm{C}(23)-\mathrm{C}(18)$ & $118.2(5)$ \\
\hline $\mathrm{N}(3)-\mathrm{N}(4)-\mathrm{C}(25)$ & $108.2(6)$ & $\mathrm{F}(4)-\mathrm{C}(24)-\mathrm{F}(5)$ & $110.9(1)$ \\
\hline $\mathrm{N}(3)-\mathrm{N}(4)-\mathrm{B}(1)$ & $124.8(4)$ & $\mathrm{F}(4)-\mathrm{C}(24)-\mathrm{F}(6)$ & 101.2(2) \\
\hline $\mathrm{C}(25)-\mathrm{N}(4)-\mathrm{B}(1)$ & $126.7(5)$ & $\mathrm{F}(5)-\mathrm{C}(24)-\mathrm{F}(6)$ & $105.4(1)$ \\
\hline $\mathrm{C}(30)-\mathrm{N}(5)-\mathrm{N}(6)$ & 106.3(5) & $\mathrm{F}(4)-\mathrm{C}(24)-\mathrm{S}(2)$ & $119.2(1)$ \\
\hline $\mathrm{C}(30)-\mathrm{N}(5)-\operatorname{Ir}(1)$ & $126.3(4)$ & $\mathrm{F}(5)-\mathrm{C}(24)-\mathrm{S}(2)$ & $109.5(1)$ \\
\hline $\mathrm{N}(6)-\mathrm{N}(5)-\operatorname{Ir}(1)$ & 126.1(4) & $\mathrm{F}(6)-\mathrm{C}(24)-\mathrm{S}(2)$ & 109.4(9) \\
\hline $\mathrm{N}(5)-\mathrm{N}(6)-\mathrm{C}(28)$ & $109.7(5)$ & $\mathrm{C}(26)-\mathrm{C}(25)-\mathrm{N}(4)$ & $109.8(6)$ \\
\hline $\mathrm{N}(5)-\mathrm{N}(6)-\mathrm{B}(1)$ & $122.5(5)$ & $\mathrm{C}(25)-\mathrm{C}(26)-\mathrm{C}(27)$ & $106.4(7)$ \\
\hline $\mathrm{C}(28)-\mathrm{N}(6)-\mathrm{B}(1)$ & $126.2(5)$ & $\mathrm{N}(3)-\mathrm{C}(27)-\mathrm{C}(26)$ & $108.5(7)$ \\
\hline $\mathrm{N}(8)-\mathrm{N}(7)-\mathrm{C}(33)$ & $111.0(6)$ & $\mathrm{N}(6)-\mathrm{C}(28)-\mathrm{C}(29)$ & $107.7(6)$ \\
\hline $\mathrm{N}(8)-\mathrm{N}(7)-\mathrm{B}(1)$ & $121.5(5)$ & $\mathrm{C}(28)-\mathrm{C}(29)-\mathrm{C}(30)$ & $105.0(7)$ \\
\hline $\mathrm{C}(33)-\mathrm{N}(7)-\mathrm{B}(1)$ & $127.5(6)$ & $\mathrm{N}(5)-\mathrm{C}(30)-\mathrm{C}(29)$ & $111.3(6)$ \\
\hline $\mathrm{N}(7)-\mathrm{N}(8)-\mathrm{C}(31)$ & 103.6(7) & $\mathrm{C}(32)-\mathrm{C}(31)-\mathrm{N}(8)$ & $114.7(8)$ \\
\hline $\mathrm{C}(36)-\mathrm{N}(9)-\mathrm{N}(10)$ & $112.0(6)$ & $\mathrm{C}(31)-\mathrm{C}(32)-\mathrm{C}(33)$ & $104.8(7)$ \\
\hline $\mathrm{C}(36)-\mathrm{N}(9)-\mathrm{B}(1)$ & $127.6(6)$ & $\mathrm{N}(7)-\mathrm{C}(33)-\mathrm{C}(32)$ & $105.9(8)$ \\
\hline $\mathrm{N}(10)-\mathrm{N}(9)-\mathrm{B}(1)$ & $119.4(6)$ & $\mathrm{N}(10)-\mathrm{C}(34)-\mathrm{C}(35$ & $112.2(7)$ \\
\hline $\mathrm{C}(34)-\mathrm{N}(10)-\mathrm{N}(9)$ & $103.1(7)$ & $\mathrm{C}(34)-\mathrm{C}(35)-\mathrm{C}(36)$ & $106.7(7)$ \\
\hline $\mathrm{N}(1)-\mathrm{C}(1)-\mathrm{C}(2)$ & $122.3(6)$ & $\mathrm{N}(9)-\mathrm{C}(36)-\mathrm{C}(35)$ & 106.1(8) \\
\hline $\mathrm{C}(1)-\mathrm{C}(2)-\mathrm{C}(3)$ & $118.5(6)$ & $\mathrm{N}(9)-\mathrm{B}(1)-\mathrm{N}(4)$ & $111.8(6)$ \\
\hline $\mathrm{C}(4)-\mathrm{C}(3)-\mathrm{C}(2)$ & $120.2(6)$ & $\mathrm{N}(9)-\mathrm{B}(1)-\mathrm{N}(6)$ & $106.9(5)$ \\
\hline $\mathrm{C}(3)-\mathrm{C}(4)-\mathrm{C}(5)$ & $120.1(6)$ & $\mathrm{N}(4)-\mathrm{B}(1)-\mathrm{N}(6)$ & $113.0(4)$ \\
\hline $\mathrm{N}(1)-\mathrm{C}(5)-\mathrm{C}(4)$ & $119.5(5)$ & $\mathrm{N}(9)-\mathrm{B}(1)-\mathrm{N}(7)$ & 109.6(4) \\
\hline $\mathrm{N}(1)-\mathrm{C}(5)-\mathrm{C}(6)$ & $114.4(4)$ & $\mathrm{N}(4)-\mathrm{B}(1)-\mathrm{N}(7)$ & $107.3(5)$ \\
\hline $\mathrm{C}(4)-\mathrm{C}(5)-\mathrm{C}(6)$ & $126.0(5)$ & $\mathrm{N}(6)-\mathrm{B}(1)-\mathrm{N}(7)$ & 108.1(5) \\
\hline $\mathrm{C}(11)-\mathrm{C}(6)-\mathrm{C}(7)$ & $122.6(5)$ & & \\
\hline
\end{tabular}




\section{References}

(S1) Kim, J.-H.; Kim, S.-Y.; Cho, Y.-J.; Son, H.-J.; Cho, D. W.; Kang, S. O. A detailed evaluation for the nonradiative processes in highly phosphorescent Iridium(III) complexes. J. Phys. Chem. C 2018, 122, 4029-4036.

(S2) Hoang, T. N. Y.; Humbert-Droz, M.; Dutronc, T.; Guénée, L.; Besnard, C.; Piguet, C.; A polyaromatic terdentate binding unit with fused 5,6-membered chelates for complexing s-, p-, d-, and f-block cations. Inorg. Chem. 2013, 52, 5570-5580.

(S3) Frisch, M. J.; Trucks, G. W.; Schlegel, H. B.; Scuseria, G. E.; Robb, M. A.; Cheeseman, J. R.; Scalmani, G.; Barone, V.; Mennucci, B.; Petersson, G. A.; Nakatsuji, H.; Caricato, M.; Li, X.; Hratchian, H. P.; Izmaylov, A. F.; Bloino, J.; Zheng, G.; Sonnenberg, J. L.; Hada, M.; Ehara, M.; Toyota, K.; Fukuda, R.; Hasegawa, J.; Ishida, M.; Nakajima, T.; Honda, Y.; Kitao, O.; Nakai, H.; Vreven, T.; Montgomery Jr., J. A.; Peralta, J. E.; Ogliaro, F.; Bearpark, M.; Heyd, J. J.; Brothers, E.; Kudin, K. N.; Staroverov, V. N.; Kobayashi, R.; Normand, J.; Raghavachari, K.; Rendell, A.; Burant, J. C.; Iyengar, S. S.; Tomasi, J.; Cossi, M.; Rega, N.; Millam, J. M.; Klene, M.; Knox, J. E.; Cross, J. B.; Bakken, V.; Adamo, C.; Jaramillo, J.; Gomperts, R.; Stratmann, R. E.; Yazyev, O.; Austin, A. J.; Cammi, R.; Pomelli, C.; Ochterski, J. W.; Martin, R. L.; Morokuma, K.; Zakrzewski, V. G.; Voth, G. A.; Salvador, P.; Dannenberg, J. J.; Dapprich, S.; Daniels, A. D.; Farkas, Ö.; Foresman, J. B.; Ortiz, J. V.; Cioslowski J.; Fox, D. J. Gaussian 09, Revision D. 01, Gaussian, Inc., Wallingford CT, 2009.

(S4) Hay, P. J.; Wadt, W. R. Ab initio effective core potentials for molecular calculations. Potentials for $\mathrm{K}$ to Au including the outermost core orbitals. J. Chem. Phys. 1985, 82, 299-310.

(S5) Lee, C.; Yang, W.; Parr, R. G. Development of the colle-salvetti correlationenergy formula into a functional of the electron density. Phys. Rev. B Condens. Matter Mater. Phys. 1988, 37, 785-789.

(S6) Becke, A. D. Density-functional thermochemistry. III. The role of exact exchange. J. Chem. Phys. 1993, 98, 5648-5652.

(S7) SMART and SAINT, Bruker Analytical X-Ray Division, Madison, WI, 2002.

(S8) Sheldrick, G. M. SHELXTL-PLUS Software Package, Bruker Analytical X-Ray Division, Madison, WI, 2002.

(S9) Farrugia, L. J. ORTEP-3 for Windows - a version of ORTEP-III with a graphical user interface (GUI). J. Appl. Crystallogr. 1997, 30, 565-565. 